\title{
Relações entre Subespaços, Ciclicidade e Hiperciclicidade em Espaços de Banach
}

\author{
André Quintal Augusto
}

TESE APRESENTADA

$\mathrm{AO}$

Instituto de Matemática e Estatística

DA

Universidade DE SÃo PAUlo

PARA

OBTENÇÃO DO TÍTULO

$\mathrm{DE}$

DOUTOR EM CIÊNCIAS

\author{
Programa: Matemática \\ Orientador: Prof. Dr. Leonardo Pellegrini Rodrigues \\ Coorientador: Prof. Dr. André Arbex Hallack
}

Durante o desenvolvimento deste trabalho o autor recebeu auxílio financeiro da CAPES e do CNPq.

São Paulo, novembro de 2019. 



\section{Relações entre Subespaços, Ciclicidade e Hiperciclicidade em Espaços de Banach}

Esta versão da dissertação contém as correções e alterações sugeridas pela Comissão Julgadora durante a defesa da versão original do trabalho, realizada em 29/11/2019. Uma cópia da versão original está disponível no Instituto de Matemática e Estatística da Universidade de São Paulo.

Comissão Julgadora:

- Prof. Dr. Leonardo Pellegrini Rodrigues (orientador) - IME-USP

- Prof. Dr. Rogério Augusto dos Santos Fajardo - IME-USP

- Prof. Dr. Leandro Fiorini Aurichi - ICMC-USP

- Prof. Dr. Leandro Candido Batista - UNIFESP

- Prof. Dr. Thiago Grando - UNICENTRO 

Ao Dodô e ao Bimbo. 



\section{Agradecimentos}

Em primeiro lugar, agradeço ao professor Leonardo Pellegrini por ter aceito, mais uma vez, ser meu orientador. Mesmo quando escolhi, ainda no mestrado, um tema não muito conhecido, ele não mediu esforços para estudá-lo e me orientar.

Agradeço também ao meu coorientador, professor André Hallack. Seu conhecimento sobre o tema e valiosas sugestões foram essenciais para que eu pudesse refletir e prosseguir desenvolvendo a tese.

Agradeço aos meus pais, Mirian e Nilton, por tudo que se possa imaginar. O apoio, carinho e principalmente a paciência de ambos foram primordiais para que eu pudesse chegar até aqui. Eu não poderia pedir por pais melhores.

Agradeço a todos os meus amigos que me acompanharam nestes últimos quatro anos. Em particular, quero agradecer ao meu xará André (Tchongo) e ao Matheus (Mulambo) pela amizade de mais de dez anos. Ambos são os irmãos que eu nunca tive. Quero também agradecer ao Anderson, ao Jardes e ao João pelas inúmeras noites de jogatina, importantíssimas para que eu pudesse espairecer depois de longos dias trabalhando nesta tese, e a Carol Freixo pela sua energia contagiante e amizade de tantos anos.

Agradeço à minha família. Em especial, gostaria de agradecer à minha avó Gé. Sua serenidade, generosidade e tantas outras qualidades me inspiram a ser uma pessoa melhor. Tenho muito orgulho de ser seu neto.

Por fim, mas não menos importante, agradeço à Bárbara Campelo. Não há palavras que façam jus ao quanto eu sou grato por estar ao seu lado nos últimos onze anos. Seu amor e seu companheirismo são tão vitais em minha vida quanto o ar. Uma vez que o sentimento é o mesmo, vou tomar a liberdade de repetir aqui o que eu escrevi quatro anos atrás: é impossível imaginar o amanhã sem você. 


\section{Resumo}

\section{AUGuSTO, A. Q. Relações entre Subespaços, Ciclicidade e Hiperciclicidade em Espaços}

de Banach. 2019. 102 f. Tese (Doutorado) - Instituto de Matemática e Estatística, Universidade de São Paulo, São Paulo, 2019.

Dado um espaço de Banach $X$, um operador linear limitado $T$ em $X$ é dito hipercíclico se existir um vetor $x \in X$ tal que o conjunto orb $(x, T):=\left\{x, T x, T^{2} x, T^{3} x, \ldots T^{n} x \ldots\right\}$ é denso em $X$. Em [18], Madore e Martínez-Avendaño estenderam o conceito de hiperciclicidade para subespaços: dado um subespaço $M \subsetneq X$, um operador $T$ é dito sub-hipercíclico em $M$ se existir $x \in X$ tal que orb $(x, T) \cap M$ seja denso em $M$. Sendo um conceito razoavelmente novo, ainda há muita dúvida sobre quais resultados envolvendo operadores hipercíclicos se estendem naturalmente para operadores sub-hipercíclicos. Este trabalho contribui nesse sentido. Entre os resultados obtidos no segundo capítulo, destacamos a existência de operadores sub-hipercíclicos para qualquer subespaço $M$ de um espaço de Banach e a densidade (na topologia da convergência pontual) do conjunto dos operadores sub-hipercíclicos em $\mathcal{B}(X)$. Estudamos ainda no terceiro capítulo o Critério de Sub-Hiperciclicidade, exibindo um contra-exemplo e um novo critério que funciona em espaços de Banach não necessariamente separáveis.

Além disso, no quarto capítulo deste trabalho estudamos também a relação entre hiperciclicidade e ciclicidade via operadores da forma $I+K$, com o intuito de responder a pergunta: será que existe um espaço de Banach onde todo operador hipercíclico satisfaz o chamado Critério de Hiperciclicidade? Por fim, inspirados na relação entre hiperciclicidade e sub-hiperciclicidade, terminamos o trabalho definindo o conceito de sub-ciclicidade e explorando relações entre todos os conceitos vistos na tese.

Palavras-chave: hiperciclicidade, ciclicidade, sub-hiperciclicidade, operadores sub-hipercíclicos. 


\section{Abstract}

AUGUstO, A. Q. Relationships between Subspaces, Ciclicity and Hypercyclicity in

Banach Spaces. 2019. 102 pp. Ph.D. dissertation - Institute of Mathematics and Statistics, University of São Paulo, São Paulo, 2019.

Given a Banach space $X$, a bounded linear operator $T$ in $X$ is hypercylic if, for some $x \in X$, the set $\operatorname{orb}(x, T):=\left\{x, T x, T^{2} x, T^{3} x, \ldots T^{n} x \ldots\right\}$ is dense in $X$. In [18], Madore and MartínezAvendaño extended the notion of hypercyclicity to subspaces: an operator $T$ is subspace-hypercyclic for some subspace $M \subsetneq X$ if there is some $x \in X$ such that $\operatorname{orb}(x, T) \cap M$ is dense in $M$. Since this is a relatively new concept, there is a lot of questions regarding which results for hypercyclic operators holds for subspace-hypercyclic operators. This work contributes in this area. Amongst the results obtained in the second chapter, we highlight the existence of subspace-hypercyclic operators for any given subspace $M$ of a Banach space and the SOT-density of the set of subspace-hypercyclic operators on $\mathcal{B}(X)$. In the third chapter, we study the Subspace-Hypercyclicity Criterion, showing a counter-example to this criterion and devising a new one that works on nonseparable Banach spaces.

Beyond that, on the fourth chapter we also study the relationship between hypercyclicity and cyclicity using scalar-plus-compact operators, with the goal of answering the question: is there a Banach space where every hypercylic operator satisfy the so-called Hypercyclicity Criterion? Lastly, inspired by the relationship between hypercyclicity and subspace-hypercyclicity, we end this work by introducing the concept of subspace-cyclicity and connecting all the concepts studied in this thesis.

Keywords: hypercyclicity, ciclicity, subspace-hypercyclicity, subspace-hypercyclic operators. 


\section{Conteúdo}

Notação $\quad$ ix

Introdução

$\begin{array}{lll}1 & \text { Preliminares } & 1\end{array}$

1.1 Espaços de Banach . . . . . . . . . . . . . . . . . . . . . 1

1.2 Hiperciclicidade . . . . . . . . . . . . . . . . . . . . . . . . . . 4

1.2 .1 O Critério de Hiperciclicidade $\ldots \ldots \ldots \ldots \ldots \ldots$

1.3 Existência de Operadores Hipercíclicos . . . . . . . . . . . . . . . . . . . . . . 9

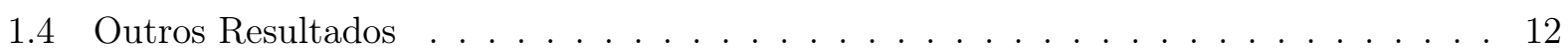

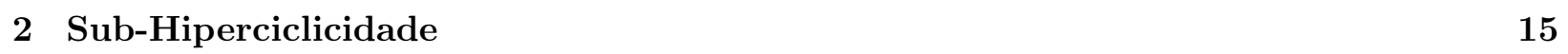

2.1 Definição, Exemplos e Resultados Gerais . . . . . . . . . . . . . . . . . . . . . . . 15

2.2 Operadores Hipercíclicos, Subespaços e Sub-Hiperciclicidade . . . . . . . . . . . . . . 20

2.3 Relações entre Hiperciclicidade e Sub-Hiperciclicidade via Quasi-Conjugações . . . . 26

2.4 Existência de Operadores Sub-Hipercíclicos . . . . . . . . . . . . . . . . 30

$2.5 \quad$ Densidade de Operadores Sub-Hipercíclicos em $\mathcal{B}(X)] \ldots \ldots$. . . . . . . . . 33

\begin{tabular}{|lll}
\hline 3 & O Critério de Sub-Hiperciclicidade & 37
\end{tabular}

3.1 Sub-Transitividade e o Critério de Sub-Hiperciclicidade . . . . . . . . . . . . . . 37

3.2 Um Outro Critério . . . . . . . . . . . . . . . . . . . . . . . . . 44

3.3 Quasi-Conjugações . . . . . . . . . . . . . . . . . . . . . . . . . . . . . . . . . . . . 49

\begin{tabular}{|lll}
\hline & Ciclicidade e Hiperciclicidade & 51
\end{tabular}

4.1 Critério de Ciclicidade . . . . . . . . . . . . . . . . . . . . . . . . . . . . . . 53

4.2 Operadores Hipercíclicos da Forma $\lambda I+S \ldots \ldots \ldots \ldots$

4.2 .1 O Caso em que $S$ é Compacto . . . . . . . . . . . . . . . . . 63

4.3 O Contraexemplo de Bayart e Matheron no Espaço de Argyros-Haydon. . . . . . . . 63

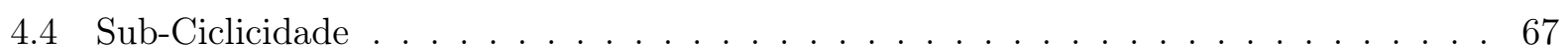

\begin{tabular}{|lll}
5 & Considerações Finais & 77
\end{tabular}

\begin{tabular}{lr}
\hline Bibliografia & 81
\end{tabular} 


\section{Notação}

$\mathbb{N}$ O conjunto $\{1,2,3,4 \ldots\}$.

$\mathbb{N}_{0} \quad$ O conjunto $\{0,1,2,3,4 \ldots\}$.

$\mathbb{K}$ O corpo $\mathbb{R}$ ou $\mathbb{C}$.

$\mathbb{K}[x] \quad \mathrm{O}$ anel formado por todos os polinômios com coeficientes em $\mathbb{K}$ e indeterminada $x$.

span $S \quad$ O conjunto de todas as combinações lineares finitas formadas por elementos de $S$.

$\operatorname{deg} p \quad$ O grau do polinômio $p$.

$B(z, \varepsilon)$ O conjunto $\{x \in X: d(x, z)<\varepsilon\}$.

$\operatorname{orb}(x, T) \quad$ O conjunto $\left\{x, T(x), T^{2}(x), T^{3}(x), \ldots T^{n}(x) \ldots\right\}$

$H(T) \quad$ O conjunto dos vetores hipercíclicos de $T$.

$\operatorname{ker}^{*}(T) \quad$ O conjunto $\cup_{m=1}^{\infty} \operatorname{ker}\left(T^{m}\right)$

$S(T) \quad$ O conjunto $\left\{A^{-1} \circ T \circ A ; A: X \rightarrow X\right.$ linear invertível $\}$

$X^{*}$ O dual topológico do espaço $X$.

$\mathcal{L}(X) \quad \mathrm{O}$ conjunto de todos os operadores lineares em $X$.

$\mathcal{B}(X) \quad \mathrm{O}$ conjunto de todos os operadores lineares contínuos em $X$.

$T^{*} \quad$ O operador adjunto de $T \in \mathcal{B}(X)$.

$\delta_{i j} \quad \mathrm{O}$ conhecido delta de Kronecker. Isto é, $\delta_{i j}=1$ se $i=j$ e $\delta_{i j}=0$ se $i \neq j$.

I O operador identidade no espaço de Banach em questão.

$\bar{M} \quad$ O fecho do conjunto $M$.

$e_{j}$ O vetor canônico $(0,0,0, \ldots 0, \underbrace{1}_{\text {posição } j}, 0, \ldots)$ de $\ell_{p}$ e $c_{0}$.

$c_{00}$ O subconjunto $\operatorname{span}\left\{e_{i}: i \in \mathbb{N}\right\}$ de $\ell_{p}$ e $c_{0}$. 


\section{Introdução}

Seja $X$ um espaço de Banach. Um operador linear limitado $T: X \rightarrow X$ é dito hipercíclico se existir um vetor $x \in X$ tal que o conjunto orb $(x, T):=\left\{x, T x, T^{2} x, T^{3} x, \ldots T^{n} x \ldots\right\}$ é denso em $X$. O vetor $x$ é dito vetor hipercíclico para $T$. Dizemos que $T$ é sub-hipercíclico se existe um $x \in X$ e um subespaço fechado de dimensão infinita não-trivia| ${ }^{1} M$ tal que orb $(x, T) \cap M$ é denso em $M$. Neste caso, dizemos que $T$ é $M$-hipercíclico ou sub-hipercíclico para $M$ e que $x$ é um vetor M-hipercíclico para $T$.

Apesar do primeiro exemplo de operador hipercíclico ter sido dado em 1929 por G.D. Birkhoff (ainda que sem esta nomenclatura), podemos dizer que foi apenas em 1991, a partir do trabalho de Godefroy e Shapiro em [12], que a pesquisa nesta área realmente começou a se desenvolver. Entre os vários resultados obtidos desde então, podemos destacar o Teorema de Ansari (toda potência de um operador hipercíclico é hipercíclico), o Teorema de Ansari-Bernal (todo espaço de Banach separável e de dimensão infinita contém um operador hipercíclico), o Teorema de Herrero-Bourdon (todo operador hipercíclico admite um subespaço denso consistindo, com exceção do vetor nulo, de vetores hipercíclicos) e o importantíssimo Critério de Hiperciclicidade.

O Critério de Hiperciclicidade (ou apenas Critério) é considerado o resultado mais importante na teoria de operadores hipercíclicos. Como pode-se imaginar, tal resultado consiste numa condição suficiente para que um operador seja hipercíclico. É natural, então, perguntar: seria também esta condição necessária? C.J. Read e Manuel de la Rosa mostraram que não, construindo um espaço de Banach conveniente que possui um operador hipercíclico que não satisfaz o Critério. Pouco tempo depois, Frédéric Bayart e Étienne Matheron apresentaram um contraexemplo nos espaços $c_{0}$ e $\ell_{p}$, $1 \leq p<\infty$.

Apesar destes e de outros resultados, o conceito de sub-hiperciclicidade surgiu apenas em 2011, sendo definido por Madore e Martínez-Avendaño em [18]. Antes disso, subespaços apareciam apenas no estudo do conjunto de vetores hipercíclicos, de maneira similar ao já citado Teorema de HerreroBourdon.

À primeira vista, o estudo de operadores sub-hipercíclicos pode parecer trivial: é natural assumir que eles devem se comportar, em grande parte, da mesma maneira que operadores hipercíclicos. Como veremos ao longo desta tese, na maioria das vezes isto é verdade - e talvez isso responda a pergunta do porquê o conceito de sub-hiperciclicidade ter sido definido apenas recentemente. No entanto, generalizar resultados de operadores hipercíclicos para sub-hipercíclicos nem sempre é trivial: vale ressaltar na definição de sub-hiperciclicidade que orb $(x, T)$ precisa ser denso, mas não necessariamente contido, em $M$. Este detalhe faz com que os resultados e as demonstrações em hiperciclicidade não se traduzam "automaticamente" para operadores sub-hipercíclicos - e a

\footnotetext{
${ }^{1}$ Isto é, $M \neq X$ e $M \neq\{0\}$.
} 
partir daí algumas adaptações são necessárias. Para exemplificar a dificuldade de se trabalhar com operadores sub-hipercíclicos, a pergunta mais imediata possível - será que todo operador hipercíclico é sub-hipercíclico? - foi respondida apenas em 2016, cinco anos após a introdução do conceito de sub-hiperciclicidade.

Um outro pequeno detalhe que torna a generalização de resultados um tanto quanto difícil é a separabilidade: se um espaço de Banach admite um operador hipercíclico, não é difícil observar que este espaço precisa ser separável. Porém, este não necessariamente é o caso com operadores subhipercíclicos já que, como é de se imaginar, apenas o subespaço em questão precisa ser separável ${ }^{2}$ Com a ausência da separabilidade no espaço inteiro, algumas das técnicas utilizadas para demonstrar resultados importantes para operadores hipercíclicos não se traduzem para o contexto de operadores sub-hipercíclicos.

Levando em conta tudo que foi dito, ainda há muitos problemas em aberto para operadores sub-hipercíclicos. Nesta tese nós apresentamos alguns novos resultados para operadores subhipercíclicos, muitos dos quais são inspirados ou adaptados (levando em conta as restrições impostas, como dito nos parágrafos anteriores) de resultados conhecidos em hiperciclicidade. No primeiro capítulo apresentaremos alguns resultados básicos em espaços de Banach, necessários para o completo entendimento deste trabalho, assim como alguns resultados clássicos em hiperciclicidade (como aqueles citados anteriormente). No segundo capítulo faremos uma introdução mais detalhada ao conceito de sub-hiperciclicidade, apresentaremos alguns exemplos e mostraremos alguns novos resultados, entre os quais destacamos o Teorema 2.17 (em um espaço de Banach separável, dado um subespaço fechado e de dimensão infinita $M$, existe um operador que satisfaz o Critério e é sub-hipercíclico para $M$ ) e o Teorema 2.31 (todo espaço de Banach admite um operador subhipercíclico).

No terceiro capítulo apresentaremos um Critério de Sub-Hiperciclicidade. Este resultado, claramente inspirado no resultado análogo em hiperciclicidade, foi mostrado pela primeira vez em [18] e fornece um critério para que um operador seja sub-hipercíclico. Assim como em hiperciclicidade, surge a pergunta natural: todo operador sub-hipercíclico satisfaz o Critério de Sub-Hiperciclicidade? Apresentamos uma resposta a esta pergunta nesse capítulo. Além disso, demonstramos no Teorema 3.14 um outro critério para sub-hiperciclicidade.

No Capítulo 4, tornamos nossa atenção para um outro problema. Dissemos que nem todo operador hipercíclico satisfaz o Critério de Hiperciclicidade. Cabe, então, a seguinte pergunta: existe um espaço de Banach onde todo operador hipercíclico satisfaz o Critério? O Teorema de Ansari-Bernal nos mostra que todo espaço de Banach separável contém um operador hipercíclico. Além disso, apesar de não termos dito anteriormente, o teorema também garante que tal operador, que é da forma $I+K$ com $K$ compacto, satisfaz o Critério. Sabendo que existem operadores hipercíclicos da forma $I+K$ que satisfazem o Critério (e também sabendo que os contraexemplos apresentados por Read/de la Rosa e Bayart/Matheron não contém operadores desta classe), somos levados a nos perguntar: será que todo operador hipercíclico da forma $I+K$ satisfaz o Critério?

Ao tentarmos responder tais perguntas, somos levados a estudar os chamados espaços com pouquíssimos operadores. Estes espaços são conhecidos por terem a propriedade de que todo opera-

\footnotetext{
${ }^{2}$ Apenas para complementar e exemplificar, veremos no simples Exemplo 2.3 que existe um operador $T \in \mathcal{B}\left(\ell_{\infty}\right)$ que é $c_{0}$-hipercíclico.
} 
dor limitado pode ser escrito na forma $\lambda I+K$, onde $K$ é um compacto. Como não é difícil perceber, temos que se $\lambda I+K$ é hipercíclico, então o operador $K$ é cíclico. Logo, estudar a hiperciclicidade de $\lambda I+K$ está diretamente relacionada com estudar a ciclicidade de $K$. Dito isso, estudaremos as relações entre ciclicidade e hiperciclicidade, motivados por tal classe de operadores. Apresentamos também um Critério de Ciclicidade que, mais uma vez, é motivado pelo resultado equivalente para operadores hipercíclicos. Veremos também que a construção de Bayart e Matheron não pode ser replicada nos tais espaços com pouquíssimos operadores. Para encerrar tal capítulo, vamos definir a noção de sub-ciclicidade - claramente motivados pelo conceito de sub-hiperciclicidade - e apresentar alguns resultados, entre eles o Critério de Sub-Ciclicidade

Por fim, encerramos a tese com um capítulo debatendo os problemas aqui discutidos e levantando outras questões a serem estudadas em futuras pesquisas.

\section{Sobre os resultados nesta tese}

De modo a ser transparente, com exceção do primeiro capítulo, todos os resultados não-originais (até as definições) estarão acompanhados de uma referência em seu cabeçalho (que não necessariamente será o artigo original, de maneira a não estender demasiadamente a bibliografia). 


\section{Capítulo 1}

\section{Preliminares}

Como estamos interessados em estudar hiperciclicidade (e conceitos derivados) em espaços de Banach, vamos apresentar neste capítulo alguns resultados relativos a estes espaços que serão utilizados e citados ao longo do texto. Além disso, para aqueles não familiares com o assunto, vamos também apresentar as noções básicas de hiperciclicidade e alguns resultados interessantes dentro do tema e que serão explorados em outros capítulos. Todos os resultados aqui apresentados (tanto de espaços de Banach como de hiperciclicidade) são conhecidos dentro da literatura.

Vale observar que as demonstrações sugeridas nos resultados deste capítulo nem sempre são dos autores originais. Optamos por concentrar as referências dos resultados envolvendo espaços de Banach nos livros [2] e [22], enquanto as referências dos resultados envolvendo hiperciclicidade estão, em sua maioria, concentradas no livro [14].

Em alguns dos resultados, optamos por exibir uma demonstração ao invés de colocar uma referência. Isto foi feito por um dos dois motivos: tal demonstração será um ponto de discussão ao longo do texto ou por não termos encontrado uma referência em que o resultado em questão tivesse uma demonstração clara ou acessível.

Por fim, ao dizermos durante o texto que um operador linear contínuo $T$ é invertível, estamos considerando que $T$ é uma bijeção com sua inversa $T^{-1}$ contínua.

\subsection{Espaços de Banach}

Começamos esta seção com alguns resultados gerais em espaços de Banach:

Definição 1.1. Sejam $X$ um espaço de Banach e $T \in \mathcal{B}(X)$. $O$ espectro pontual de $T$ é o conjunto

$$
\sigma_{p}(T):=\{\alpha \in \mathbb{K}: \operatorname{ker}(T-\alpha I) \neq\{0\}\}
$$

Antes do próximo lema, vale lembrar que se $T \in \mathcal{B}(X)$, então o adjunto de $T$ é o operador linear contínuo $T^{*}: X^{*} \rightarrow X^{*}$ definido por $T^{*}\left(x^{*}\right):=x^{*} \circ T$. Além disso, o complemento ortogonal do espaço $T(X)$ é o subespaço de $X^{*}$ definido por $T(X)^{\perp}:=\left\{x^{*} \in X^{*}: x^{*}(y)=0\right.$, para todo $y \in$ $T(X)\}$

Lema 1.2. Sejam $X$ um espaço de Banach e $T \in \mathcal{B}(X)$. O operador $T^{*}$ é injetor se, e somente se, a imagem de $T$ é densa em $X$. 
DemonstraçÃo: Note que

$$
\begin{aligned}
\operatorname{ker}\left(T^{*}\right) & =\left\{x^{*}: T^{*}\left(x^{*}\right)=0\right\} \\
& =\left\{x^{*} \in X^{*}: T^{*}\left(x^{*}\right)(y)=0, \text { para todo } y \in X\right\} \\
& =\left\{x^{*} \in X^{*}: x^{*}(T(y))=0, \text { para todo } y \in X\right\} \\
& =T(X)^{\perp}
\end{aligned}
$$

De posse da igualdade mostrada acima, sabendo que o $T^{*}$ é injetivo se, e somente se, $\operatorname{ker}\left(T^{*}\right)=$ $\{0\}$ e que $T(X)^{\perp}=\{0\}$ se, e somente se, $T(X)$ é denso em $X$, obtemos o desejado.

O próximo lema garante que qualquer par de sequências densas e linearmente independentes são isomorficamente equivalentes.

Lema 1.3 (Lema 8.22 de [14]). Sejam $X$ um espaço de Banach, $X_{0}$ e $Y_{0}$ conjuntos enumeráveis, densos e linearmente independentes em $X$. Tome $\varepsilon>0$. Então existe um operador linear invertivel $A$ em $X$ tal que $A\left(X_{0}\right)=Y_{0}$ e $\|I-A\|<\varepsilon$.

Lembremos agora que se $V$ é um espaço vetorial e $W$ um subespaço vetorial de $V$, então a codimensão de $W$ em $V$ (denotada por codim $W$ ) é a dimensão do espaço quociente $V / W$.

Lema 1.4 (Lema 3.4.20 de [22]). Seja $X$ um espaço de Banach e tome $T \in \mathcal{B}(X)$. Se $T=$ $\lambda I+K$, onde $\lambda \neq 0$ e $K$ é compacto, então $\operatorname{dim} \operatorname{ker} T<\infty$ e $T$ tem imagem fechada. Além disso, $\operatorname{dim} \operatorname{ker} T=\operatorname{codim} \operatorname{Im} T$.

O próximo resultado é um famoso teorema cuja autoria é, em geral, atribuída à Mazur:

Teorema 1.5 (Teorema 4.1.30 de [22]). Seja, X um espaço de Banach de dimensão infinita e $M>1$. Então existe uma sequência básica normalizada em $X$ com constante básica menor ou igual $\grave{a} M$.

A seguir, temos dois resultados envolvendo o conhecido espaço $\ell_{\infty}$. Para o primeiro, note que um operador linear contínuo $\phi: X \rightarrow Y$ é uma imersão isométrica se $\|\phi(x)\|_{Y}=\|x\|_{X}$, para todo $x \in X$. Neste caso, $\phi$ não é necessariamente sobrejetor.

Teorema 1.6 (Teorema 2.5.7 de [2]). Seja X um espaço de Banach separável. Então existe uma imersão isométrica de $X$ em $\ell_{\infty}$.

Proposição 1.7. $\left(\ell_{\infty}\right)^{*}=\ell_{1} \oplus c_{0}^{\perp}$.

DemonstraÇão: Sejam $f \in\left(\ell_{\infty}\right)^{*}$ e $a_{i}:=f\left(e_{i}\right)$, para todo $i \in \mathbb{N}$, onde $e_{i}$ é o vetor canônico em $\ell_{\infty}$. Tomando $\varepsilon_{i}= \pm 1$ de acordo com o sinal de $f\left(e_{i}\right)$ (isto é, $\left.\left|f\left(e_{i}\right)\right|=\varepsilon_{i} f\left(e_{i}\right)\right)$ 1, vamos mostrar agora que a sequência $a:=\left(a_{i}\right)_{i \in \mathbb{N}} \in \ell_{1}$ :

$$
\sum_{i \in \mathbb{N}}\left|a_{i}\right|=\lim _{n \rightarrow \infty} \sum_{i=1}^{n}\left|a_{i}\right|=\lim _{n \rightarrow \infty} \sum_{i=1}^{n}\left|f\left(e_{i}\right)\right|=\lim _{n \rightarrow \infty} f\left(\sum_{i=1}^{n} \varepsilon_{i} e_{i}\right) \leq \lim _{n \rightarrow \infty}\|f\|=\|f\|
$$

\footnotetext{
${ }^{1}$ Estamos aqui considerando, implicitamente, que estamos trabalhando com espaços de Banach reais. No entanto, não é difícil usar o mesmo argumento no caso de espaços de Banach complexos: se escrevermos $a_{j}=r_{j} \mathrm{e}^{i \theta_{j}}$, então $\frac{1}{\mathrm{e}^{i \theta_{j}}} \cdot f\left(e_{j}\right)=r_{j}$. Com isso, basta reescrever a Equação 1.1 usando $\left|a_{j}\right|=r_{j}=\frac{1}{\mathrm{e}^{i \theta_{j}}} \cdot f\left(e_{j}\right)$, para todo $j \in \mathbb{N}$.
} 
uma vez que $\left\|\sum_{i=1}^{n} \varepsilon_{i} e_{i}\right\|_{\infty}=1$.

Seja agora $x=\left(x_{n}\right)_{n \in \mathbb{N}} \in c_{0} \subseteq \ell_{\infty}$. Logo, podemos escrever $x=\sum_{i \in \mathbb{N}} x_{i} e_{i}$, onde $e_{i}$ é o vetor canônico de $c_{0}$. Como $c_{0}^{*} \equiv \ell_{1}$, então $a \in c_{0}^{*}$. Temos:

$$
\begin{aligned}
f(x)-a(x) & =f\left(\sum_{i \in \mathbb{N}} x_{i} e_{i}\right)-a\left(\sum_{i \in \mathbb{N}} x_{i} e_{i}\right)=\lim _{n \rightarrow \infty}\left[f\left(\sum_{i=1}^{n} x_{i} e_{i}\right)-a\left(\sum_{i=1}^{n} x_{i} e_{i}\right)\right] \\
& =\lim _{n \rightarrow \infty}\left(\sum_{i=1}^{n} x_{i} f\left(e_{i}\right)-\sum_{i=1}^{n} x_{i} a_{i}\right)=\lim _{n \rightarrow \infty} \sum_{i=1}^{n}\left(x_{i} a_{i}-x_{i} a_{i}\right) \\
& =0
\end{aligned}
$$

uma vez que $a_{i}=f\left(e_{i}\right)$. Logo, $f-a \in c_{0}^{\perp}$ e como $f=a+(f-a)$, isto que mostra que $\left(\ell_{\infty}\right)^{*}=\ell_{1} \oplus c_{0}^{\perp}$.

O próximo teorema é um resultado clássico de Ovsepian e Pełczyński e que será de fundamental importância na demonstração da existência de operadores hipercíclicos:

Teorema 1.8 (Ovsepian e Pełczyński, [23]). Seja X um espaço de Banach separável de dimensão infinita. Então existe $\left\{x_{n}\right\}_{n=1}^{\infty} \subseteq X$ e $\left\{x_{n}^{*}\right\}_{n=1}^{\infty} \subseteq X^{*}$ tal que

(a) $x_{i}^{*}\left(x_{j}\right)=\delta_{i j}$, onde $\delta_{i j}$ é o delta de Kronecker.

(b) $\operatorname{span}\left\{x_{n}: n \in \mathbb{N}\right\}$ é denso em $X$.

(c) se $x_{n}^{*}(x)=0$ para todo $n \in \mathbb{N}$, então $x=0$.

(d) $\left\|x_{n}\right\|=1$, para todo $n \in \mathbb{N} e \sup _{n \in \mathbb{N}}\left\|x_{n}^{*}\right\|=C<\infty$.

Vamos agora lembrar rapidamente o conceito de soma direta externa:

Definição 1.9. Sejam $X_{1}, \ldots, X_{n}$ espaços normados com respectivas normas $\|\cdot\|_{X_{1}}, \ldots,\|\cdot\|_{X_{n}} \cdot A$ soma direta externa (ou produto direto) de $X_{1}, \ldots, X_{n}$ é o espaço vetorial $X_{1} \times \ldots \times X_{n}$ (com as operações usuais) munido da norma

$$
\left\|\left(x_{1}, \ldots, x_{n}\right)\right\|=\left(\sum_{j=1}^{n}\left\|x_{j}\right\|_{X_{j}}^{2}\right)^{1 / 2}
$$

Tal espaço normado é denotado por $X_{1} \oplus \ldots \oplus X_{n}$.

Embora utilizem a mesma notação, vale ressaltar que a soma direta externa é, em teoria, diferente daquela utilizada na Proposição 1.7 (a soma direta interna). No entanto, como pode-se ver na Proposição 1.8.10 de [22], ambas são equivalentes - e por isso não há um conflito de notações.

Se $T \in \mathcal{B}(X)$ e $S \in \mathcal{B}(Y)$, definimos naturalmente o operador linear contínuo $T \oplus S: X \oplus Y \rightarrow$ $X \oplus Y$ por $(T \oplus S)(x, y):=(T x, S y)$. Ainda, se $X_{1}, \ldots X_{n}$ forem espaços de Banach, então $X_{1} \oplus \ldots \oplus X_{n}$ é um espaço de Banach (vide o Teorema 1.8.6 de [22]).

Por fim, apresentaremos agora a definição de espaços de Banach injetivos e apresentamos uma proposição que mostra que $\ell_{\infty}$ tem essa propriedade: 
Definição 1.10. Um espaço de Banach $Y$ é dito injetivo se, para todo espaço de Banach $X$, subespaço fechado $W \subseteq X$ e $T: W \rightarrow Y$ operador linear limitado, então existe um operador linear limitado $\tilde{T}: X \rightarrow Y$ que estende $T$. Dizemos ainda que $Y$ é isometricamente injetivo se podemos tomar $\tilde{T}$ tal que $\|\tilde{T}\|=\|T\|$.

Proposição 1.11 (Proposição 2.5.2 de [2]). O espaço $\ell_{\infty}$ é isometricamente injetivo.

\subsection{Hiperciclicidade}

Seja $E$ um espaço vetorial topológico qualquer. É bem conhecido que um operador linear contínuo $T: E \rightarrow E$ é dito cíclico se existir um vetor $y \in E$ tal que span $\left\{y, T y, T^{2} y, \ldots T^{n} y \ldots\right\}$ é denso em $E$. Tal vetor $y$ é dito vetor cíclico para $T$. Tendo ciclicidade em mente, podemos ir além e exigir apenas que o conjunto $\left\{y, T y, T^{2} y, \ldots T^{n} y \ldots\right\}$ fosse denso em $E$. Isto nos remete ao conceito de hiperciclicidade. Apesar de já termos colocado a definição na introdução, vamos colocá-la de novo a seguir, de maneira a deixarmos o texto completo.

Definição 1.12. Seja $X$ um espaço de Banach. Um operador linear limitado $T: X \rightarrow X$ é dito hipercíclico se existir um vetor $x \in X$ tal que

$$
\operatorname{orb}(x, T):=\left\{x, T(x), T^{2}(x), T^{3}(x), \ldots T^{n}(x) \ldots\right\}
$$

é denso em $X$. O conjunto orb $(x, T)$ é a órbita de $x$ (por $T$ ) enquanto o vetor $x$ é dito vetor hipercíclico para $T$. Denotaremos por $H(T)$ o conjunto dos vetores hipercíclicos de $T$.

Com a definição acima em mente e utilizando o Lema 1.4, temos o seguinte corolário imediato:

Corolário 1.13. Se $T=I+K$ é hipercíclico, onde $K$ é compacto, então $T$ é invertível.

É imediato ver que todo operador hipercíclico também é cíclico. Pela similaridade das definições, temos que ambos os conceitos não só compartilham propriedades mas como se relacionam de diversas maneiras. Veremos tais relações ao longo do texto

Note que hiperciclicidade é fundamentalmente um conceito topológico, isto é, depende apenas da topologia do espaço. Por isso, tal definição ainda é válida se trocarmos a hipótese de $X$ ser Banach por $X$ ser um espaço de Fréchet ou até mesmo um espaço vetorial topológico, por exemplo. Vale frisar que, apesar de nosso foco neste trabalho é estudar hiperciclicidade (e conceitos derivados) em espaços de Banach, a pesquisa de hiperciclicidade em espaços de Fréchet ou EVT's também é frutífera. Por exemplo, maioria dos resultados citados nesta seção também vale para espaços de Fréchet. Já no caso de hiperciclicidade em espaços vetoriais topológicos, indicamos como referência o Capítulo 12 de [14] para os interessados no estudo de resultados em tais espaços.

É imediato ver que um operador hipercíclico tem imagem densa. Além disso, se $x$ é um vetor hipercíclico para $T$, então $T^{n} x$ também o é, para todo $n \in \mathbb{N}$. De fato, se $X$ é um espaço de Banach, então $X$ não possui pontos isolados. Portanto, podemos remover uma quantidade finita de pontos de um conjunto denso de maneira a obtermos um conjunto que continue sendo denso. Ou seja, se orb $(x, T)=\left\{x, T(x), T^{2}(x), \ldots, T^{n}(x), T^{n+1}(x), \ldots\right\}$ é denso em $X$, então temos que 
$\left\{T^{n}(x), T^{n+1}(x), T^{n+2}(x), \ldots\right\}=\operatorname{orb}\left(T^{n} x, T\right)$ também é denso em $X$, o que mostra que $T^{n} x$ é um vetor hipercíclico para $T{ }^{2}$

Como orb $(x, T)$ é denso em $X$ e todo $T^{n} x$ é um vetor hipercíclico para $T$, imediatamente chegamos a conclusão de que $H(T)$ é um conjunto denso em $X$. Como veremos no Teorema da Transitividade de Birkhoff a seguir, tal conjunto é um $G_{\delta}$ denso em $X$. Não só isso, mas $H(T) \cup\{0\}$ contém um subespaço de dimensão infinita. Para mais detalhes, consultar a Seção 2.6 de [14].

Os primeiros exemplos de operadores hipercíclicos foram dados por G. D. Birkhoff (1929), G.R. MacLane (1952) e S. Rolewicz (1969). Considerando $\mathcal{H}(\mathbb{C})$ como sendo o espaço de todas as funções complexas holomorfas, Birkhoff mostrou que o operador $T_{a}: \mathcal{H}(\mathbb{C}) \rightarrow \mathcal{H}(\mathbb{C})$ definido por $T_{a}(f)(z)=f(z+a)$ é hipercíclico. Já MacLane mostrou que o operador derivação definido em $\mathcal{H}(\mathbb{C})$ também é hipercíclico. O exemplo de Rolewicz será discutido mais tarde.

Em todos estes exemplos os autores construíram os vetores hipercíclicos explicitamente e mostraram que sua órbita era densa, usando propriedades dos espaços e dos operadores em questão. No entanto, isso nem sempre é uma tarefa fácil: dado um operador $T$, seria interessante se existisse um critério que dependesse apenas do operador em si para verificar se $T$ é hipercíclico. Com isso, não seria necessária a construção explícita de um vetor hipercíclico $x$ para $T$ - nem demonstrar que tal vetor tem órbita densa. Como é comum em outras áreas da análise, estamos preocupados com a existência (de vetores hipercíclicos), e não com a sua "identidade".

Nesse sentido, em 1982 Carol Kitai apresentou, em sua tese de doutorado, uma condição suficiente para hiperciclicidade. Esse resultado é hoje conhecido como Critério de Kitai. Embora na demonstração de tal critério Kitai tenha construído explicitamente um vetor para mostrar que o operador em questão é hipercíclico (ao contrário do que desejamos, como dito no parágrafo anterior), o critério em si não exige essa construção. Apesar deste avanço importante, Kitai nunca publicou seu resultado.

Com o passar dos anos, esse critério foi sendo refinado e aprimorado até chegar à forma que apresentaremos: o conhecido Critério de Hiperciclicidade. Este enunciado é devido à Bés e Peris, vide [8].

\subsubsection{O Critério de Hiperciclicidade}

Teorema 1.14 (Critério de Hiperciclicidade). Sejam X um espaço de Banach separável de dimensão infinita e $T: X \rightarrow X$ um operador linear limitado. Suponha que existem subconjuntos $X_{0}, Y_{0}$ densos em $X$, uma sequência crescente de inteiros positivos $\left(n_{k}\right)_{k \in \mathbb{N}}$ e funções $S_{n_{k}}: Y_{0} \rightarrow X$ tais que:

(i) $T^{n_{k}}(x) \rightarrow 0$, para todo $x \in X_{0}$.

(ii) $S_{n_{k}}(y) \rightarrow 0$, para todo $y \in Y_{0}$.

(iii) $T^{n_{k}} \circ S_{n_{k}}(y) \rightarrow y$, para todo $y \in Y_{0}$.

Então T é hipercíclico.

\footnotetext{
${ }^{2}$ Como espaços de Fréchet não possuem pontos isolados, então o que acabamos de concluir também é válido em tais espaços, indo de acordo com o que foi dito no parágrafo anterior.
} 
Observação 1.15. Podemos reescrever as condições (ii) e (iii) da seguinte maneira: "dado $y \in Y_{0}$, existe uma sequência $\left(x_{k}\right)_{k \in \mathbb{N}} \subseteq X$ tal que $x_{k} \rightarrow 0$ e $T^{n_{k}} x_{k} \rightarrow y$ ". Para mostrar a equivalência entre esta condição e as condições (ii) e (iii) do Critério de Hiperciclicidade, basta definir $x_{k}:=S_{n_{k}} y$ (e vice-versa).

Durante o restante do texto, tomaremos a liberdade de fazer referência ao Critério de Hiperciclicidade apenas por Critério ou pela sigla $\mathbf{H C}]^{3}$

A demonstração do Critério de Hiperciclicidade será dada mais adiante uma vez que faremos uso de outros conceitos (que veremos a seguir) em sua demonstração. Vale destacar que, na demonstração original dada por Kitai, ela utilizou as condições (i), (ii) e (iii) para construir um vetor hipercíclico para T. Para uma demonstração do Critério baseada na demonstração original de Kitai, veja [14, pp.75-76].

Curiosamente, o resultado hoje conhecido como Critério de Kitai nada mais é do que o Critério de Hiperciclicidade com duas alterações: ao invés de exigir uma sequência crescente $\left(n_{k}\right)_{k \in \mathbb{N}}$, o Critério de Kitai exige que as condições (i) e (ii) do HC sejam satisfeitas para a sequência $(n)_{n \in \mathbb{N}}$ dos números naturais. Já a condição (iii) do HC é substítuida no Critério de Kitai pela condição " $T \circ S(y)=y$, para todo $y \in Y_{0}$ ".

Por fim, observe que no Critério de Hiperciclicidade não há necessidade das funções $S_{n_{k}}$ serem lineares nem contínuas. Isto faz com que, desde que as funções $S_{n_{k}}$ satisfaçam (ii) e (iii), não precisemos nos preocupar com o modo que construímos tais funções.

Vejamos agora outro conceito que, como mostra o teorema após a definição, tem tudo a ver com hiperciclicidade:

Definição 1.16. Sejam $X$ um espaço de Banach separável de dimensão infinita e $T \in \mathcal{B}(X)$. Dizemos que $T$ é topologicamente transitivo se dados $U, V$ abertos não-vazios de $X$ existe $n \in \mathbb{N}$ tal que $T^{n}(U) \cap V \neq \emptyset$.

Teorema 1.17 (Teorema da Transitividade de Birkhoff). Sejam X um espaço de Banach separável de dimensão infinita e $T \in \mathcal{B}(X)$. Então, são equivalentes:

(i) T é topologicamente transitivo.

(ii) Té um operador hipercíclico.

Neste caso, o conjunto dos vetores hipercíclicos de $T$ é um conjunto $G_{\delta}$ denso em $X$.

DemonstraÇÃo: Ver [14, Teorema 2.19, p.39].

Como veremos nos próximos capítulos, a equivalência entre transitividade e hiperciclicidade não continua válida quando pensamos em sub-hiperciclicidade e sub-transitividade - e a ausência de tal equivalência tem consequências quando tentamos formular um Critério de Sub-Hiperciclicidade.

${ }^{3}$ Do inglês, Hypercyclicity Criterion. 
De maneira a elucidar melhor tais consequências - e cumprindo a promessa da demonstração feita anteriormente - utilizaremos agora o Teorema da Transitividade de Birkhoff para apresentar uma demonstração do Critério de Hiperciclicidade:

Demonstração: Sejam $U, V$ dois abertos não-vazios de $X$. Pela densidade de $X_{0}$ e $Y_{0}$, existem $x, y$ tais que $x \in X_{0} \cap U$ e $y \in Y_{0} \cap V$. Como $x \in X_{0}$, então $T^{n_{k}}(x) \rightarrow 0$, pela condição (i) da hipótese. Da mesma maneira, da condição (ii) temos que $S_{n_{k}}(y) \rightarrow 0$. Logo, definindo $x_{k}:=x+S_{n_{k}}(y)$, temos que $x_{k} \rightarrow x$. Portanto, como $x \in U$, existe um $k_{1} \in \mathbb{N}$ tal que, para todo $k>k_{1}, x_{k} \in U$. Sendo $T$ um operador linear, temos que

$$
T^{n_{k}}\left(x_{k}\right)=T^{n_{k}}\left(x+S_{n_{k}}(y)\right)=T^{n_{k}}(x)+T^{n_{k}} \circ S_{n_{k}}(y) \rightarrow y
$$

usando as condições (i) e (iii) da hipótese. Logo, como $y \in V$, existe um $k_{2} \in \mathbb{N}$ tal que, para todo $k>k_{2}$, devemos ter $T^{n_{k}}\left(x_{k}\right) \in V$.

Dessa forma, tomando um $k_{0} \geq \max \left\{k_{1}, k_{2}\right\}$, temos que, para todo $k>k_{0}$, ambos $x_{k} \in U$ e $T^{n_{k}}\left(x_{k}\right) \in V$ são satisfeitos. Portanto, segue que $T^{n_{k}}(U) \cap V \neq \emptyset$, para todo $k>k_{0}$, o que prova que $T$ é topologicamente transitivo. Logo, pelo Teorema da Transitividade de Birkhoff, $T$ é hipercíclico, como desejado.

O exemplo a seguir foi o primeiro exemplo conhecido de um operador hipercíclico nos espaços de Banach clássicos, a saber, $c_{0}$ e $\ell_{p}, 1 \leq p<\infty$. Ele foi dado por S. Rolewicz em 1969. No entanto, ao contrário do que mostraremos no exemplo, Rolewicz não usou o Critério de Hiperciclicidade (que, como já mencioamos, apareceu em sua primeira versão apenas nos anos 80 com o Critério de Kitai) para mostrar que o operador em questão era hipercíclico: ele construiu um vetor $x_{0}$ e mostrou, usando apenas a definição de densidade, que a órbita de $x_{0}$ era densa. A demonstração feita por Rolewicz pode ser encontrada em [25, Teorema 1, pp.17-19].

Exemplo 1.18. Sejam $X=c_{0}$ ou $\ell_{p}$, com $1 \leq p \leq \infty$, e $w \in \mathbb{K}$ tal que $|w|>1$. O backward shift

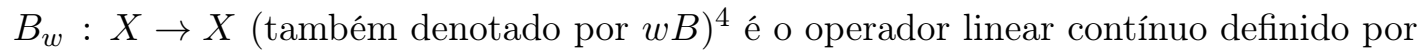

$$
B_{w}\left(x_{1}, x_{2}, x_{3}, \ldots\right)=w \cdot\left(x_{2}, x_{3}, x_{4}, \ldots\right)
$$

Vamos usar o Critério de Hiperciclicidade para provar que $B_{w}$ é hipercíclico em $c_{0}$ e $\ell_{p}$, para $1 \leq p<\infty]^{5}$ No que segue, tomaremos $X=c_{0}$ ou $\ell_{p}, 1 \leq p<\infty$; a demonstração para ambos os casos é igual.

Sejam $X_{0}$ e $Y_{0}$ o espaço das sequências em $X$ com um número finito de coordenadas não-nulas (isto é, $X_{0}=Y_{0}=c_{00}$ ). É claro que $X_{0}$ e $Y_{0}$ são densos em $X$. Defina o operador $S_{w^{-1}}: Y_{0} \rightarrow Y_{0}$ dado por $S_{w^{-1}}\left(x_{1}, x_{2}, x_{3}, \ldots\right)=w^{-1} \cdot\left(0, x_{1}, x_{2}, \ldots\right)$. Tomaremos $\left(n_{k}\right)_{k \in \mathbb{N}}=(n)_{n \in \mathbb{N}}$ como sendo a sequência exigida pelo Critério. Verificaremos agora que (i), (ii) e (iii) do Critério são satisfeitos.

\footnotetext{
${ }^{4}$ Por vezes usaremos a notação $w B$ ao invés de $B_{w}$, especialmente quando o forward shift $F$ também estiver no contexto (pois, dessa maneira, é muito mais fácil de visualizar que $(w B) \circ\left(w^{-1} F\right)=I$ ).

${ }^{5}$ Note que, apesar de termos definido $B_{w}$ em $\ell_{\infty}$, não é possível mostrar que $B_{w}$ é hipercíclico em tal espaço, uma vez que $\ell_{\infty}$ não é separável - uma condição necessária para a existência de operadores hipercíclicos, como discutiremos adiante.
} 
Pela definição de $B_{w}$ e de $X_{0}$ é claro que (i) é satisfeito. Como $B_{w} \circ S_{w^{-1}}=I d$, então é óbvio que (iii) também é satisfeito. Resta então provarmos (ii). Se $x=\left(x_{1}, x_{2}, x_{3}, \ldots\right) \in Y_{0}$, note que

$$
\left\|S_{w^{-1}}^{n}\left(x_{1}, x_{2}, \ldots\right)\right\|=\frac{1}{|w|^{n}} \| \underbrace{(0,0, \ldots, 0}_{n \text { coordenadas }}, x_{1}, x_{2}, \ldots)\left\|=\frac{1}{|w|^{n}}\right\| x \| \rightarrow 0
$$

quando $n \rightarrow \infty$, visto que $x$ está fixo e $|w|>1$. Logo, $S_{w^{-1}}$ satisfaz a segunda condição, o que mostra que $B_{w}$ é hipercíclico.

Em [14, Exemplo 3.7, p.72] é verificado, utilizando o Critério de Kitai, que o operador derivação em $\mathcal{H}(\mathbb{C})$ é hipercíclico; como dito anteriormente, tal exemplo foi obtido sem a ajuda do Critério de Kitai (ou do Critério de Hiperciclicidade) por MacLane em 1952. Outros exemplos podem ser vistos no Capítulo 4 de [14].

Como dissemos na introdução, o Critério de Hiperciclicidade consiste numa condição suficiente para que um operador $T$ seja hipercíclico. Quase que imediatamente surge a pergunta: será esta condição também necessária? Isto é, será que todo operador hipercíclico satisfaz o Critério? Tal problema foi, por muitos anos, o principal problema em aberto na teoria dos operadores hipercíclicos.

Em 2006, finalmente C. J. Read e Manuel de la Rosa resolveram esse problema. Em [10], eles construíram uma norma apropriada em um espaço de Banach onde é possível encontrar um operador hipercíclico $T$ que não satisfaz o Critério de Hiperciclicidade. Baseada na construção de De La Rosa e Read, Frédéric Bayart e Étienne Matheron construíram um contraexemplo nos espaços de Banach clássicos, a saber, $c_{0}$ e $\left.\ell_{p}, 1 \leq p<\infty\right]^{6}$ Para facilitar futuras referências, colocamos o resultado de Bayart e Matheron na forma de teorema:

Teorema 1.19 (Bayart e Matheron, [7]). Existem operadores hipercíclicos em $c_{0}$ e $\ell_{p}, 1 \leq p<\infty$ que não satisfazem o Critério de Hiperciclicidade.

Bayart e Matheron (assim como De La Rosa e Read) se utilizaram de uma equivalência para mostrar que um operador hipercíclico não satisfaz o Critério, uma vez que mostrar diretamente que um operador hipercíclico não satisfaz o Critério é uma tarefa bastante complicada. Tal equivalência é, de novo, devida à Bès e Peris:

Teorema 1.20 (Bès e Peris, [8]). Seja T um operador hipercíclico em um espaço de Banach. São equivalentes:

(i) T satisfaz o Critério de Hiperciclicidade.

(ii) $T \oplus T$ é hipercíclico.

(iii) $T \oplus T$ é cíclico.

DemonstraçÃo: Este teorema é uma junção dos resultados demonstrados em [8, Teorema 2.5, p.98] e [13, Proposição 4.1, p. 159].

\footnotetext{
${ }^{6}$ Apesar de o artigo de Bayart e Matheron ser anterior, um preprint com a construção de De La Rosa e Read já estava em circulação, como Bayart e Matheron dizem (e referenciam) em seu próprio artigo.
} 
Uma vez respondida essa pergunta, vamos agora propor outra: em quais espaços existem operadores hipercíclicos? Usando a definição de hiperciclicidade, não é difícil observar que o espaço em questão precisa ser separável. Além disso, ao contrário do que acontece com ciclicidade, hiperciclicidade é um fenômeno exclusivo de dimensão infinita.7] Tal resultado pode ser visto em [14, Teorema 2.58, p.55].

Temos, então, que ter dimensão infinita e separabilidade são condições necessárias para que um espaço tenha um operador hipercíclico. Seriam elas suficientes? Ou será que existem outras condições? Como veremos na seção a seguir, as duas condições citadas se mostram suficientes para garantir a existência de operadores hipercíclicos em espaços de Banach.

\subsection{Existência de Operadores Hipercíclicos}

Antes de apresentarmos o teorema que trata da existência de operadores hipercíclicos, vejamos alguns conceitos auxiliares que serão úteis ao longo do texto:

Definição 1.21. Seja $X$ um espaço de Banach. Um operador linear limitado $T: X \rightarrow X$ é dito mixing se, para qualquer par $U, V$ de abertos não-vazios de $X$ existe $k \in \mathbb{N}$ tal que $T^{n}(U) \cap V \neq \emptyset$, para todo $n \geq k$.

Vejamos um exemplo que será importante mais adiante:

Exemplo 1.22. Sejam $X=c_{0}$ ou $\ell_{p}$, para $1 \leq p<\infty$ e $\left(w_{n}\right)_{n \in \mathbb{N}} \subseteq \mathbb{K}$ uma sequência tal que $\sup \left|w_{n}\right|<\infty$. Então o operador $I+B_{w_{n}}$, onde $I$ é a identidade em $X$ e $B_{w_{n}} \in \mathcal{B}(X)$ é definido por $B_{w_{n}}\left(x_{1}, x_{2}, \ldots\right)=\left(w_{2} x_{2}, w_{3} x_{3}, \ldots\right)$, é um operador linear contínuo e mixing.

Para mais detalhes neste exemplo, ver [14, Corolário 8.3, pp. 217-218]. Para finalizar, observe que o backward shift $B_{w}$ no Exemplo 1.18 nada mais é que $B_{w_{n}}$ para $\left(w_{n}\right)_{n \in \mathbb{N}}=(w)_{n \in \mathbb{N}}$.

É imediato ver que um operador que é mixing é, obrigatoriamente, topologicamente transitivo e, portanto, hipercíclico (pelo Teorema da Transitividade de Birkhoff). No entanto, um resultado ainda melhor vale, como veremos no corolário deste lema:

Lema 1.23. Seja $T$ um operador que é mixing. Então $T \oplus T$ é mixing.

Demonstração: Como os produtos $U \times V$ de abertos $U, V \subseteq X$ formam uma base para topologia de $X \times X$, para mostrar que $T \oplus T$ é mixing podemos tomar, sem perda de generalização, abertos $U_{1} \times V_{1}$ e $U_{2} \times V_{2}$.

Sendo $T$ mixing, existem $k, l \in \mathbb{N}$ tal que $T^{n}\left(U_{1}\right) \cap V_{1} \neq \emptyset$, para todo $n \geq k$, e $T^{m}\left(U_{2}\right) \cap V_{2} \neq \emptyset$, para todo $m \geq l$. Tomando $N=\max \{k, l\}$, então vale que $(T \oplus T)^{n}\left(U_{1} \times U_{2}\right) \cap\left(V_{1} \times V_{2}\right) \neq \emptyset$, para todo $n \geq N$. Logo, $T \oplus T$ é mixing, como desejado.

\footnotetext{
${ }^{7}$ Isto é, espaços de Banach de dimensão finita não admitem operadores hipercíclicos.
} 
Corolário 1.24. Seja $T$ um operador que é mixing. Então $T$ satisfaz o Critério de Hiperciclicidade.

DemonstraçÃo: Pelo Lema anterior, temos que $T \oplus T$ é mixing. Logo, tendo em vista o que comentamos anteriormente, temos que $T \oplus T$ é hipercíclico. Pelo Teorema 1.20, segue que $T$ satisfaz o HC.

Vejamos mais um conceito que será bastante útil nos próximos capítulos:

Definição 1.25. Sejam $X, Y$ espaços de Banach e $T \in \mathcal{B}(X)$. Dizemos que $T$ é quasi-conjugado (via $\phi$ ) a um $S \in \mathcal{B}(Y)$ se existe $\phi: X \rightarrow Y$ contínua e com imagem densa tal que

$$
\phi \circ T=S \circ \phi
$$

Se, além disso, $\phi$ for linear, então dizemos que $T$ é linearmente quasi-conjugado (via $\phi$ ) a $S$.

Observe que existe uma sutil diferença entre $T$ ser quasi-conjugado a $S$ e $S$ ser quasi-conjugado a $T$. A saber, no primeiro caso a igualdade é $\phi \circ T=S \circ \phi$; já no segundo, a igualdade é $\phi \circ S=T \circ \phi$. Ainda, vale ressaltar que se $\phi \circ T=S \circ \phi$, então vale que $\phi \circ T^{m}=S^{m} \circ \phi$, para todo $m \in \mathbb{N}$.

Vejamos na próxima proposição como mixing e hiperciclicidade se "comportam bem" sob as quasi-conjugações:

Proposição 1.26. Sejam $X, Y$ espaços de Banach, $T \in \mathcal{B}(X)$ e $S \in \mathcal{B}(Y)$. Suponha que $T$ seja quasi-conjugado a $S$ (via $\phi$ ). Logo:

(a) se $T$ é hipercíclico, então $S$ é hipercíclico. Ainda, se $x \in X$ é um vetor hipercíclico para $T$, então $\phi(x)$ é um vetor hipercíclico para $S$.

(b) se T satisfaz o Critério de Hiperciclicidade, então $S$ também o satisfaz.

(c) se T é mixing, então $S$ também o é.

DemonstraÇÃo: (a) Seja $x$ um vetor hipercíclico para $T$. Se $U$ é um aberto de $Y$, então $\phi^{-1}(U)$ é aberto em $X$ (pois $\phi$ é contínua) e é não-vazio (pois $\phi$ tem imagem densa). Portanto, existe $n \in \mathbb{N}$ tal que $T^{n}(x) \in \phi^{-1}(U)$. Ou seja, $\phi\left(T^{n}(x)\right) \in U$, e como $\phi \circ T^{n}=S^{n} \circ \phi$ (via quasi-conjugação), segue que $S^{n}(\phi(x)) \in U$. Logo, orb $(\phi(x), S)$ é denso em $Y$, o que mostra que $S$ é hipercíclico com vetor hipercíclico $\phi(x)$.

(b) Como $T$ é quasi-conjugado a $S$, não é dificil ver que $T \oplus T$ é quasi-conjugado a $S \oplus S$. Como $T$ satisfaz o Critério, pelo Teorema 1.20, temos que $T \oplus T$ é hipercíclico. Daí, pelo item anterior, segue que $S \oplus S$ é hipercíclico, de onde concluímos (usando novamente o Teorema 1.20) que $S$ satisfaz o Critério.

(c) Sejam $U, V \subseteq Y$ abertos não-vazios. Como $\phi$ tem imagem densa, então $\phi^{-1}(U)$ e $\phi^{-1}(V)$ são abertos não-vazios em $X$. Como $T$ é mixing, então existe $k \in \mathbb{N}$ tal que $T^{n}\left(\phi^{-1}(U)\right) \cap \phi^{-1}(V) \neq \emptyset$, para todo $n \geq k$. 
Fixe $m \in \mathbb{N}$ tal que $m \geq k$. Então existe $y \in \phi^{-1}(U)$ tal que $T^{m} y \in \phi^{-1}(V)$. Como $y \in \phi^{-1}(U)$, então é claro que $\phi(y) \in U$. Ainda, como $T^{m} y \in \phi^{-1}(V)$, então $\phi\left(T^{m} y\right) \in V$. Como $\phi \circ T^{m}=S^{m} \circ \phi$, então segue que $S^{m}(\phi(y)) \in V$.

Portanto, temos que $\phi(y) \in U$ e $S^{m}(\phi(y)) \in V$. Logo, $S^{m}(U) \cap V \neq \emptyset$. Como $m \geq k$ é qualquer, então segue que $S$ é mixing, como desejado.

Chegamos, enfim, ao teorema que trata da existência de operadores hipercíclicos. Levando em conta o que foi dito no fim da seção anterior, tal teorema nos permite concluir que "ter dimensão infinita" e separabilidade são condições necessárias e suficientes para que exista um operador hipercíclico em espaços de Banach.

O teorema, para espaços de Banach, é devido à Ansari e Bernal. Ambos chegaram a este resultado de maneira independente. No entanto, acabaram por utilizar técnicas parecidas - a saber, o resultado de Ovsepian e Pełczyński (Teorema 1.8). Uma vez que existe uma generalização de tal resultado para espaços de Fréchet (ver [14, Lema 8.8, p. 220]), então é claro que o teorema também é válido para estes espaços (ver [14, Teorema 8.9, pp. 220-221]), indo de encontro com o que falamos após a definição de hiperciclicidade: apesar de apresentarmos os resultados em espaços de Banach, muitos deles ainda valem em outros espaços.

A demonstração que daremos é baseada na demonstração dada pelos autores da generalização para espaços de Fréchet: Bonet e Peris.

Teorema 1.27 (Ansari-Bernal). Todo espaço de Banach separável de dimensão infinita admite um operador que é mixing.

Demonstração: Seja $X$ um espaço de Banach separável de dimensão infinita. Assim, existe $\left\{x_{n}\right\}_{n=1}^{\infty} \subseteq X$ e $\left\{x_{n}^{*}\right\}_{n=1}^{\infty} \subseteq X^{*}$ satisfazendo as propriedades $(a),(b),(c)$ e $(d)$ do Teorema 1.8. Seja $T: X \rightarrow X$ dado por

$$
T x=x+\sum_{n=1}^{\infty} 2^{-n} x_{n+1}^{*}(x) x_{n}
$$

As propriedades do Teorema 1.8 implicam que $T$ está bem definido e é um operador linear limitado em $X$. Seja agora $S: \ell_{1} \rightarrow \ell_{1}$ dado por

$$
S\left(\left(\alpha_{n}\right)_{n \in \mathbb{N}}\right)=\left(\alpha_{1}+\frac{1}{2} \alpha_{2}, \alpha_{2}+\frac{1}{2^{2}} \alpha_{3}, \alpha_{3}+\frac{1}{2^{3}} \alpha_{4}, \ldots\right)
$$

Observe que $S$ é da forma $I+B_{w_{n}}, \operatorname{com} w_{n}=1 / 2^{n-1}$. Como sup $\left|w_{n}\right|<\infty$, então pelo Exemplo 1.22 temos que $S$ é mixing.

Seja $\phi: \ell_{1} \rightarrow X$ dado por $\phi\left(\left(\alpha_{n}\right)_{n \in \mathbb{N}}\right)=\sum_{n=1}^{\infty} \alpha_{n} x_{n}$. Note que $\phi$ é contínua e que tem imagem 
densa, tendo em vista o item (b) do Teorema 1.8. Agora:

$$
\begin{aligned}
T \circ \phi\left(\left(\alpha_{n}\right)_{n \in \mathbb{N}}\right) & =T\left(\sum_{n=1}^{\infty} \alpha_{n} x_{n}\right)=\sum_{n=1}^{\infty} \alpha_{n} T\left(x_{n}\right)=\alpha_{1} x_{1}+\sum_{n=2}^{\infty} \alpha_{n}\left(x_{n}+2^{-(n-1)} x_{n-1}\right) \\
& =\alpha_{1} x_{1}+\sum_{n=2}^{\infty}\left(\alpha_{n} x_{n}+\alpha_{n} 2^{-(n-1)} x_{n-1}\right)=\alpha_{1} x_{1}+\sum_{n=2}^{\infty} \alpha_{n} x_{n}+\sum_{n=2}^{\infty} \alpha_{n} 2^{-(n-1)} x_{n-1} \\
& =\sum_{n=1}^{\infty} \alpha_{n} x_{n}+\sum_{n=1}^{\infty} \alpha_{n+1} 2^{-n} x_{n}=\phi\left(\left(\alpha_{n}\right)_{n \in \mathbb{N}}\right)+\phi\left(\left(2^{-n} \alpha_{n+1}\right)_{n \in \mathbb{N}}\right) \\
& =\phi\left(\left(\alpha_{n}+2^{-n} \alpha_{n+1}\right)_{n \in \mathbb{N}}\right)=\phi \circ S\left(\left(\alpha_{n}\right)_{n \in \mathbb{N}}\right)
\end{aligned}
$$

Logo, $T \circ \phi=\phi \circ S$. Portanto, $S$ é quasi-conjugado a $T$. Assim, pela Proposição 1.26, segue que $T$ é mixing.

Observe que o operador $K(x)=\sum_{n=1}^{\infty} 2^{-n} x_{n+1}^{*}(x) x_{n}$ é o limite uniforme de operadores contínuos de posto finito 8 Como todo operador contínuo de posto finito é compacto e o limite (quando existir) de operadores compactos é compacto, segue que $K$ é compacto.9 Portanto, $T$ é da forma $I+K$, com $K$ compacto. Como $T$ satisfaz o Critério (vide o Corolário 1.24), então pelo Corolário 1.13 segue que $T$ é invertível.

Dessa forma, obtemos o seguinte corolário do teorema anterior:

Corolário 1.28. Todo espaço de Banach separável e de dimensão infinita admite um operador $T=$ $I+K$ (com $K$ compacto) invertivel que é mixing e, portanto, satisfaz o Critério de Hiperciclicidade.

Optamos por destacar tal resultado para facilitar futuras referências, uma vez que, ao contrário do Teorema 1.27, ele nos diz explicitamente que em todo espaço de Banach separável e de dimensão infinita existe um operador que é uma perturbação compacta da identidade (isto é, é da forma $I+K$, com $K$ compacto), é invertível e satisfaz o Critério de Hiperciclicidade.

Uma vez que mostramos a existência de operadores hipercíclicos, vamos apresentar na próxima seção mais alguns resultados em hiperciclicidade que serão úteis no restante do trabalho.

\subsection{Outros Resultados}

Seja $T: \mathbb{R}^{2} \rightarrow \mathbb{R}^{2}$ dado por $T(x, y)=(-y,-x)$. É fácil ver que $(1,0)$ é um vetor cíclico para $T$, assim como $(0,1)$. Agora, note que $T^{2}=I$. É claro que $T^{2}$ não é um operador cíclico. Este é, portanto, um exemplo de um operador cíclico tal que suas potências não o são.

Um dos primeiros resultados significativos na teoria dos operadores hipercíclicos foi obtido por Ansari em 1995. Ansari provou que o fenômeno descrito acima não acontece com operadores hipercíclicos: um operador é hipercíclico se, e somente se, todas as suas potências também o são.

\footnotetext{
${ }^{8}$ De fato, defina $K_{j}(x)=\sum_{n=1}^{j} 2^{-n} x_{n+1}^{*}(x) x_{n}$. Não é difícil ver que cada $K_{j}$ tem posto finito. Usando o fato de que, para todo $n \in \mathbb{N},\left\|x_{n}\right\|=1$, e $\sup _{n \in \mathbb{N}}\left\|x_{n}^{*}\right\|<\infty$, também não é difícil mostrar que a sequência dos $K_{j}$ converge uniformemente para $K$.

${ }^{9}$ Para uma referência em ambos os resultados ver, respectivamente, Proposição 3.4.3 e Proposição 3.4.8 de [22].
} 
Teorema 1.29 (Ansari). Seja X um espaço de Banach separável de dimensão infinita e tomemos $T \in \mathcal{B}(X)$. Se $T$ é um operador hipercíclico, então $T^{k}$ é um operador hipercíclico, para todo $k>1$. Além disso, temos que

$$
H(T)=H\left(T^{k}\right)
$$

para todo $k>1$.

DemonstraçÃo: Uma demonstração deste teorema pode ser encontrada em [14, Teorema 6.2, pp.162-163].

Como comentamos no início da seção, é claro que se $T^{k}$ é um operador hipercíclico (para qualquer $k>1$ ), então $T$ é um operador hipercíclico (uma vez que orb $\left(x, T^{k}\right) \subseteq \operatorname{orb}(x, T)$ ). $\mathrm{O}$ teorema acima diz respeito, portanto, à direção contrária de tal relação.

Tal propriedade dos operadores hipercíclicos é bastante útil e será utilizada em outros resultados, como veremos nos próximos capítulos. Devido a sua grande importância, nos referiremos muitas vezes ao teorema acima apenas por "Teorema de Ansari" ao invés da numeração dada.

O próximo resultado mostra algumas propriedades algébricas dos operadores hipercíclicos. A primeira propriedade, como pode-se ver na demonstração, é uma consequência do Teorema de Ansari:

Proposição 1.30. Seja $X$ um espaço de Banach separável de dimensão infinita e tomemos $T \in$ $\mathcal{B}(X)$. Se T é hipercíclico, então:

(1) $-T$ é hipercíclico.

(2) se $T$ é invertivel, então $T^{-1}$ é hipercíclico.

(3) se $\lambda \in \mathbb{K}$ é tal que $|\lambda|=1$, então $\lambda T$ é hipercíclico.

Demonstração:

(1) Segue do Teorema de Ansari (Teorema 1.29), visto que $(-T)^{2}=T^{2}$.

(2) Segue do Teorema da Transitividade de Birkhoff (Teorema 1.17), uma vez que $T^{n}(U) \cap V \neq \emptyset$ é equivalente a $U \cap T^{-n}(V) \neq \emptyset$, onde $\left(T^{-1}\right)^{n}=T^{-n}$.

(3) Tal resultado é conhecido como Teorema de León-Muller, vide [14, Teorema 6.7, p. 167].

Segue agora dois resultados envolvendo operadores hipercíclicos e polinômios:

Teorema 1.31 (Bourdon). Seja $T$ um operador hipercíclico e $p \in \mathbb{K}[x]$ um polinômio não-nulo. Então o operador $p(T)$ tem imagem densa.

Demonstração: Ver [14, Teorema 2.54, p.53] 
Corolário 1.32. Seja $T$ um operador hipercíclico e $p$ um polinômio não-nulo. Se $x$ é um vetor hipercíclico para $T$, então $p(T) x$ é um vetor hipercíclico para $T$.

Demonstração: Denote $y=p(T) x$. Observe que $T^{n} y=T^{n}(p(T) x)=p(T)\left(T^{n} x\right)$. Assim, $\operatorname{orb}(y, T)=p(T) \operatorname{orb}(x, T)$. Logo, sendo orb $(x, T)$ denso em $X$ (uma vez que $x$ é um vetor hipercíclico), para que orb $(y, T)$ também seja denso em $X$ basta que $p(T)$ tenha imagem densa. Tendo em vista o teorema anterior, temos o desejado.

Como consequência do último teorema e seu corolário, obtemos o próximo resultado:

Teorema 1.33 (Herrero-Bourdon). Seja $X$ um espaço de Banach separável. Se $T \in \mathcal{B}(X)$ é um operador hipercíclico, então $X$ admite um subespaço denso T-invariante consistindo, com exceção do zero, de vetores hipercíclicos.

DemonstraÇÃo: Seja $x$ um vetor hipercíclico para $T$. Observamos que

$$
M=\{p(T) x: p \text { é um polinômio }\}=\operatorname{span} \operatorname{orb}(x, T)
$$

é claramente um subespaço $T$-invariante. Ainda, é claro que $M$ é denso, uma vez que orb $(x, T) \subseteq M$ e, sendo $x$ um vetor hipercíclico, tal órbita é densa em $X$. Restaria mostrar, então, que todo $y \in M$ não-nulo é um vetor hipercíclico para $T$ : tal fato segue imediatamente do corolário anterior.

Para finalizar, temos o seguinte lema:

Lema 1.34. Se $x$ é um vetor hipercíclico para $T$, então orb $(x, T)$ é um conjunto linearmente independente.

Demonstração: Ver [14, Proposição 2.60, pp.55-56] 


\section{Capítulo 2}

\section{Sub-Hiperciclicidade}

Como mencionado na introdução, o conceito de sub-hiperciclicidade foi definido apenas em 2011, por Madore e Martínez-Avendaño. Dada a óbvia relação entre sub-hiperciclicidade e hiperciclicidade, imediatamente surgem algumas perguntas. Por exemplo, será que todo operador hipercíclico é sub-hipercíclico? Quais resultados para operadores hipercíclicos (em especial aqueles que apresentamos no capítulo anterior) valem para operadores sub-hipercíclicos?

A primeira pergunta foi respondida por Barmeni, Kadets e Kiliçman em [5]. Apesar da segunda pergunta ser naturalmente mais complexa, vamos tentar respondê-la neste capítulo. É claro que não será possível adaptar todos os resultados apresentados no capítulo anterior (e outros resultados conhecidos em hiperciclicidade apresentados em [14], por exemplo), uma vez que eles possuem diferentes graus de complexidade: mostrar que o Lema 1.34 vale para operadores sub-hipercíclicos é muito mais fácil que adaptar e mostrar uma versão sub-hipercíclica do Teorema de Ansari.

Procederemos então da seguinte maneira: na primeira seção vamos definir precisamente o conceito de sub-hiperciclicidade, dar alguns exemplos, levantar alguns problemas relevantes e demonstrar uma versão do Lema 1.34 para operadores sub-hipercíclicos (o Lema 2.9). Na segunda seção, vamos explorar a relação de operadores hipercíclicos com subespaços e sub-hiperciclicidade. Vamos mostrar que, dado um subespaço fechado não-trivial $M$, existe um operador $M$-hipercíclico que satisfaz Critério de Hiperciclicidade (este é o Teorema 2.17).

$\mathrm{Na}$ terceira seção vamos analisar como sub-hiperciclicidade e quasi-conjugações se relacionam, na tentativa de de adaptar e generalizar a Proposição 1.26. Já na seção seguinte mostraremos uma versão do Teorema de Ansari-Bernal (Teorema 1.27) para operadores sub-hipercíclicos (o Teorema 2.31). Para finalizar o capítulo, vamos generalizar um resultado conhecido de operadores hipercíclicos para operadores sub-hipercíclicos: vamos mostrar que o conjunto dos operadores subhipercíclicos é denso em $\mathcal{B}(X)$ quando munido da topologia da convergência pontual $\left.\right|^{1}$

\subsection{Definição, Exemplos e Resultados Gerais}

Vamos começar esta seção com a definição de sub-hiperciclicidade:

Definição 2.1 (Definição 2.1 de [18]). Seja X um espaço de Banach de dimensão infinita e $T \in$ $\mathcal{B}(X)$. Dizemos que $T$ é sub-hipercíclico se existem um $x \in X$ e um subespaço fechado de

\footnotetext{
${ }^{1}$ Veremos que este é o melhor resultado que podemos obter, uma vez que não é possível que o conjunto dos operadores sub-hipercíclicos seja denso em $\mathcal{B}(X)$ quando este estiver munido da topologia usual (uma consequência direta do Lema 2.11.
} 
dimensão infinita não-trivia $M$ tal que orb $(x, T) \cap M$ é denso em $M$. Neste caso, também dizemos que $T$ é $M$-hipercíclico ou sub-hipercíclico para $M$ e que $x$ é um vetor $M$-hipercíclico para $\mathbf{T} 3$

Veremos, na Proposição 2.8, que podemos tirar a exigência de que $M$ tenha dimensão infinita (uma vez que se $T$ for $M$-hipercíclico, então $M$ fatalmente terá dimensão infinita).

Vamos agora apresentar dois exemplos. O primeiro, embora simples, responde negativamente a pergunta natural: será que todo operador sub-hipercíclico é hipercíclico?

Exemplo 2.2 (Exemplo 2.2 de [18]). Seja $T$ um operador hipercíclico em um espaço separável $X$ e considere o subespaço $M=X \oplus\{0\}$ de $X \oplus X$. É claro que $M$ é fechado em $X \oplus X$ e o operador $T \oplus I$ (onde $I$ é a identidade) é sub-hipercíclico para $M$ (uma vez que $T$ é hipercíclico em $X$ ). No entanto, é claro que $T \oplus I$ não é hipercíclico em $X \oplus X$.

O segundo exemplo, um pouco menos trivial, além de nos mostrar que nem todo operador subhipercíclico é hipercíclico, também mostra que, ao contrário de hiperciclicidade, sub-hiperciclicidade pode sim acontecer em espaços não-separáveis:

Exemplo 2.3. Seja $w \in \mathbb{R}$ tal que $|w|>1$. Considere $B_{w}$ o backward shift em $\ell_{\infty}$ dado por $B_{w}\left(x_{1}, x_{2}, x_{3}, \ldots\right)=w \cdot\left(x_{2}, x_{3}, x_{4}, \ldots\right)$. Vimos, no Exemplo 1.18 , que $B_{w}$ é um operador hipercíclico em $c_{0}$. Agora, identificando naturalmente $c_{0}$ como um subespaço de $\ell_{\infty}$, temos que $B_{w}$ é $c_{0^{-}}$ hipercíclico em $\ell_{\infty}$ (e é, obviamente, não-hipercíclico, visto que $\ell_{\infty}$ não é separável).

Note que o último exemplo nos mostra também que, se $M \subsetneq X$ é um subespaço tal que existe um $T \in \mathcal{B}(X)$ com $T$ sendo $M$-hipercíclico, então não necessariamente $M$ é complementado em $X$, uma vez que, no exemplo acima, temos que $B_{w} \in \mathcal{B}\left(\ell_{\infty}\right)$ é $c_{0}$-hipercíclico mas $c_{0}$ não é complementado em $\ell_{\infty}$ (ver [2, Teorema 2.5.5, p. 46]).

Em ambos os exemplos foi razoavelmente simples de se mostrar que ambos os operadores são subhipercíclicos. Isto aconteceu justamente pelo fato de estarmos considerando operadores hipercíclicos em subespaços invariantes, como vamos destacar na observação a seguir:

Observação 2.4. Sejam $T \in \mathcal{B}(X)$ um operador qualquer e $M \subseteq X$ um subespaço fechado separável de dimensão infinita. Suponha que $T$ seja $M$-invariante (isto é, $T(M) \subseteq M$ ). Logo, o operador $\left.T\right|_{M}: M \rightarrow M$ está bem definido. Assim, não é difícil perceber então que $T$ é $M$ hipercíclico se, e somente se, $\left.T\right|_{M}$ é hipercíclico (como um operador de $\mathcal{B}(M)$ ), considerando $M$ como um espaço de Banach).

\footnotetext{
${ }^{2}$ Isto é, $M \neq X$ e $M \neq\{0\}$.

${ }^{3}$ Seria natural tentar definir operadores sub-hipercíclicos como sendo operadores $T$ tais que existe um $x \in X$ com orb $(x, T) \subseteq M$ sendo denso em $M$. No entanto, é fácil de se observar que, se fosse esse o caso, então todo operador $M$-hipercíclico seria também $M$-invariante; portanto, $\left.T\right|_{M}$ estaria bem definido e seria um operador hipercíclico. Logo, todo o conceito de sub-hiperciclicidade se reduziria a um caso particular de hiperciclicidade, a saber, o de operadores hipercíclicos invariantes em subespaços.
} 
Feita essa observação, podemos dizer que, sob o ponto de vista de subespaços invariantes, subhiperciclicidade é equivalente a hiperciclicidade. Por esse motivo, ao longo do texto tomaremos o cuidado de evitar estudar sub-hiperciclicidade em tais espaços uma vez que estaríamos estudando, de um jeito "disfarçado", hiperciclicidade - e este não é o objetivo principal deste trabalho.

Tomando um pouco de liberdade, podemos dizer que um operador hipercíclico nada mais é que um operador $X$-hipercíclico. Apesar desse caso não ser tão interessante quando estamos estudando os subespaços de $X$ e os operadores sub-hipercíclicos, tal liberdade será tomada algumas vezes no restante do texto. Vale frisar que um operador $X$-hipercíclico ainda é interessante (uma vez que, neste caso, ele se mostra hipercíclico).

Antes de prosseguirmos, vejamos mais um exemplo. Nele vamos mostrar que o fato de $M \subseteq$ $\overline{\operatorname{orb}(z, T)}$ não implica que $T$ seja $M$-hipercíclico com vetor $z$ :

Exemplo 2.5 (Exemplo 3 de [16]). Seja $H$ um espaço de Hilbert separável. Usando o Teorema 8.13 de [14], tome $T: H \rightarrow H$ um operador hipercíclico tal que $T x=x$, para todo $x \in H_{1}$, onde $H_{1}$ é um subespaço fechado de dimensão infinita.

Seja agora $z$ um vetor hipercíclico para $T$. Considere $M=H_{1} \oplus \operatorname{span}\{z\}$. É claro que $M$ é fechado e tem dimensão infinita. Ainda, como $z$ é um vetor hipercíclico, então $M \subseteq \overline{\operatorname{orb}(z, T)}=H$. No entanto, vamos mostrar que orb $(z, T) \cap M=\{z\}$. Isto provará que $z$ não é um vetor $M$ hipercíclico para $T$.

Seja $y \in \operatorname{orb}(z, T) \cap M$. Então $y=T^{n} z=x+\lambda z$, com $x \in H_{1}$. Segue que $\left(T^{n}-\lambda I\right) z=x$, donde obtemos um absurdo. De fato, observe que o lado direito da igualdade é um vetor fixo para $T$ (isto é, $T^{n} x=x$, para todo $n \in \mathbb{N}$ ). Por outro lado, temos que $\left(T^{n}-\lambda I\right) z$ é um vetor hipercíclico para $T$ (vide o Corolário 1.32 .

Agora, ainda questionando a relação citada anteriormente, será que todo operador hipercíclico também é sub-hipercíclico, para algum $M$ não-trivial? Essa pergunta tem uma resposta positiva, sendo respondida por Bamerni, Kadets e Kiliçman. Tal resposta veio como um corolário de um resultado mais geral demonstrado pelos mesmos três autores, como veremos a seguir:

Teorema 2.6 (Teorema 2.1 de [5]). Se A é um subconjunto denso de um espaço de Banach $X$, então existe um subespaço fechado de dimensão infinita $M($ com $M \neq X)$ tal que $A \cap M$ é denso em $M$.

Apesar de ser um corolário imediato do teorema anterior, denotaremos como teorema o resultado a seguir devido à sua importância dentro do tema:

Teorema 2.7 (Corolário 2.4 de [5]). Todo operador hipercíclico é sub-hipercíclico.

Com esse resultado, muitas questões foram levantadas pelos autores em [5]. Destacamos:

Problema 1 (Pergunta 1 de [5]). Se $T$ é sub-hipercíclico para $M_{1}$ e $T^{-1}$ é sub-hipercíclico para $M_{2}$, qual a relação entre $M_{1}$ e $M_{2}$ ? 
Apenas para ficar claro, tal problema acima "faz sentido" pois, se $T$ é invertível e hipercíclico, então $T^{-1}$ também é hipercíclico (vide a Proposição 1.30). Logo, pelo teorema anterior, ambos são sub-hipercíclicos e, portanto, é interessante descobrir se existe uma relação entre os subespaços para os quais $T$ e $T^{-1}$ são sub-hipercíclicos.

Problema 2 (Pergunta 2 de [5]). Se $T$ é sub-hipercíclico para $M_{1}$ e $\lambda T$ é sub-hipercíclico para $M_{2}$, qual a relação entre $M_{1}$ e $M_{2}$ ?

No espírito do problema (e do parágrafo) anterior, tal pergunta faz sentido uma vez que sabemos que, se $T$ é hipercíclico, então $\lambda T$ é hipercíclico, para todo $\lambda$ tal que $|\lambda|=1$ (vide a Proposição 1.30 .

Problema 3 (Pergunta 3 de [5]). Se $T^{n}$ é sub-hipercíclico para $M_{n}$, para $n \geq 1$, qual a relação entre os $M_{n}$ ?

De novo, esta pergunta faz sentido uma vez que lembramos do Teorema de Ansari (Teorema 1.29). Vale notar que orb $\left(x, T^{n}\right) \subseteq \operatorname{orb}(x, T)$ e, portanto, $T$ será $M_{n}$-hipercíclico, para todo $n \in \mathbb{N}$. No entanto, como veremos no Exemplo 2.22, o fato de $T$ ser sub-hipercíclico para dois subespaços distintos em geral não é uma condição que nos permita obter uma relação de isomorfismo entre os subespaços (e veremos também em tal exemplo que também podemos ter $T$ sub-hipercíclico para $M, N$ e $M \cap N=\{0\})$.

Voltaremos a estes problemas mais adiante. Por ora, apresentaremos mais alguns resultados relativos à sub-hiperciclicidade.

Como era de se esperar (devido à clara relação entre hiperciclicidade e sub-hiperciclicidade), é natural que tomemos os resultados de hiperciclicidade e tentemos fazer resultados "análogos" para sub-hiperciclicidade. Vamos apresentar alguns destes resultados agora. O primeiro resultado mostra que o subespaço $M$ precisa ter dimensão infinita para que exista sub-hiperciclicidade:

Proposição 2.8 (Teorema 4.9 e Teorema 4.10 de [18]). Sejam $X$ um espaço de Banach e $M \subsetneq X$ um subespaço. Se $T \in \mathcal{B}(X)$ é $M$-hipercíclico, então $M$ tem dimensão infinita $\bigsqcup^{4}$

É claro que, se $M$ é um subespaço não-trivial de dimensão infinita, então $X$ também tem dimensão infinita. Dito isso, esta proposição nos mostra que, assim como hiperciclicidade, ter dimensão infinita é uma condição necessária para que um subespaço $M$ possa ser $M$-hipercíclico 5 Como separabilidade também é uma condição necessária para o subespaço $M$ (uma consequência imediata da Definição 2.1), podemos então concluir que estas duas condições são necessárias tanto para a existência de operadores sub-hipercíclicos quanto para a existência de operadores hipercíclicos (no primeiro caso, tais exigências são feitas sobre o subespaço; já no segundo caso, sobre o espaço inteiro).

Como vimos no Corolário 1.28 , estas condições (ter dimensão infinita e separabilidade) também são suficientes para hiperciclicidade. No contexto de verificar similaridades entre hiperciclicidade

\footnotetext{
${ }^{4}$ Vale salientar que o enunciado original deste resultado é para espaços de Hilbert. No entanto, uma verificação da demonstração feita em [18 nos permite concluir que tal resultado também vale para espaços de Banach (ver, por exemplo, a citação feita a este resultado em [24, Teorema 1.1]).

${ }^{5}$ Estamos cometendo um pequeno abuso de linguagem aqui; queremos dizer, com esta expressão, que tal condição é necessária para que exista um operador $T \in \mathcal{B}(X)$ tal que $T$ é $M$-hipercíclico.
} 
entre sub-hiperciclicidade, seriam tais condições, quando feitas sobre $M$, também suficientes? A resposta é positiva e será mostrada ainda neste capítulo: em um primeiro momento, supondo que o espaço $X$ é separável, a resposta será dada pelo Teorema 2.17. Para o caso em que $X$ não for separável, a resposta será dada pelo Teorema 2.32 .

O próximo resultado é o análogo do Lema 1.34 para sub-hiperciclicidade.

Lema 2.9. Seja $T$ um operador $M$-hipercíclico. Se x é um vetor $M$-hipercíclico para $T$, então orb $(x, T)$ é um conjunto linearmente independente.

DemonstraçÃo: Suponha que orb $(x, T)$ seja linearmente dependente. Temos então que existem $k \in \mathbb{N}$ e escalares $a_{1}, \ldots, a_{k-1}$ não todos nulos tais que

$$
T^{k}(x)=\sum_{j=1}^{k-1} a_{j} T^{j} x
$$

Dessa forma, temos que se $A=\operatorname{span}\left\{T^{j}(x): 1 \leq j \leq k-1\right\}$, então $T^{k}(x) \in A$ e portanto

$$
T^{k+1}(x)=T\left(T^{k} x\right)=T\left(\sum_{j=1}^{k-1} a_{j} T^{j} x\right)=\sum_{j=1}^{k-1} a_{j} T^{j+1} x \in A
$$

uma vez que $A$ é um subespaço. Indutivamente, segue que $T^{j} x \in A$, para todo $j \in \mathbb{N}$. Dessa forma, temos que span orb $(x, T)=A$.

Sendo $T$ um operador $M$-hipercíclico, então orb $(x, T) \cap M=\left\{T^{n_{1}} x, T^{n_{2}} x, \ldots, T^{n_{k}} x, \ldots\right\}$. Seja agora $N=\operatorname{span}\left\{T^{n_{j}}(x): j \in \mathbb{N}\right\}$. Como orb $(x, T) \cap M \subseteq \operatorname{orb}(x, T)$, então $N=\operatorname{span}[\operatorname{orb}(x, T) \cap$ $M] \subseteq \operatorname{span} \operatorname{orb}(x, T)=A$. Portanto, como $\operatorname{dim} A<\infty$, segue que $\operatorname{dim} N<\infty$ e $N$ é fechado.

Portanto, como orb $(x, T) \cap M \subseteq N$, segue que $M=\overline{\operatorname{orb}(x, T) \cap M} \subseteq \bar{N}=N$, um absurdo pois $\operatorname{dim} M=\infty$ (vide a Proposição 2.8, uma vez que $T$ é $M$-hipercíclico) e $\operatorname{dim} N<\infty$.

Logo, orb $(x, T)$ é linearmente independente, como desejado.

Observe agora que faz sentido estudarmos sub-hiperciclicidade apenas em operadores que possuam um vetor $x$ tal que orb $(x, T)$ é linearmente independente pois, como vimos acima, tal condição se faz necessária. No entanto, veremos a seguir que ela não é suficiente:

Exemplo 2.10. Seja $F$ o forward shift em $\ell_{\infty}$, isto é, $F\left(x_{1}, x_{2}, x_{3}, \ldots\right)=\left(0, x_{1}, x_{2}, x_{3}, \ldots\right)$. Note que existe um $x \in \ell_{\infty}$ tal que orb $(x, F)$ é linearmente independente - a saber, $x=e_{1}$, onde $e_{1}=(1,0,0,0, \ldots)$. Daí, é claro que $F^{n} e_{1}=e_{n}$ e, portanto, orb $\left(e_{1}, F\right)=\left\{e_{1}, e_{2}, e_{3}, \ldots, e_{n}, \ldots\right\}$ é um conjunto linearmente independente.

Suponha agora que $F$ seja $M$-hipercíclico com vetor $x$. Tome $y \in M$ tal que $\|y\|>1+\|x\|$. Então, para todo $n \in \mathbb{N}$,

$$
\left\|F^{n} x-y\right\| \geq\|y\|-\left\|F^{n} x\right\|=\|y\|-\|x\|>1
$$

uma vez que $\left\|F^{n} x\right\|=\|x\|$, para todo $n \in \mathbb{N}$. 
Logo, fica claro que não podemos ter orb $(x, F) \cap M$ denso em $M$.

Podemos concluir o exemplo anterior de outra maneira: não é difícil perceber que $\left\|F^{n} x\right\| \leq\|x\|$. Esta desigualdade claramente mostra que orb $(x, F)$ é limitado e, com isso, é claro que orb $(x, F) \cap M$ não pode ser denso em $M$, como desejado.

Vamos definir, em um outro capítulo mais adiante, a noção de sub-ciclicidade e veremos, na Proposição 4.28, que ter uma órbita linearmente independente (e infinita) é uma condição suficiente para que um operador seja sub-cíclico, ao contrário do que acontece com sub-hiperciclicidade, como vimos no exemplo acima.

Não é difícil ver que, com o mesmo argumento do exemplo anterior, podemos mostrar que $F$ não é um operador hipercíclico. Além disso, não é difícil perceber que tal argumento também demonstra o seguinte lema:

Lema 2.11. Seja $T$ um operador $M$-hipercíclico. Então $\|T\|>1$.

Utilizando este mesmíssimo argumento, não é difícil verificar que o lema acima também vale para operadores hipercíclicos, indo de acordo com a nossa intenção de fazer resultados "análogos" para sub-hiperciclicidade.

Vimos, no Teorema 2.7, que todo operador hipercíclico é sub-hipercíclico. Respondida essa importante pergunta, podemos agora estudar a relação entre operadores hipercíclicos e subespaços de outras maneiras. Como discutimos anteriormente, dado um subespaço $M \subsetneq X$, existe um operador hipercíclico tal que $T$ é sub-hipercíclico para $M$ ? E dados um operador hipercíclico $T$ e um subespaço $M$ qualquer, é possível que $T$ seja $M$-hipercíclico?6

A primeira pergunta é respondida no Teorema 2.17, apresentado na próxima seção. Já para a segunda pergunta, observe que a resposta é negativa: basta considerar, por exemplo, o operador $T$ e o subespaço $H_{1}$ descritos no Exemplo 2.5 7 Apesar dessa resposta negativa, Subrahmonian Moothathu mostrou em [26] um resultado interessante que, considerando o exemplo que acabamos de descrever, é o melhor possível que podemos obter: dados $T$ hipercíclico e $M$, existe $R: X \rightarrow X$ operador linear invertível tal que $T$ é sub-hipercíclico para $R(M)$ (Teorema 2.16).

Veremos tais resultados na próxima seção.

\subsection{Operadores Hipercíclicos, Subespaços e Sub-Hiperciclicidade}

Como é evidente (pela numeração adotada), vamos primeiro apresentar o Teorema 2.16, uma vez que utilizamos tal resultado para demonstrar o Teorema 2.17. No entanto, para apresentar o Teorema 2.16, precisaremos de alguns resultados auxiliares e uma nova definição. Comecemos com um teorema:

\footnotetext{
${ }^{6}$ Estamos considerando neste parágrafo que $M$ é um subespaço fechado, separável, de dimensão infinita tal que $M \neq X$.

${ }^{7}$ De fato, lembremos que $H_{1}$ tem a propriedade de que se $h \in H_{1}$ então $T h=h$. Logo, se $y \in \operatorname{orb}(x, T) \cap H_{1}$, então $y=T^{n} x$ e como $y \in H_{1}, y=T^{n+k} x, k \in \mathbb{N}$. Portanto, orb $(x, T) \cap H_{1}=\{y\}$. Daí, é claro que orb $(x, T) \cap H_{1}$ não é denso em $H_{1}$.
} 
Teorema 2.12 (Subrahmonian Moothathu, [26]). Seja X um espaço de Banach separável de dimensão infinita e tome $J$ um conjunto enumerável. Para cada $j \in J$, sejam $A_{j}=\left\{a_{j, n}: n \in \mathbb{N}_{0}\right\}$ e $C_{j}=\left\{c_{j, n}: n \in \mathbb{N}_{0}\right\}$ conjuntos infinitos, enumeráveis e densos em $X$ tais que

(a) $A_{i} \cap A_{j}=\emptyset=C_{i} \cap C_{j}$, para quaisquer $i, j \in J$ distintos

(b) $\cup_{j \in J} A_{j} e \cup_{j \in J} C_{j}$ são conjuntos linearmente independentes em $X$.

Então para cada $\varepsilon>0$, existe $R \in \mathcal{B}(X)$ invertivel tal que $\|I-R\|<\varepsilon$ e $R\left(A_{j}\right)=C_{j}$, para todo $j \in J$. Ainda, se para um $j \in J$ fixo tivermos que $\left\|a_{j, 0}-c_{j, 0}\right\|<\varepsilon\left\|a_{j, 0}\right\|$, então $R$ pode ser escolhido com a propriedade adicional de que $R a_{j, 0}=c_{j, 0}$, para tal $j$ fixado.

DemonstraÇÃo: Ver [26, Teorema 1, p. 1184].

Note que este último teorema é uma generalização do Lema 1.3. A seguir, vejamos uma definição que será utilizada nos próximos resultados:

Definição 2.13 ([26]). Dizemos que $M \subseteq \mathbb{N}_{0}$ é um AP-set $]^{8}$ se existem $k, l \in \mathbb{N}$ tais que $k \mathbb{N}_{0}+l \subseteq$ $M$.

Observe que, pelo Teorema de Ansari (Teorema 1.29), temos que $T$ é hipercíclico se, e somente se, $T^{k}$ é hipercíclico; além disso, vale também que $H(T)=H\left(T^{k}\right)$. Isto demonstra o seguinte lema:

Lema 2.14 (Subrahmonian Moothathu, [26]). Seja $T$ um operador hipercíclico e tome $x \in H(T)$. Então $\left\{T^{n} x: n \in M\right\}$ é denso em $X$ para todo AP-set $M \subseteq \mathbb{N}_{0}$.

Por fim, o próximo lema tem um papel importante na demonstração do Teorema 2.16 .

Lema 2.15 (Subrahmonian Moothathu, [26]). Sejam X um espaço de Banach separável de dimensão infinita, $T \in \mathcal{B}(X)$ um operador hipercíclico, $x \in H(T)$ e $Y \neq X$ um subespaço fechado de dimensão infinita de $X$. Seja também $Q$ um conjunto enumerável. Para $q \in Q$, seja $Z_{q} \subseteq Y$ um conjunto enumerável tal que $\cup_{q \in Q} Z_{q}$ é vazio ou linearmente independente. Tome, para todo $q \in Q$, $M_{q} \subseteq \mathbb{N}_{0}$ AP-sets dois-a-dois disjuntos. Então para todo $\varepsilon>0$, existe $R \in \mathcal{B}(X)$ invertivel tal que $\|I-R\|<\varepsilon$, orb $(x, T) \cap R(Y)=\cup_{q \in Q} R\left(Z_{q}\right)$ e $\left\{n \in \mathbb{N}_{0}: T^{n} x \in R\left(Z_{q}\right)\right\} \subseteq M_{q}$ para todo $q \in Q$.

Demonstração: Ver [26, Corolário 1, p. 1187].

Apesar do próximo resultado não ser de nossa autoria, optamos por exibir a demonstração do mesmo pois, com base nessa demonstração, faremos algumas observações relacionadas ao Teorema $2.17^{9}$

\footnotetext{
${ }^{8}$ Nomenclatura oriunda do inglês, onde $A P$ significa "Arithmetic Progression".

${ }^{9}$ Lembrando que, como dissemos no início desta seção, na demonstração do Teorema 2.17 vamos utilizar o Teorema 2.16
} 
Teorema 2.16 (Subrahmonian Moothathu, [26]). Sejam X um espaço de Banach separável de dimensão infinita e $T: X \rightarrow X$ um operador hipercíclico. Então dado $Y \neq X$ um subespaço fechado de dimensão infinita de $X$, para todo $\varepsilon>0$, existe $R: X \rightarrow X$ operador linear invertivel tal que $\|I-R\|<\varepsilon$ e $T^{q}$ é sub-hipercíclico para $R(Y)$, para todo $q \in \mathbb{Z}^{+}$.

DemonstraÇÃo: Seguiremos a demonstração original. Para $q \in \mathbb{N}$, seja $M_{q}=\left\{2^{q} q n+2^{q-1}: n \in\right.$ $\mathbb{N}$ \} e observe que os $M_{q}$ são dois-a-dois disjuntos e AP-sets. Ainda, tome agora $Z_{q}$ subconjuntos densos em $Y$ dois-a-dois disjuntos tais que $\cup_{q \in \mathbb{N}} Z_{q}$ é linearmente independente. Observe que isso é possível pois $Y$ tem dimensão infinita.

Tome $x \in H(T)$. Pelo lema anterior, existe $R \in \mathcal{B}(X)$ invertível tal que orb $(x, T) \cap R(Y)=$ $\cup_{q \in \mathbb{N}} R\left(Z_{q}\right)$ e $\left\{n \in \mathbb{N}_{0}: T^{n} x \in R\left(Z_{q}\right)\right\} \subseteq M_{q}$ para todo $q \in \mathbb{N}$. Agora, fixe um $q \in \mathbb{N}$. Seja $m_{q}$ o menor elemento de $M_{q}$ tal que $v_{q}:=T^{m_{q}} x \in R\left(Z_{q}\right)$.

Observe agora que se $m_{q}:=2^{q} q k+2^{q-1}$, então o próximo elemento de $M_{q}$, que é $n_{q}:=$ $2^{q} q(k+1)+2^{q-1}$, é tal que $n_{q}-m_{q}=2^{q} q$. Portanto, temos que

$$
\left\{T^{m_{q}} x, T^{n_{q}} x\right\} \subseteq\left\{v_{q}, T^{q} v_{q}, T^{2 q} v_{q}, \ldots, T^{2^{q} q} v_{q}\right\} \subseteq \text { orb }\left(v_{q}, T^{q}\right)
$$

Portanto, como $\left\{n \in \mathbb{N}_{0}: T^{n} x \in R\left(Z_{q}\right)\right\} \subseteq M_{q}$ e orb $(x, T) \cap R(Y)=\cup_{q \in \mathbb{N}} R\left(Z_{q}\right)$, da inclusão em destaque acima segue que $R\left(Z_{q}\right) \subseteq$ orb $\left(v_{q}, T^{q}\right)$. Como $Z_{q} \subseteq Y$, então $R\left(Z_{q}\right) \subseteq R(Y)$, donde temos que

$$
R\left(Z_{q}\right) \subseteq \operatorname{orb}\left(v_{q}, T^{q}\right) \cap R(Y)
$$

Agora, como $R\left(Z_{q}\right)$ é denso em $R(Y)$ (uma vez que $Z_{q}$ é denso em $Y$ ), segue que orb $\left(v_{q}, T^{q}\right) \cap$ $R(Y)$ é denso em $R(Y)$, o que mostra que $T^{q}$ é sub-hipercíclico para $R(Y)$, como desejado.

É interessante observar que o teorema anterior aponta para uma direção na tentativa de resolver o Problema 3. será que se $T^{n}$ for $M_{n}$-hipercíclico, para todo $n \in \mathbb{N}$, então existe um isomorfismo $R_{m, n} \in \mathcal{B}(X)$ tal que $R\left(M_{m}\right)=M_{n}$ ? Lembramos que tal isomorfismo, em geral, pode não existir (como explicamos após tal problema e veremos no Exemplo 2.22). No entanto, o fato de estarmos trabalhando com potências do mesmo operador (e tendo em mente o Teorema de Ansari para operadores hipercíclicos) talvez torne a tarefa mais fácil.

Feitos estes questionamentos, podemos agora exibir o teorema que responde positivamente a pergunta levantada no fim da seção anterior para o caso em que $X$ é separável: dado um subespaço $M \subsetneq X$ fechado e de dimensão infinita, existe um operador hipercíclico tal que $T$ é sub-hipercíclico para $M$ ?

Teorema 2.17. Seja $X$ um espaço de Banach separável e de dimensão infinita. Seja $M \neq X$ um subespaço fechado de dimensão infinita. Então existe $T \in \mathcal{B}(X)$ invertível e que satisfaz o Critério de Hiperciclicidade tal que $T$ é sub-hipercíclico para $M$.

DemonstraÇão: Pelo Corolário 1.28, $X$ admite um operador hipercíclico invertível $S$ que satisfaz o Critério. Assim, pelo teorema anterior existe $R$ invertível tal que $S$ é sub-hipercíclico para $R(M)$; isto é, orb $(z, S) \cap R(M)$ é denso em $R(M)$, para algum $z \in X$. 
Considere $x:=R^{-1}(z)$. Como $R^{-1}$ é contínuo, $R^{-1}(\operatorname{orb}(z, S) \cap R(M))=R^{-1}(\operatorname{orb}(z, S)) \cap M$ é denso em $M$ (lembrando também que $R^{-1}$ é injetor). Agora,

$$
\begin{aligned}
R^{-1}(\operatorname{orb}(z, S)) & =R^{-1}\left(\left\{z, S z, S^{2} z, \ldots\right\}\right)=R^{-1}\left(\left\{R x, S R x, S^{2} R x, \ldots\right\}\right)= \\
& \left.=\left\{x, R^{-1} S R x, R^{-1} S^{2} R x, \ldots\right\}\right)=\operatorname{orb}(x, T)
\end{aligned}
$$

onde $T=R^{-1} \circ S \circ R$.

Logo, $T$ é sub-hipercíclico para $M$. Pela definição de $T$, é claro que $T$ é invertível (visto que $S$ é invertível). Para ver que $T$ é hipercíclico e que satisfaz o Critério, basta observar que $T \circ R^{-1}=R^{-1} \circ S$. Daí, como $R^{-1}$ é contínuo e com imagem densa, então $S$ é quasi-conjugado a $T$ (via $R^{-1}$ ). Logo, como $S$ satisfaz o Critério, pela Proposição 1.26 temos que $T$ satisfaz o Critério, como desejado.

Veremos no Teorema 2.32 que o resultado acima se estende a espaços de Banach não-separáveis (considerando $M \neq X$ um subespaço separável de dimensão infinita). Por ora, vejamos agora duas observações pertinentes ao teorema anterior:

Observação 2.18. Não foi deixado explícito na demonstração do teorema acima, mas temos que $x:=R^{-1} z$ (usando ainda a notação da demonstração) é tal que $x \in H(T)$.

Para podermos constatar isso, temos que voltar primeiro para a demonstração do Teorema 2.16 . Utilizando a notação da demonstração daquele teorema, temos que $v_{1}:=T^{m_{1}} x$ é o vetor $R(Y)$ hipercíclico para $T$. Lembrando do Teorema de Ansari (Teorema 1.29), temos que $T^{m_{1}} x \in H(T)$ (uma vez que tomamos $x \in H(T)$ na demonstração do Teorema 2.16.

Portanto, ao recorrermos ao Teorema 2.16 na demonstração do teorema anterior, podemos supor que $z$ é tal que $z \in H(S)$. Uma vez que $S$ é quasi-conjugado a $T$ via $R^{-1}$, então pela Proposição 1.26 temos que $R^{-1} z=x$ é um vetor hipercíclico para $T$.

Observação 2.19. Note que o operador $T$ obtido no Teorema 2.17 também é mixing. De fato, pelo Corolário 1.28, $S$ é mixing e como $S$ é quasi-conjugado com $T$, então segue que $T$ é mixing (vide a Proposição 1.26 ).

Continuando no tema dos resultados anteriores (isto é, operadores hipercíclicos e sua relação com subespaços), obtemos mais um resultado:

Teorema 2.20. Sejam T, $S$ dois operadores hipercíclicos. Então existem $M$ subespaço não-trivial de dimensão infinita e um operador linear invertível $A: X \rightarrow X$ tal que $T$ é $M$-hipercíclico e $S$ é $A(M)$-hipercíclico.

DemonstraÇÃo: Sejam $x$ um vetor hipercíclico para $T$ e $y$ um vetor hipercíclico para $S$. Denote $X_{0}=\operatorname{orb}(x, T)$ e $Y_{0}=\operatorname{orb}(y, S)$. Como ambos os conjuntos são linearmente independentes (vide 
o Lema 1.34 e densos em $X$, então pelo Lema 1.3 temos que existe $A: X \rightarrow X$ operador linear invertível tal que $A\left(X_{0}\right)=Y_{0}$.

Pelo Teorema 2.6 temos que existe um subespaço não-trivial de dimensão infinita $M$ tal que $X_{0} \cap M$ é denso em $M$. Denote agora $X_{1}:=X_{0} \cap M$. Temos que $A(M)=A\left(\overline{X_{1}}\right)=\overline{A\left(X_{1}\right)}$ (uma vez que $A$ é invertível). Como $A\left(X_{1}\right) \subseteq A(M)$ (pois $\left.X_{1} \subseteq M\right)$, segue então que $A\left(X_{1}\right)=A\left(X_{1}\right) \cap A(M)$ e, portanto, que $A(M)=\overline{A\left(X_{1}\right) \cap A(M)}$.

Para finalizar, observe que, como $X_{1} \subseteq X_{0}$, então $A\left(X_{1}\right) \subseteq A\left(X_{0}\right)=Y_{0}$. Portanto, segue que $A\left(X_{1}\right) \cap A(M) \subseteq Y_{0} \cap A(M) \subseteq A(M)$. Logo, temos que $A(M)=\overline{A\left(X_{1}\right) \cap A(M)} \subseteq \overline{Y_{0} \cap A(M)} \subseteq$ $A(M)$, o que mostra que $Y_{0} \cap A(M)$ é denso em $A(M)$, como desejado.

Com o teorema anterior em mente, é natural pensar: podemos aprimorar tal resultado? Ou seria tal enunciado o "melhor possível" de se obter (no mesmo espírito do Teorema 2.16)? Resumidamente, temos o seguinte problema:

Problema 4. Dados $T, S$ hipercíclicos, existe $M$ tal que $T$ e $S$ são $M$-hipercíclicos?

Uma vez que o operador $A$ obtido pelo Teorema 2.20 é obtido através de outro resultado (e também porque tal operador não é obtido com o subespaço $M$ em mente), vemos que não há muita margem para melhorar a demonstração feita de modo a termos $A=I$. Dessa forma, tentamos encontrar outros meios de atacar tal problema (e também tentamos encontrar contraexemplos) mas sem sucesso.

Voltando ao último teorema, o corolário a seguir é uma consequência trivial:

Corolário 2.21. Se $T$ é um operador hipercíclico invertivel, então existem $M \subsetneq X$ subespaço e um operador linear invertível $A: X \rightarrow X$ tais que $T$ é $M$-hipercíclico e $T^{-1}$ é $A(M)$-hipercíclico.

Assim como o Teorema 2.16 nos mostrou uma direção para tentar resolver o Problema 3, o corolário anterior nos mostra uma direção para o Problema 1. será que a resposta para tal problema consiste em um operador invertível $A \in \mathcal{B}(X)$ tal que $A\left(M_{1}\right)=M_{2}$ ?

Com isso em mente, motivados pelo último teorema (e especialmente seu corolário) e pensando em solucionar (totalmente ou parcialmente) os Problemas 1, 2 e 3, fizemos a seguinte conjectura:

Conjectura. Sejam X um espaço de Banach e $M, N \subsetneq X$ subespaços fechados de dimensão infinita. Seja $T \in \mathcal{B}(X)$ um operador sub-hipercíclico para $M$ e $N$. Então existe $A: X \rightarrow X$ linear, invertivel tal que $A(M)=N$.

Tal conjectura é claramente inspirada no Lema 1.3, uma vez lembrado que se $T$ é um operador hipercíclico com vetor hipercíclico $x$, então orb $(x, T)$ é denso e linearmente independente (Lema 1.34 ; logo, o Lema 1.3 nos diz que duas órbitas quaisquer (inclusive de operadores diferentes) são isomorfas. Dessa maneira, pode-se dizer que a conjectura é uma adaptação pensada para o caso de operadores sub-hipercíclicos 10

\footnotetext{
${ }^{10}$ Observe que se tentássemos adaptar fielmente o Lema 1.3 para o caso de conjuntos densos em subespaços, obteríamos o seguinte: "Sejam $X$ um espaço de Banach, M, N $\subsetneq X$ subespaços fechados de dimensão infinita $e$ $X_{0} \subseteq M$ e $Y_{0} \subseteq N$ conjuntos enumeráveis, densos e linearmente independentes em $M$ e $N$, respectivamente. Tome $\varepsilon>0$. Então existe um operador linear invertivel $A$ em $X$ tal que $A\left(X_{0}\right)=Y_{0} e\|I-A\|<\varepsilon$." Sendo $A$ invertível, então temos que $A(M)=A\left(\overline{X_{0}}\right)=\overline{A\left(X_{0}\right)}=\overline{Y_{0}}=N$. Desta forma, o enunciado posto na conjectura é, de fato, um resultado análogo ao Lema 1.3 (lembrando também do Lema 2.9 .
} 
No entanto, veremos no exemplo a seguir que tal conjectura é falsa.

Exemplo 2.22. Sejam $X, Y$ espaços de Banach separáveis de dimensão infinita que não sejam isomorfos. Defina $Z:=X \oplus Y, M:=X \oplus\{0\}$ e $N:=\{0\} \oplus Y$. É claro que $Z$ é um espaço de Banach separável e $M, N \subsetneq Z$ são subespaços fechados de dimensão infinita. Observe ainda, a título de curiosidade, que $M \cap N=\{0\}$.

Pelo Teorema 2.17, existe $T \in \mathcal{B}(Z)$ invertível tal que $T$ é $M$-hipercíclico. Agora, pelo Teorema 2.16, existe $R \in \mathcal{B}(Z)$ invertível tal que $T$ é $R(N)$-hipercíclico. Se a conjectura anterior fosse verdadeira, então existiria $A \in \mathcal{B}(Z)$ invertível tal que $A(R(N))=M$. Ou seja, temos $(A \circ R)(N)=$ $M$. Logo, sendo $A \circ R$ uma composição de operadores invertíveis, temos que $M$ é isomorfo a $N$. Pela Proposição 1.8.3 de [22], podemos concluir que $X$ e $Y$ são isomorfos, o que é um absurdo tendo em vista a suposição inicial feita sobre estes espaços.

Apesar de nossa conjectura ser falsa, um outro resultado interessante e que generaliza o Lema 1.3 vale, como veremos a seguir:

Teorema 2.23 (Chalendar e Partington, [9]). Seja Y um subespaço fechado de um espaço de Banach $X$ separável de dimensão infinita. Sejam $V_{1}, W_{1} \subseteq Y$ subconjuntos densos, enumeráveis e linearmente independentes. Sejam agora $V_{2}, W_{2} \subseteq X$ subconjuntos enumeráveis que são linearmente independentes sobre $Y$ (isto é, os subconjuntos $p\left(V_{2}\right)$ e $p\left(W_{2}\right)$ são linearmente independentes em $X / Y$, onde $p: X \rightarrow X / Y$ é a projeção canônica) e tais que $V_{1} \cup V_{2}$ e $W_{1} \cup W_{2}$ são subconjuntos densos (e linearmente independentes) em $X$. Então existe um operador invertivel $L$ tal que $L\left(V_{1}\right)=$ $W_{1}$ e $L\left(V_{2}\right)=W_{2}$. Também existe um operador compacto $K$ que é injetivo, com imagem densa $e$ que também é tal que $K\left(V_{1}\right)=V_{2}$ e $K\left(W_{1}\right)=W_{2}$.

Tome $T \in \mathcal{B}(X)$ um operador hipercíclico. Suponha que $x, y \in H(T)$ são tais que $x$ e $y$ são também vetores $M$-hipercíclicos para $T$. Então pelo teorema acima temos que existe um operador invertível $L \in \mathcal{B}(X)$ tal que $L(\operatorname{orb}(x, T) \cap M)=\operatorname{orb}(y, T) \cap M$ e $L\left(V_{2}\right)=W_{2}$, onde $V_{2}=[\operatorname{orb}(x, T) \backslash(\operatorname{orb}(x, T) \cap M)]$ e $W_{2}=[\operatorname{orb}(y, T) \backslash \operatorname{orb}(y, T) \cap M]$.

Sob estas condições, temos que o teorema anterior é claramente um upgrade em relação ao Lema 1.3. De fato, com o Lema 1.3 podemos concluir apenas que existe $A \in \mathcal{B}(X)$ invertível tal que $A(\operatorname{orb}(x, T))=\operatorname{orb}(y, T)$; não necessariamente vale que $A(\operatorname{orb}(x, T) \cap M)=\operatorname{orb}(y, T) \cap M$.

Apesar desta melhoria, o Teorema 2.23 (e, por sua vez, o Lema 1.3 não funciona "tão bem" no caso de operadores estritamente sub-hipercíclicos (isto é, o operador não é hipercíclico), uma vez que por mais que orb $(x, T) \cap M$ seja densa em $M$, não teríamos orb $(x, T)$ densa em $X$, não satisfazendo assim uma das hipóteses do teorema. Ficaríamos, neste caso, na dependência da existência de um subconjunto $V \subseteq X \backslash M$ denso e tal que $p(V)$ é linearmente independente em $X / Y$. Assim, pelo teorema anterior, existiria um operador invertível $L \in \mathcal{B}(X)$ tal que $L(\operatorname{orb}(x, T) \cap M)=$ $\operatorname{orb}(y, T) \cap M($ e $L(V)=V)$.

Logo, por mais que o Teorema 2.23 mostre (sob certas condições) que duas órbitas dentro de um mesmo subespaço sejam isomorfas, ele também não nos ajuda a resolver os Problemas 1, 2 e 3 já vistos, uma vez que o teorema trata de só um mesmo subespaço e não de conjuntos em 
diferentes subespaços (como é o caso dos problemas mencionados). Como já vimos, o resultado ideal que, em teoria, generaliza o Teorema 2.23 para dois subespaços é falso (a saber, a conjectura feita anteriormente). No entanto, é interessante pensar se podemos melhorar o Teorema 2.23 de maneira a incluir um outro subespaço sob hipóteses mais restritas (e obter, assim, um resultado que seja um "meio termo" entre o Teorema 2.23 e a conjectura). Para exemplificar o que queremos dizer, vamos lembrar do Exemplo 2.22. Nele, o espaço de Banach em questão é claramente decomponível. Se adicionarmos a hipótese de $X$ ser indecomponível à conjectura, será que ela ainda é falsa? Além disso, será que podemos estender o Teorema 2.23 para espaços não-separáveis (ainda que sob diferentes hipóteses)? Neste caso, é interessante pensar em estender o teorema nesta direção pois, como vimos no Exemplo 2.3. temos que existem sim operadores sub-hipercíclicos em espaços não-separáveis (e veremos mais adiante um resultado melhor: todo espaço não-separável admite um operador sub-hipercíclico). Tais perguntas serão abordadas em pesquisas futuras.

Mudando agora completamente de assunto, no primeiro capítulo vimos, na Proposição 1.26 , como hiperciclicidade se "comporta bem" sob as quasi-conjugações. É natural, então, perguntar se acontece o mesmo com sub-hiperciclicidade. Vale ressaltar que, como todo operador hipercíclico é sub-hipercíclico (Teorema 2.6), então não é necessário analisar o que acontece com subhiperciclicidade quando $T$ é hipercíclico e quasi-conjugado a um $S$; precisamos considerar apenas que $T$ é sub-hipercíclico (e quasi-conjugado a $S$ ): seria, nesse caso, $S$ sub-hipercíclico? Se sim, para qual subespaço? Este é o assunto da próxima seção.

\subsection{Relações entre Hiperciclicidade e Sub-Hiperciclicidade via Quasi- Conjugações}

Levando em consideração o que foi dito no final da seção anterior e baseado na Proposição 1.26 , é natural fazermos a seguinte conjectura:

Conjectura. Sejam $X$ um espaço de Banach e $S, T \in \mathcal{B}(X)$. Suponha que $T$ seja quasi-conjugado a $S$ (via $\phi)$. Se $T$ é $M$-hipercíclico, então $S$ é $\phi(M)$-hipercíclico. Ainda, se $x \in X$ é um vetor $M$-hipercíclico para $T$, então $\phi(x)$ é um vetor $\phi(M)$-hipercíclico para $S$.

Vamos tentar elaborar uma demonstração para a conjectura acima:

DemonstraÇÃo: Seja $x$ um vetor $M$-hipercíclico para $T$. Mostremos que $\phi(x)$ é um vetor $\phi(M)$ hipercíclico para $S$. Tome $W$ um aberto não-vazio de $\phi(M)$. Então podemos escrever $W=$ $U \cap \phi(M)$, com $U$ um aberto de $X$. Como $\phi$ é contínuo e tem imagem densa, então $\phi^{-1}(U)$ é aberto e não-vazio.

Temos agora que $V=\phi^{-1}(U) \cap M$ é não-vazio (e, portanto, um aberto de $M$ ). De fato, se $V=\emptyset$, então $\emptyset=\phi(V)=\phi\left(\phi^{-1}(U) \cap M\right)=U \cap \phi(M)=W$, um absurdo.

Sendo $V$ não-vazio e um aberto de $M$, então existe $n \in \mathbb{N}$ tal que $T^{n} x \in V$. Logo, $\phi\left(T^{n} x\right) \in$ $\phi(V) \Rightarrow S^{n}(\phi x) \in W$, uma vez que $S \circ \phi=\phi \circ T$ e $\phi(V)=W$. Como $W$ era um aberto qualquer de $\phi(M)$, isto mostra que $S$ é $\phi(M)$-hipercíclico e que $\phi(x)$ é um vetor $\phi(M)$-hipercíclico para $S$.

Observe que a demonstração acima está tecnicamente correta. No entanto, um pequeno detalhe pode atrapalhar a conclusão desejada: considerando as hipóteses feitas na conjectura, o subespaço 
$\phi(M)$ produzido pode muito bem ser trivial $(\phi(M)=\{0\})$, ter dimensão finita, não ser fechado, ou ainda termos $\phi(M)=X$ (o que mostraria que $S$ é hipercíclico). Todos os casos não são de nosso interesse, como deixamos claro após a Definição 2.1 e na Proposição 2.8. Para ilustrar tal situação, vejamos o exemplo a seguir:

Exemplo 2.24. Seja $T$ um operador $M$-hipercíclico em um espaço de Banach $X$. Considerando o espaço $X \oplus X$, tome $p_{2}$ a projeção na segunda coordenada. Note que, de maneira análoga ao Exemplo 2.2, o operador $T \oplus I$ é $M \oplus\{0\}$-hipercíclico em $X \oplus X$. Agora, temos que $p_{2} \circ(T \oplus I)=I \circ p_{2}$ e, como $p_{2}$ é contínuo e possui imagem densa, então $T \oplus I$ é quasi-conjugado a $I$ via $p_{2}$. No entanto, $\{0\}$ é um espaço de dimensão finita e, como mostra a Proposição 2.8, I não é $\{0\}$-hipercíclico.

Visto tal exemplo, cabe a pergunta: que mudanças precisamos fazer nas hipóteses da conjectura de maneira a eliminar os casos já citados em que $\phi(M)$ não é o desejado? Com o exemplo acima, fica claro que supor apenas que $\phi$ é contínua e com imagem densa não basta. Dessa forma, precisamos colocar hipóteses adicionais sobre $\phi$ de maneira a impossibilitar que $\phi(M)$ seja trivial, tenha dimensão finita ou não seja fechado. Como veremos na proposição abaixo, se supusermos que $\phi$ (substituído por $i$ na proposição) seja linear, injetor e com imagem fechada, então tais problemas estão resolvidos:

Proposição 2.25. Sejam $X, Y$ espaços de Banach de dimensão infinita e $T \in \mathcal{B}(X), S \in \mathcal{B}(Y)$. Seja $i: X \rightarrow Y$ um operador linear limitado tal que $i$ é injetor, tem imagem fechada e satisfaz $S \circ i=i \circ T$. Então $T$ é $M$-hipercíclico se, e somente se, $S$ é $i(M)$-hipercíclico.

DemonstraÇÃo: Observe que sendo $i$ linear, contínuo, injetor e com imagem fechada, então pelo Teorema da Aplicação Aberta temos que $\left.i\right|_{M}: M \rightarrow i(M)$ é um operador invertível. Logo, se $j=\left(\left.i\right|_{M}\right)^{-1}$, então usando a hipótese obtemos que $j \circ S=T \circ j$. Por isso, para mostrar a proposição, basta mostrarmos apenas uma das implicações.

Suponha que $T$ seja $M$-hipercíclico e tome $x$ um vetor $M$-hipercíclico para $T$. Vamos mostrar que $i(x)$ é um vetor $i(M)$-hipercíclico para $S$. De fato, seja $i(y) \in i(M)$. Como $x$ é um vetor $M$-hipercíclico, existe $n \in \mathbb{N}$ tal que $T^{n} x \in M$ e $\left\|T^{n} x-y\right\|<\varepsilon /\|i\|$ (uma vez que $y \in M$ ). Portanto:

$$
\begin{aligned}
\left\|S^{n} i(x)-i(y)\right\| & =\left\|i\left(T^{n} x\right)-i(y)\right\|=\left\|i\left(T^{n} x-y\right)\right\| \\
& \leq\|i\|\left\|T^{n} x-y\right\| \\
& <\varepsilon
\end{aligned}
$$

uma vez que, como $S \circ i=i \circ T$, então $S^{n} \circ i=i \circ T^{n}$.

Observe que sendo $i$ injetor e com imagem fechada, então temos que $i(M)$ é um subespaço de dimensão infinita e fechado. Assim, a desigualdade em destaque acima nos mostra que $S$ é $i(M)$-hipercíclico, como desejado. 
Observe que trocamos, na proposição acima, a hipótese de $i$ ter imagem densa pela hipótese de $i$ ser linear. O motivo é simples: para garantirmos que $i(M)$ tenha dimensão infinita, colocamos a hipótese de que $i$ é injetor. Além disso, precisamos também que $i(M)$ seja fechado. Para isso, basta supor que $i$ é linear e tem imagem fechada (como vimos na proposição).

Agora, se tirarmos a linearidade e supormos que $i$ é injetor, com imagem fechada e densa, estamos supondo então que $\phi$ é uma bijeção contínua. Tal fato, junto com a hipótese de que $\phi \circ T=S \circ \phi$, torna toda a ideia de quasi-conjugações um tanto quanto trivial. Ao substituirmos a hipótese de $\phi$ ter imagem densa pela linearidade, conseguimos ter alguns resultados que não obteríamos se $\phi$ tivesse imagem densa, como veremos a seguir:

Corolário 2.26. Sejam $X$ um espaço de Banach não-reflexivo, $T \in \mathcal{B}(X)$ e denote por $i$ a imersão isométrica canônica $i: X \rightarrow X^{* *}$. Então $T$ é um operador $M$-hipercíclico se, e somente se, $T^{* *} e ́$ um operador $i(M)$-hipercíclico em $X^{* *}$.

Demonstração: Basta lembrar que se $z^{*} \in X^{*}$, então $T^{* *} i(x)\left(z^{*}\right)=i(x)\left(T^{*} z^{*}\right)=T^{*}\left(z^{*}\right)(x)=$ $z^{*}(T x)=i(T x)\left(z^{*}\right)$ e, portanto, $T^{* *} i(x)=i(T x)$, para todo $x \in X$, de onde segue que $T^{* *} \circ i=i \circ T$. Como a imersão canônica é obviamente limitada, injetora e com imagem fechada, o corolário segue imediatamente da proposição anterior.

Note que podemos também considerar $T$ hipercíclico na Proposição 2.25. É claro que, como dissemos no começo desta seção, temos que tomar o cuidado para não cairmos no caso que já apresentamos (a Proposição 1.26); isto é, supor que $T$ é hipercíclico e quasi-conjugado a um $S$. Portanto, para considerar $T$ hipercíclico, tomaremos cuidado com as hipóteses que usaremos (justamente para não repetir a Proposição 1.26), do mesmo jeito que fizemos com a Proposição 2.25 .

Proposição 2.27. Sejam $X, Y$ espaços de Banach de dimensão infinita e $T \in \mathcal{B}(X), S \in \mathcal{B}(Y)$. Seja $i: X \rightarrow Y$ um operador linear limitado tal que $i$ é injetor, tem imagem fechada, não é sobrejetor e satisfaz $S \circ i=i \circ T$. Então $T$ é hipercíclico se, e somente se, $S$ é $i(X)$-hipercíclico.

Demonstração: A demonstração é totalmente análoga à demonstração da Proposição $\left.2.25\right|^{11}$

Observe que a hipótese de que $i$ não é sobrejetor é colocada na proposição acima pois se $i(X)=$ $Y$, então $S$ seria $Y$-hipercíclico (ou seja, hipercíclico). Além disso, se $i(X)=Y$, então $i$ seria contínuo com imagem densa (uma vez que é sobrejetor); como $T$ é hipercíclico, a Proposição 1.26 já nos mostra que $S$ também é hipercíclico. Logo, não há necessidade de considerar esse caso.

No espírito do Corolário 2.26, temos mais um corolário envolvendo o bidual:

Corolário 2.28. Sejam $X$ um espaço de Banach não-reflexivo, $T \in \mathcal{B}(X)$ e denote por $i$ a imersão isométrica canônica $i: X \rightarrow X^{* *}$. Então $T$ é um operador hipercíclico se, e somente se, $T^{* *}$ é um operador $i(M)$-hipercíclico em $X^{* *}$.

\footnotetext{
${ }^{11}$ Como dito após a Definição 2.1 se tomarmos a liberdade de dizer que um operador hipercíclico é um operador $X$-hipercíclico, então esta proposição segue imediamente da Proposição 2.25
} 
DemonstraÇÃo: Note que, ao supormos $X$ não-reflexivo, temos que $i(X) \neq X$. Logo, assim como o Corolário 2.26, uma vez lembrado que $T^{* *} \circ i=i \circ T$, o resultado segue da proposição anterior.

Observe agora que o Exemplo 2.3 nada mais é que um caso particular do corolário acima: tomando $X=c_{0}$, temos que $X^{* *}=\ell_{\infty}$. Logo, como $B_{w} \in \mathcal{B}\left(c_{0}\right)$, não é difícil verificar que $\left(B_{w}\right)^{* *}=B_{w} \in \mathcal{B}\left(\ell_{\infty}\right)$ e que $i\left(c_{0}\right)=c_{0} \subseteq \ell_{\infty}$.

Corolário 2.29. Seja $X$ um espaço de Banach separável. Então existem um operador $S \in \mathcal{B}\left(\ell_{\infty}\right)$ e um subespaço $M \subseteq \ell_{\infty}$ tais que $S$ é $M$-hipercíclico e $X$ é isométrico a $M$.

DemonstraÇÃo: Pelo Teorema 1.6, existe uma imersão isométrica $i: X \rightarrow \ell_{\infty}$. Denote $M=$ $i(X)$. Seja $T$ um operador hipercíclico em $X$. Note que $S_{1}: M \rightarrow \ell_{\infty}$ definido por $S_{1}(i(x))=i(T x)$ é um operador bem definido, linear e limitado (pois $T$ é linear, limitado e $i$ é imersão isométrica).

Como $\ell_{\infty}$ é isometricamente injetivo (Proposição 1.11), então existe $S$ que estende $S_{1}$ e é tal que $\left.S\right|_{M}=S_{1}$. Logo, segue que $S \circ i=i \circ T$. Pela proposição anterior, como $i(X)=M$, segue que $S$ é $M$-hipercíclico, como desejado.

Sabemos que todo operador hipercíclico é sub-hipercíclico. Logo, é trivial achar exemplos de operadores sub-hipercíclicos em espaços separáveis (uma vez que todo espaço separável admite um operador hipercíclico, vide o Corolário 1.28). Mostramos, no Exemplo 2.3, um exemplo de um operador sub-hipercíclico em $\ell_{\infty}$. No entanto, tal exemplo ainda é um tanto trivial, uma vez que, além do fato de existir a imersão $i: c_{0} \rightarrow \ell_{\infty}$ como comentamos no parágrafo anterior, também devemos levar em conta que $\ell_{\infty}$ é injetivo (vide a Definição 1.10 e Proposição 1.11).

Construir exemplos de operadores sub-hipercíclicos em espaços injetivos também é fácil, uma vez que todo espaço de Banach possui uma sequência básica (vide o Teorema 1.5). Se $X$ é injetivo e $M \subseteq X$ é o subespaço fechado gerado por tal sequência básica, então $M$ admite um operador hipercíclico $T: M \rightarrow M \subseteq X$ e daí não é difícil ver que a extensão $\tilde{T} \in \mathcal{B}(X)$ (obtida pela injetividade de $X$ ) é $M$-hipercíclico.

Tome agora um operador hipercíclico qualquer em $\ell_{1}$ (por exemplo, $B_{w}$, para $|w|>1$, vide o Exemplo 1.18. Denote por $T$ tal operador. Pelo Corolário 2.28, $T^{* *}$ é um exemplo de um operador sub-hipercíclico em $\left(\ell_{\infty}\right)^{*}$. Apesar de não sabermos se $\left(\ell_{\infty}\right)^{*}$ é injetivo ou não, ele é tal que $l_{1}$ é um subespaço complementado em $\left(\ell_{\infty}\right)^{*}$, já que $\left(\ell_{\infty}\right)^{*}=\ell_{1} \oplus c_{0}^{\perp}$ (vide a Proposição 1.7). Apesar disso, vale destacar que este é o primeiro exemplo conhecido de um operador sub-hipercíclico em $\left(\ell_{\infty}\right)^{*}$.

Assim como no caso de espaços injetivos, é trivial ver que espaços que possuem um subespaço de dimensão infinita complementado (isto é, espaços decomponíveis) são espaços fáceis de se construir exemplos de operadores sub-hipercíclicos. Por isso, o exemplo citado no parágrafo anterior, assim como os outros, ainda é um tanto quanto trivial. Isto nos leva a pergunta: será que é possível acharmos exemplos de operadores sub-hipercíclicos em qualquer espaço de Banach (não-separável)? Ou será que operadores sub-hipercíclicos em espaços não-separáveis ficam restritos a estes casos "triviais" (o espaço em questão ser injetivo ou decomponível)? 
De maneira a responder tais perguntas mostraremos, na próxima seção, que todo espaço de Banach admite um operador sub-hipercíclico. Além disso, veremos também que se $M \subsetneq X$ é um subespaço separável de dimensão infinita, então existe $T \in \mathcal{B}(X)$ tal que $T$ é $M$-hipercíclico, generalizando o Teorema 2.17 para espaços de Banach não necessariamente separáveis.

\subsection{Existência de Operadores Sub-Hipercíclicos}

Vimos, no Teorema 1.27, que todo espaço de Banach separável admite um operador hipercíclico (e, portanto, um operador sub-hipercíclico). Fortemente inspirado pela demonstração deste teorema, conseguimos adaptá-la e mostrar que todo espaço de Banach não-separável admite um operador sub-hipercíclico. Tal adaptação foi possível, em grande parte, graças ao Teorema de Hahn-Banach, como veremos a seguir.

Teorema 2.30. Todo espaço de Banach não-separável de dimensão infinita admite um operador que é sub-hipercíclico.

DemonstraçÃo: Pelo Teorema 1.5, existe uma sequência básica normalizada em $X$. Denote por $M$ o subespaço fechado de dimensão infinita gerado por essa sequência básica. Note que, sendo $M$ um subespaço fechado gerado por uma sequência básica, então podemos olhar para $M$ como um espaço de Banach separável. Uma vez que $X$ não é separável, então é claro que $X \neq M$.

Pelo Teorema 1.8, existem sequências $\left\{x_{n}\right\}_{n=1}^{\infty} \subseteq M$ e $\left\{x_{n}^{*}\right\}_{n=1}^{\infty} \subseteq M^{*}$ tais que:

(a) $x_{i}^{*}\left(x_{j}\right)=\delta_{i j}$, onde $\delta_{i j}$ é o delta de Kronecker.

(b) $\operatorname{span}\left\{x_{n}: n \in \mathbb{N}\right\}$ é denso em $M$.

(c) se $x \in M$ é tal que $x_{n}^{*}(x)=0$ para todo $n \in \mathbb{N}$, então $x=0$.

(d) $\left\|x_{n}\right\|=1$, para todo $n \in \mathbb{N}$ e $\sup _{n \in \mathbb{N}}\left\|x_{n}^{*}\right\|=C<\infty$.

Pelo Teorema de Hahn-Banach, podemos estender cada $x_{n}^{*}$ à $X$, preservando sua norma. Para simplificar, tais extensões também serão denotadas por $x_{n}^{*} \in X^{*}$. Observe que $\left.x_{n}^{*}\right|_{M}$ continua satisfazendo as propriedades listadas acima.

Seja agora $T: X \rightarrow X$ dado por

$$
T x=x+\sum_{n=1}^{\infty} 2^{-n} x_{n+1}^{*}(x) x_{n}
$$

Temos que $T$ é linear e, de maneira similar ao Teorema 1.27, temos que as propriedades listadas oriundas do Teorema 1.8 implicam que $T$ é um operador limitado em $X$. De fato, pelo Teorema de Hahn-Banach temos que $\left\|x_{n+1}^{*}\right\|=\left\|\left.x_{n+1}^{*}\right|_{M}\right\| \leq C<\infty$. Portanto, tomando $x \in B_{X}$, temos:

$$
\begin{aligned}
\left\|\sum_{n=1}^{\infty} 2^{-n} x_{n+1}^{*}(x) x_{n}\right\| & \leq \sum_{n=1}^{\infty}\left\|2^{-n} x_{n+1}^{*}(x) x_{n}\right\|=\sum_{n=1}^{\infty} 2^{-n}\left|x_{n+1}^{*}(x)\right|\left\|x_{n}\right\| \\
& \leq \sum_{n=1}^{\infty} 2^{-n}\left\|x_{n+1}^{*}\right\|\|x\| \leq \sum_{n=1}^{\infty} 2^{-n} C<\infty
\end{aligned}
$$


uma vez que, pelo item (d), $\left\|x_{n}\right\|=1$, para todo $n \in \mathbb{N}$. Logo, segue que $T$ é limitado.

Observe agora que $T$ é $M$-invariante. De fato, seja $m \in M$. Como $\operatorname{span}\left\{x_{n}: n \in \mathbb{N}\right\}$ é denso em $M$, então existe uma sequência $\left(y_{n}\right)_{n \in \mathbb{N}} \subseteq \operatorname{span}\left\{x_{n}: n \in \mathbb{N}\right\}$ tal que $y_{n} \rightarrow m$. Note que se mostrarmos que $T\left(y_{n}\right) \in M$, para todo $n \in \mathbb{N}$, seguirá que $T(m) \in M$ : de fato, como $T$ é contínuo, então $T\left(y_{n}\right) \rightarrow T(m)$. Agora, se $T\left(y_{n}\right) \in M$, para todo $n \in \mathbb{N}$, então $T(m) \in \bar{M}$. Sendo $M$ fechado, segue que $T(m) \in M$, mostrando que $T$ é $M$-invariante. Provemos, então, que $T\left(y_{n}\right) \in M$, para todo $n \in \mathbb{N}$.

Fixe um $n \in \mathbb{N}$ e tome $y_{n} \in \operatorname{span}\left\{x_{n}: n \in \mathbb{N}\right\} \subseteq M$. Como $T y_{n}=y_{n}+\sum_{n=1}^{\infty} 2^{-n} x_{n+1}^{*}\left(y_{n}\right) x_{n}$, então é claro que basta mostrarmos que $\sum_{n=1}^{\infty} 2^{-n} x_{n+1}^{*}\left(y_{n}\right) x_{n} \in M$, uma vez que $M$ é um subespaço. Para isso, escreva $y_{n}=\sum_{j=1}^{k} \lambda_{j} x_{n_{j}}$. Temos:

$$
\sum_{n=1}^{\infty} 2^{-n} x_{n+1}^{*}\left(y_{n}\right) x_{n}=\sum_{n=1}^{\infty} 2^{-n} x_{n+1}^{*}\left(\sum_{j=1}^{k} \lambda_{j} x_{n_{j}}\right) x_{n}=\sum_{n=1}^{\infty} \sum_{j=1}^{k} 2^{-n} \lambda_{j} x_{n+1}^{*}\left(x_{n_{j}}\right) x_{n}
$$

Como dito anteriormente, $\left.x_{n}^{*}\right|_{M}$ continua satisfazendo as propriedades do Teorema 1.8. Logo, como $x_{j} \in M$, pelo item (a) de tal teorema temos que $x_{i}^{*}\left(x_{j}\right)=\delta_{i j}$. Assim obtemos que:

$$
\sum_{n=1}^{\infty} \sum_{j=1}^{k} 2^{-n} \lambda_{j} x_{n+1}^{*}\left(x_{n_{j}}\right) x_{n}=\sum_{j=1}^{k} 2^{-\left(n_{j}-1\right)} \lambda_{j} x_{n_{j}-1}
$$

Não é difícil ver que $\sum_{j=1}^{k} 2^{-\left(n_{j}-1\right)} \lambda_{j} x_{n_{j}-1} \in \operatorname{span}\left\{x_{n}: n \in \mathbb{N}\right\} \subseteq M$. Isto mostra que $\sum_{n=1}^{\infty} 2^{-n} x_{n+1}^{*}\left(y_{n}\right) x_{n} \in M$ e, portanto, que $T$ é $M$-invariante, como desejado.

Logo, o operador $\left.T\right|_{M}: M \rightarrow M$ está bem definido. Vamos proceder agora de maneira parecida ao Teorema 1.27. Seja $S: \ell_{1} \rightarrow \ell_{1}$ dado por

$$
S\left(\left(\alpha_{n}\right)_{n \in \mathbb{N}}\right)=\left(\alpha_{1}+\frac{1}{2} \alpha_{2}, \alpha_{2}+\frac{1}{2^{2}} \alpha_{3}, \alpha_{3}+\frac{1}{2^{3}} \alpha_{4}, \ldots\right)
$$

Note, novamente, que $S$ é da forma $I+B_{w_{n}}, \operatorname{com} w_{n}=1 / 2^{n-1}$. Como $\sup \left|w_{n}\right|<\infty$, então pelo Exemplo 1.22 temos que $S$ é mixing e, portanto, hipercíclico. Tome agora $\phi: \ell_{1} \rightarrow M$ dado por

$$
\phi\left(\left(\alpha_{n}\right)_{n \in \mathbb{N}}\right)=\sum_{n=1}^{\infty} \alpha_{n} x_{n}
$$

Não é difícil ver que $\phi$ é contínua e, tendo em mente o item (b) do Teorema 1.8 , temos que $\phi$ possui imagem densa em $M \stackrel{12}{12}$ Agora, temos que

\footnotetext{
${ }^{12}$ Temos que $\phi$ é contínua pois $\left\|\sum_{n=1}^{\infty} \alpha_{n} x_{n}\right\| \leq \sum_{n=1}^{\infty}\left\|\alpha_{n} x_{n}\right\|=\sum_{n=1}^{\infty}\left|\alpha_{n}\right|\left\|x_{n}\right\|=\sum_{n=1}^{\infty}\left|\alpha_{n}\right|<\infty$, uma vez que $\left\|x_{n}\right\|=1$ e $\left(\alpha_{n}\right)_{n \in \mathbb{N}} \in \ell_{1}$.
} 


$$
\begin{aligned}
\left.T\right|_{M} \circ \phi\left(\left(\alpha_{n}\right)_{n \in \mathbb{N}}\right) & =\left.T\right|_{M}\left(\sum_{n=1}^{\infty} \alpha_{n} x_{n}\right)=\left.\sum_{n=1}^{\infty} \alpha_{n} T\right|_{M}\left(x_{n}\right)=\alpha_{1} x_{1}+\sum_{n=2}^{\infty} \alpha_{n}\left(x_{n}+2^{-(n-1)} x_{n-1}\right) \\
& =\alpha_{1} x_{1}+\sum_{n=2}^{\infty}\left(\alpha_{n} x_{n}+\alpha_{n} 2^{-(n-1)} x_{n}\right)=\alpha_{1} x_{1}+\sum_{n=2}^{\infty} \alpha_{n} x_{n}+\sum_{n=2}^{\infty} \alpha_{n} 2^{-(n-1)} x_{n-1} \\
& =\sum_{n=1}^{\infty} \alpha_{n} x_{n}+\sum_{n=1}^{\infty} \alpha_{n+1} 2^{-n} x_{n}=\phi\left(\left(\alpha_{n}\right)_{n \in \mathbb{N}}\right)+\phi\left(\left(2^{-n} \alpha_{n+1}\right)_{n \in \mathbb{N}}\right) \\
& =\phi\left(\left(\alpha_{n}+2^{-n} \alpha_{n+1}\right)_{n \in \mathbb{N}}\right)=\phi \circ S\left(\left(\alpha_{n}\right)_{n \in \mathbb{N}}\right)
\end{aligned}
$$

Logo, $\left.T\right|_{M} \circ \phi=\phi \circ S$. Portanto, $S$ é quasi-conjugado a $\left.T\right|_{M}$. Sendo $S$ hipercíclico, então pela Proposição 1.26 temos que $\left.T\right|_{M}$ é hipercíclico. Daí, lembrando da Observação 2.4 , segue que $T$ é $M$-hipercíclico, terminando a demonstração.

Com este resultado, podemos afirmar que todo espaço de Banach admite um operador subhipercíclico:

Teorema 2.31. Todo espaço de Banach admite um operador sub-hipercíclico.

Demonstração: Se o espaço for separável, então o espaço admite um operador hipercíclico (vide o Teorema 1.27). Como todo operador hipercíclico é sub-hipercíclico (vide o Teorema 2.7), segue o desejado.

Agora, se o espaço em questão não for separável, o resultado segue do teorema anterior.

Vimos na demonstração do último teorema que o $T$ e o $M$ construídos são tais que $T$ é $M$ invariante. Como dito no começo do capítulo, isto torna o operador construído um tanto quanto "simples" demais: uma vez mostrado que $T$ era $M$-invariante, mostrar que $T$ é $M$-hipercíclico virou um exercício de mostrar que $\left.T\right|_{M}$ é hipercíclico (em $M$ ). Um tópico interessante para futuras pesquisas, portanto, é tentar garantir a existência, em um espaço de Banach qualquer, de um operador $M$-hipercíclico que não necessariamente seja $M$-invariante.

Por outro lado, como o subespaço $M$ foi obtido através de um teorema de existência (a saber, o Teorema 1.5), então pouco sabemos a seu respeito. Ele pode ser, por exemplo, complementado em $X$ - o que tornaria o operador $T$ construído um tanto quanto trivial, como discutido no final da seção anterior 13 No entanto, se tomarmos $X$ como sendo um espaço indecomponível, então eliminamos tal possibilidade. O mesmo vale para a injetividade também discutida anteriormente.

Ao fim de toda essa discussão ao redor do subespaço $M$ obtido através do Teorema 1.5, se nos atentarmos à demonstração do Teorema 2.30, veremos que usamos o Teorema 1.5 apenas para garantir a existência de um subespaço fechado de dimensão infinita separável em qualquer espaço de Banach $X$. Como já dito, uma vez que tal teorema garante apenas a existência de tal subespaço

\footnotetext{
${ }^{13}$ Tomando $X=\left(\ell_{\infty}\right)^{*}$, por exemplo, o subespaço $M$ obtido através do Teorema 1.5 pode muito bem ser $M=\ell_{1}$, que é tal que $\left(\ell_{\infty}\right)^{*}=\ell_{1} \oplus c_{0}^{\perp}$, como já discutimos.
} 
e não nos fornece nenhuma informação adicional, não utilizamos nenhuma propriedade intrínseca de tal $M$ obtido - com exceção da separabilidade. Portanto, podemos muito bem substituir tal $M$ por outro subespaço qualquer, desde que tal subespaço seja, obviamente, separável e de dimensão infinita 14 Assim, se estivermos de posse de tal subespaço desde o começo, sem a necessidade de garantir a sua existência via o Teorema 1.5. estaríamos efetivamente "pulando" o primeiro parágrafo da demonstração do Teorema 2.30 .

Com esse argumento, fica provado o seguinte teorema:

Teorema 2.32. Sejam $X$ um espaço de Banach de dimensão infinita e $M \neq X$ um subespaço fechado de dimensão infinita e separável. Então existe $T \in \mathcal{B}(X)$ tal que $T$ é sub-hipercíclico para $M$.

Observe que o teorema acima é uma generalização do Teorema 2.17, ainda que, no caso em que $X$ seja separável, o teorema acima não nos permita concluir que $T$ é invertível e que satisfaz o HC (ao contrário do Teorema 2.17) 15

Com este resultado, podemos concluir que sub-hiperciclicidade se comporta do mesmo jeito que hiperciclicidade no seguinte sentido: sempre que há condições "mínimas", então o espaço (ou subespaço) admite um operador hipercíclico (ou sub-hipercíclico). Isto é, no caso de hiperciclicidade, sempre que o espaço em questão é separável e de dimensão infinita (duas condições necessárias para hiperciclicidade), então tal espaço admite um operador hipercíclico. De maneira semelhante, quando o subespaço em questão é separável e de dimensão infinita (analogamente, condições necessárias para sub-hiperciclicidade), então o subespaço em questão admite um operador sub-hipercíclico ${ }^{16}$

Voltaremos a falar destes dois resultados no próximo capítulo, após introduzirmos o Critério de Sub-Hiperciclicidade. Veremos que os operadores obtidos pelos dois teoremas anteriores satisfazem tal critério, deixando assim o Teorema 2.30 muito mais "próximo" de sua versão para operadores hipercíclicos, a saber, o Teorema 1.27 .

\subsection{Densidade de Operadores Sub-Hipercíclicos em $\mathcal{B}(X)$}

Seja $X$ um espaço de Banach de dimensão infinita. Acabamos de ver no Teorema 2.32 que, para todo subespaço $M \subsetneq X$ fechado, separável e de dimensão infinita, existe um operador $T$ que é $M$-hipercíclico. Levando em conta que todo espaço de Banach (de dimensão infinita) contém uma vasta quantidade de subespaços fechados (de dimensão infinita), podemos então esperar que também tenhamos uma "grande oferta" de operadores sub-hipercíclicos. Agora, o que seria matematicamente tal "grande oferta"?

A interpretação mais natural seria considerar a sua densidade: isto é, seria o conjunto de operadores sub-hipercíclicos em $X$ denso em $\mathcal{B}(X)$ ? Vimos no Lema 2.11 que todo operador subhipercíclico é tal que $\|T\|>1$. Logo, se considerarmos $\mathcal{B}(X)$ munido da topologia usual de operador,

\footnotetext{
${ }^{14}$ Condições sobre $M$ que, como já vimos neste capítulo, são necessárias para que exista um operador $T$ que seja $M$-hipercíclico.

${ }^{15}$ Observe que a única conclusão realmente "perdida" é a de que $T$ é invertível, uma vez que não há como concluir $T \in \mathcal{B}(X)$ hipercíclico - muito menos satisfazendo o $\mathrm{HC}$ - já que podemos muito bem supor $X$ como sendo um espaço de Banach não-separável.

${ }^{16}$ Apenas para deixar claro, quando dizemos, neste parágrafo, que um subespaço $M \subsetneq X$ "admite um operador sub-hipercíclico", queremos dizer que existe um operador $T \in \mathcal{B}(X)$ tal que $T$ é $M$-hipercíclico.
} 
então é claro que o conjunto de operadores sub-hipercíclicos não pode ser denso em $\mathcal{B}(X)$.

No entanto, podemos colocar em $\mathcal{B}(X)$ uma outra topologia bem conhecida - a saber, a topologia da convergência pontual de operadores, também conhecida como SOT ${ }^{17}$ Considerando $\mathcal{B}(X)$ munido desta topologia, veremos nesta seção que o conjunto dos operadores sub-hipercíclicos é sim denso em $\mathcal{B}(X)$, indo de encontro com a intuição de "grande oferta" dita anteriormente. Vale ressaltar que tal fenômeno já acontece com operadores hipercíclicos em espaços separáveis (isto é, quando $\mathcal{B}(X)$ está munido desta topologia, o conjunto dos operadores hipercíclicos é denso) ${ }^{18}$ Por isso é natural que tal resultado seja estendido para operadores sub-hipercíclicos.

De maneira a esclarecer as ideias, vamos agora definir a topologia SOT. Sejam $X$ um espaço de Banach qualquer e $\mathcal{B}(X)$ o conjunto dos operadores lineares contínuos em $X$. Dado $T \in \mathcal{B}(X)$ e um $n \geq 1$ qualquer, um sistema de vizinhanças para $T$ nesta topologia é definido por

$$
U_{x_{1}, \ldots, x_{n}}(T, \varepsilon)=\left\{S \in \mathcal{B}(X):\left\|T x_{k}-S x_{k}\right\|_{X}<\varepsilon, \text { para } k=1, \ldots, n\right\}
$$

onde $\left\{x_{1}, \ldots, x_{n}\right\} \subseteq X$ é um conjunto linearmente independente qualquer e $\|\cdot\|_{X}$ denota, evidentemente, a norma de $X$. Dessa forma, uma sequência $\left(T_{n}\right)_{n \in \mathbb{N}} \subseteq \mathcal{B}(X)$ converge nesta topologia para um operador $T \in \mathcal{B}(X)$ se, e somente se, $T_{n} x \stackrel{\|\cdot\|}{\longrightarrow} T x$, para todo $x \in X$ (e é por este motivo que tal topologia é conhecida por topologia da convergência pontual, como dito anteriormente). Uma vez definida tal topologia, quando dissermos que um conjunto é SOT-denso queremos dizer que o conjunto em questão é denso em $\mathcal{B}(X)$ quando munido da topologia SOT.

Para mostrarmos que o conjunto dos operadores sub-hipercíclicos é SOT-denso em $\mathcal{B}(X)$, precisaremos definir um conjunto que nos auxiliará nesta tarefa. Dados $X$ um espaço de Banach e $T \in \mathcal{B}(X)$, o conjunto $\mathcal{S}(T)$ é definido por

$$
\mathcal{S}(T)=\left\{A^{-1} \circ T \circ A ; A: X \rightarrow X \text { linear invertível }\right\}
$$

A proposição a seguir nos fornece condições suficientes para que $\mathcal{S}(T)$ seja SOT-denso em $\mathcal{B}(X)$.

Proposição 2.33 (Proposição 8.16 de [14]). Sejam X um espaço de Banach de dimensão infinita e $T \in \mathcal{B}(X)$. Suponha que, para todo $n \geq 1$, existam vetores $x_{1}, \ldots, x_{n} \in X$ tais que

$$
\left\{x_{1}, \ldots, x_{n}, T x_{1}, \ldots T x_{n}\right\}
$$

é um conjunto linearmente independente. Então $\mathcal{S}(T)$ é SOT-denso em $\mathcal{B}(X)$.

Com base na proposição acima, podemos demonstrar o resultado principal desta seção:

Teorema 2.34. Seja X um espaço de Banach de dimensão infinita. Então o conjunto dos operadores sub-hipercíclicos é SOT-denso em $\mathcal{B}(X)$.

Demonstração: Se $X$ for separável, então o conjunto dos operadores hipercíclicos é SOT-denso em $\mathcal{B}(X)$ (ver [14, Teorema 8.18, pp. 224-225]). Como todo operador hipercíclico é sub-hipercíclico (vide o Teorema 2.7) então neste caso obtemos o desejado.

\footnotetext{
${ }^{17}$ Uma abreviação do nome em inglês: strong operator topology.

${ }^{18}$ Para uma referência neste resultado, ver [14, Teorema 8.18, pp. 224-225].
} 
Suponha então que $X$ não seja separável. Pelo Teorema 2.30 existe um operador $T$ que é subhipercíclico em $X$. Seja $A: X \rightarrow X$ um operador linear invertível. Como $A^{-1} \circ T=\left(A^{-1} \circ T \circ\right.$ $A) \circ A^{-1}$ e $A^{-1}$ é invertível (e, portanto, injetor com imagem fechada), então pela Proposição 2.25 temos que $A^{-1} \circ T \circ A$ é um operador sub-hipercíclico em $X$. Logo, todo operador em $\mathcal{S}(T)$ é subhipercíclico em $X$. Assim, o desejado seguirá imediatamente se mostrarmos que $\mathcal{S}(T)$ é SOT-denso em $\mathcal{B}(X)$.

Se $x$ é um vetor sub-hipercíclico para $T$, então pelo Lema 2.9 temos que orb $(x, T)$ é linearmente independente. Logo, dado $n \geq 1$, temos que o conjunto $\left\{x, T x, \ldots, T^{2 n-1} x\right\}$ é linearmente independente. Rearranjando convenientemente os termos deste conjunto, obtemos que $\left\{x, T^{2} x, T^{4} x, \ldots, T^{2 n-2} x, T x, T^{3} x, T^{5} x, \ldots, T^{2 n-1} x\right\}$ é um conjunto linearmente independente. Denotando $x_{k}=T^{2 k-2} x$, pela proposição anterior temos então que $\mathcal{S}(T)$ é SOT-denso em $\mathcal{B}(X)$, mostrando o desejado.

É importante dizer que a demonstração do teorema acima é totalmente baseada na demonstração feita para operadores hipercíclicos (a saber, a demonstração encontrada em [14, Teorema 8.18, pp. 224-225] citada anteriormente). Apesar disso, vale frisar que pudemos apenas nos basear em tal demonstração pois obtivemos, ao longo deste texto, resultados para operadores sub-hipercíclicos análogos aos de operadores hipercíclicos, vide o Teorema 2.30 (que é o equivalente ao Teorema 1.27 para operadores hipercíclicos) e a Proposição 2.25 (que é o equivalente à Proposição 1.26 para operadores hipercíclicos). Sem tais resultados, não conseguiríamos nos basear e seguir a demonstração do caso hipercíclico de maneira tão fiel quanto seguimos. 


\section{Capítulo 3}

\section{O Critério de Sub-Hiperciclicidade}

Vimos, no capítulo anterior, alguns resultados de operadores hipercíclicos que foram "estendidos" (guardadas as devidas proporções) para operadores sub-hipercíclicos. Entre eles, podemos citar o Lema 2.9 , o Teorema 2.30 e os resultados envolvendo quasi-conjugações.

No primeiro capítulo vimos como o Critério de Hiperciclicidade é uma ferramenta importantíssima na teoria de operadores hipercíclicos - e como a questão de que se o Critério era também uma condição necessária foi o principal problema nesta área durante muitos anos. Tendo em mente os resultados "estendidos" citados no parágrafo anterior, é natural pensarmos em estender o Critério para operadores sub-hipercíclicos.

Pensando nisso, logo após introduzir a noção de sub-hiperciclicidade em [18], os autores deste artigo enunciaram e demonstraram um Critério de Sub-Hiperciclicidade. Tal critério, como veremos na primeira seção deste capítulo, é claramente baseado no HC. Veremos também as diferenças entre ele e o HC, assim como responderemos a pergunta natural: todo operador sub-hipercíclico satisfaz o tal Critério de Sub-Hiperciclicidade?

\subsection{Sub-Transitividade e o Critério de Sub-Hiperciclicidade}

Vimos no Teorema da Transitividade de Birkhoff (Teorema 1.17) que transitividade e hiperciclicidade são equivalentes. Usando tal teorema, exibimos uma demonstração do Critério de Hiperciclicidade. Com isso em mente, como vamos exibir um Critério de Sub-Hiperciclicidade é natural, então, que tentemos definir a noção de sub-transitividade.

Foi esse pensamento que levou os autores de [18] (os mesmos que enunciaram o Critério de Sub-Hiperciclicidade) a definirem a noção de sub-transitividade como apresentamos abaixo:

Definição 3.1 (Definição 3.2 de [18]). Sejam $X$ um espaço de Banach de dimensão infinita, $M$ um subespaço fechado de dimensão infinita tal que $M \neq X$ e $T \in \mathcal{B}(X)$. Dizemos que $T$ é subtransitivo com respeito a $\mathbf{M}$ se, para todo par $U, V \subseteq M$ de abertos de $M$, existir um $n \in \mathbb{N}$ tal que $T^{-n}(U) \cap V$ contém um subconjunto não-vazio que é aberto em $M$.

Neste caso, também dizemos que T é M-transitivo.

Lembrando do Teorema de Birkhoff é natural perguntar: será que $M$-transitividade é equivalente a $M$-hiperciclicidade? Em [18] os autores mostraram que $M$-transitividade implica em $M$ hiperciclicidade, como mostra o teorema abaixo: 
Teorema 3.2 (Teorema 3.5 de [18]). Seja X um espaço de Banach e $M$ um subespaço fechado de dimensão infinita e não-trivial. Se T é $M$-transitivo, então $T$ é $M$-hipercíclico.

E a outra implicação? Antes de responder a essa pergunta, vamos apresentar o Critério de SubHiperciclicidade. De maneira completamente análoga ao HC, o Critério de Sub-Hiperciclicidade nos fornece uma condição suficiente para que um operador seja $M$-hipercíclico:

Teorema 3.3 (Critério de Sub-Hiperciclicidade, Teorema 3.6 de [18]). Sejam $X$ um espaço de Banach separável de dimensão infinita, $T: X \rightarrow X$ um operador linear limitado e $M$ um subespaço fechado de dimensão infinita de $X$ tal que $M \neq X$. Suponha que existem subconjuntos $X_{0}, Y_{0}$ densos em $M$ e uma sequência crescente de inteiros positivos $\left(n_{k}\right)_{k \in \mathbb{N}}$ tais que:

(i) $T^{n_{k}}(x) \rightarrow 0$, para todo $x \in X_{0}$.

(ii) para cada $y \in Y_{0}$, existe uma sequência $\left(x_{k}\right)_{k \in \mathbb{N}} \subseteq M$ tal que

$$
x_{k} \rightarrow 0 \quad \text { e } \quad T^{n_{k}} x_{k} \rightarrow y
$$

(iii) $M$ é um subespaço invariante para $T^{n_{k}}$, para todo $k \in \mathbb{N}$

Então T é $M$-transitivo e, portanto, M-hipercíclico.

Observação 3.4. Apesar de não estar observado no artigo original, uma inspeção na demonstração do Critério de Sub-Hiperciclicidade (e de resultados anteriores relacionados) feita em [18], percebese que não se faz necessária a hipótese de que $X$ seja separável. De fato, como é de se esperar, podemos supor apenas que o subespaço $M$ em questão é separável.

Tomaremos, então, a liberdade de usar livremente esta versão "alternativa" (isto é, supondo $M$ separável e $X$ não necessariamente separável) no restante da tese.

Assim como no caso do HC, para facilitar a leitura tomaremos a liberdade de fazer referência ao Critério de Sub-Hiperciclicidade pela sigla SHC. Quando $T$ satisfazer o Critério de SubHiperciclicidade para um certo subespaço $M$, diremos que $T$ satisfaz o SHC para M.

Observe que se tomarmos a liberdade de escolher $M=X$, então o SHC se torna o HC, uma vez que a condição (iii) fica trivial. Além disso, se lembramos o que foi dito no começo do capítulo anterior (ver Observação 2.4), temos que hiperciclicidade e sub-hiperciclicidade são essencialmente equivalentes quando o subespaço em questão for invariante sob o operador dado. Isto nos leva ao seguinte lema:

Lema 3.5. Sejam $X$ um espaço de Banach de dimensão infinita, $T: X \rightarrow X$ um operador linear limitado e $M$ um subespaço fechado separável de dimensão infinita de $X$ tal que $M \neq X$. Suponha que $T$ seja $M$-invariante e considere o operador $\left.T\right|_{M}: M \rightarrow M$. Então $\left.T\right|_{M}$ satisfaz o HC se, e somente se, T satisfaz o SHC para $M$.

DemonstraÇÃo: Trivial. 
Com esse lema, podemos voltar à demonstração do Teorema 2.30 e verificar que, naquela demonstração, mostramos que o operador $\left.T\right|_{M}$ era mixing, uma vez que ele era quasi-conjugado com o operador mixing $S \in \mathcal{B}\left(\ell_{1}\right)$. Logo, sendo $\left.T\right|_{M}$ mixing, temos que ele satisfaz o Critério de Hiperciclicidade. Segundo o lema acima, isto mostra que o operador $T$ satisfaz o SHC para $M$. Não é difícil ver que tal raciocínio também se aplica para o Teorema 2.32, mostrando o seguinte teorema:

Teorema 3.6. Seja $X$ um espaço de Banach dimensão infinita. Seja $M \neq X$ um subespaço fechado separável de dimensão infinita. Então existe $T \in \mathcal{B}(X)$ tal que $T$ satisfaz o Critério de Sub-Hiperciclicidade para $M$.

Dito tudo isso, podemos então dizer que o SHC é quase um "análogo" do HC para subespaços. No entanto, para que o SHC seja um "análogo ideal" do HC para subespaços, devemos ter uma equivalência entre $M$-transitividade e $M$-hiperciclicidade, já que hiperciclicidade e transitividade são equivalenteł ${ }^{1}$ e, no caso do SHC, estamos demonstrado que o operador em questão é $M$-transitivo (assim como foi feito no caso do HC).

No entanto, em [17] Can Le mostrou um exemplo de um operador que é $M$-hipercíclico mas não é $M$-transitivo. Tal operador, portanto, não satisfaz o SHC - e mostra, claro, que $M$-hiperciclicidade não implica em $M$-transitividade, respondendo a pergunta que levantamos anteriormente. Além disso, no mesmo artigo Can Le mostrou que as condições (i) e (ii) do SHC não são suficientes para mostrar que um operador é $M$-hipercíclico.

Em [16] e [18 foram dados exemplos de operadores $T$ tal que $T$ é $M$-hipercíclico e $M$ não é invariante para $T^{n}$, para todo $n \in \mathbb{N}$. Levando em consideração o item (iii), temos que tais operadores também não satisfazem o SHC.

Uma pergunta que se manifesta é: seja $T$ um operador hipercíclico que satisfaz o HC. Ele satisfaz o SHC? Amplamente baseado no exemplo citado acima e apresentado em [16], temos o exemplo a seguir. Vale ressaltar que os exemplos citados anteriormente que foram dados em [17] e [18] também respondem a questão apresentada 2 No que segue, vamos denotar a $n$-ésima coordenada de um vetor $x_{k}$ por $x_{k}(n)$.

Exemplo 3.7 (baseado no Exemplo 2 de [16]). Seja $T:=2 B$, onde $B$ é o backward shift em $\ell_{2}$. Como vimos no Exemplo 1.18, $T$ é um operador hipercíclico e satisfaz o Critério de Hiperciclicidade. Pelo Corolário 2.7, segue que $T$ é sub-hipercíclico. Vamos mostrar que existe um subespaço fechado de dimensão infinita $M \subseteq \ell_{2}$ tal que $T$ é $M$-hipercíclico, $T^{n}(M) \nsubseteq M$ e $M \nsubseteq T^{n}(M)$. Tendo em vista a condição (iii) do Critério de Sub-Hiperciclicidade, isto mostra que $T$ não satisfaz o SHC.

Seja

$$
A:=\left\{k \in \mathbb{N}: \text { existe } n \in \mathbb{N} \text { tal que } 2^{n}-2<k \leq 3 \cdot 2^{n-1}-2\right\}
$$

Para exemplificar, temos que $A=\{1,3,4,7,8,9,10,15,16, \ldots, 21,22,31,32, \ldots, 45,46,63, \ldots\}$. Agora tome

$$
M=\left\{x=(x(k))_{k \in \mathbb{N}} \in \ell_{2}: x(k)=0 \text { se } k \in A\right\}
$$

\footnotetext{
${ }^{1}$ Vide o Teorema 1.17

${ }^{2}$ A técnica utilizada no exemplo dado em [18] é bastante similar à técnica utilizada no exemplo apresentado no texto. Não só isso, mas os dois exemplos também se utilizam do mesmo operador e espaço: o backward shift em $\ell_{2}$.
} 
Note que $M$ é claramente fechado e tem dimensão infinta. Seja $\left(B_{j}\right)_{j \in \mathbb{N}}$ uma sequência crescente de subconjuntos de $A^{c}$ dados por

$$
B_{j}=A^{c} \cap\left[1,2^{j+1}-2\right]
$$

para todo $j \in \mathbb{N}$.

Vamos mostrar agora que existem vetores $\left(y_{j}\right)_{j \in \mathbb{N}} \subseteq M$ tais que

(i) $\overline{\left\{y_{j}: j \in \mathbb{N}\right\}}=M$.

(ii) $y_{j}(k) \neq 0$ se, e somente se, $k \in B_{j}$.

(iii) $\left|y_{j}(k)\right| \leq j$, para todo $k, j \in \mathbb{N}$.

Seja $\left\{z_{j}: j \in \mathbb{N}\right\}$ um conjunto enumerável e denso em $M$. Para cada $j \in \mathbb{N}$, defina cada $x_{j}$ como

$$
x_{j}(k)=\left\{\begin{array}{l}
z_{j}(k), \text { se } k \in B_{j} \text { e }\left|z_{j}(k)\right| \leq j . \\
0, \text { caso contrário. }
\end{array}\right.
$$

Em primeiro lugar, é claro que $\left.x_{j} \in M \subseteq \ell_{2}\right]^{3}$ Mais ainda, temos que $\left\{x_{j}: j \in \mathbb{N}\right\}$ também é um conjunto enumerável e denso em $M$. De fato, seja $x=(x(k))_{k \in \mathbb{N}} \in M$ e $0<\varepsilon<1$. Fixe $j_{0} \in \mathbb{N}$ tal que $\sum_{k \geq j_{0}}|x(k)|^{2}<\varepsilon^{2} / 2$. Seja $\alpha:=\max \left\{|x(k)|: k<j_{0}\right\}$. Como $\left\{z_{j}: j \in \mathbb{N}\right\}$ é denso em $M$, podemos tomar $j_{1}>2^{j_{0}}+\alpha$ tal que $\left\|z_{j_{1}}-x\right\|<\varepsilon / \sqrt{2}$.

Tome um $k \in \mathbb{N}$ qualquer. Se $k \notin B_{j_{1}}$, pela definição de $B_{j_{1}}$ segue que $k \in A$ ou $k \geq 2^{j_{1}+1}-1$. Se $k \in A$, então $x(k)=0$. Se $k \geq 2^{j_{1}+1}-1$, então $k \geq j_{0}$ uma vez que $k \geq j_{1}>2^{j_{0}}+\alpha>j_{0}$.

Ainda, temos que $\left|z_{j_{1}}(k)\right|>j_{1}$ também implica que $k \geq j_{0}$; caso contrário teríamos que $|x(k)| \leq$ $\alpha$ (vide a definição de $\alpha$ ) e portanto:

$$
\left\|z_{j_{1}}-x\right\| \geq\left|z_{j_{1}}(k)-x(k)\right| \geq\left|z_{j_{1}}(k)\right|-|x(k)|>\left|z_{j_{1}}(k)\right|-\alpha>j_{1}-\alpha>2^{j_{0}}
$$

o que é uma contradição com a escolha de $z_{j_{1}}$.

Portanto, se $k \notin B_{j}$ ou $\left|z_{j_{1}}(k)\right|>j_{1}$, segue então que $k \geq j_{0}$. Uma vez que concluímos isso, temos então que:

$$
\begin{aligned}
\left\|x_{j_{1}}-x\right\|^{2} & =\sum_{k \in B_{j_{1}}} \text { e }\left|z_{j_{1}}(k)\right| \leq j_{1} \\
& \leq \| z_{j_{1}}(k)-\left.x(k)\right|^{2}+\sum_{k \notin B_{j_{1}} \text { ou }\left|z_{j_{1}}(k)\right|>j_{1}}|x(k)|^{2}+\sum_{k \geq j_{0}}|x(k)|^{2}<\varepsilon^{2} / 2+\varepsilon^{2} / 2 \\
& <\varepsilon^{2}
\end{aligned}
$$

o que mostra que $\left\{x_{j}: j \in \mathbb{N}\right\}$ é denso em $M$.

Observe que o conjunto $\left\{x_{j}: j \in \mathbb{N}\right\}$ satisfaz as condições (i) e (iii), mas não necessariamente satisfaz a condição (ii), uma vez que podemos ter $k \in B_{j}$ e $z_{j}(k)=0$. Daí, pela definição de $x_{j}$,

\footnotetext{
${ }^{3}$ De fato, observe que $x_{j}(k) \neq 0$ acontece apenas se $k \in B_{j}$ e $\left|z_{j}(k)\right| \leq j$. Agora, como $B_{j} \subseteq A^{c}$, isto quer dizer que $x_{j}(k)=0$ se $k \in A$. Por definição, segue que $x_{j} \in M$.

${ }^{4}$ Tecnicamente, se $k \notin B_{j_{1}}$ podemos ter o caso em que $k \in A$ e $k<2^{j_{1}+1}-1$. Como vimos, isto implica que $x(k)=0$ e, desta maneira, tal caso não fará diferença no cálculo a seguir.
} 
temos que $k \in B_{j}$ e $x_{j}(k)=0$, o que contradiz a condição (ii). De maneira a resolver isso, defina cada $y_{j}$ como

$$
y_{j}(k)=\left\{\begin{array}{l}
2^{-j k}, \text { se } k \in B_{j} \text { e }\left|x_{j}(k)\right|=0 . \\
x_{j}(k), \quad \text { caso contrário. }
\end{array}\right.
$$

Dessa forma as condições (i), (ii) e (iii) são satisfeitas.

Defina

$$
y=\sum_{j=1}^{\infty} S^{n_{j}} y_{j}
$$

onde $S:=2^{-1} F, F$ é o forward shift em $\ell_{2}, n_{j}:=\sum_{i=1}^{j} m_{i}$ e $m_{i}:=3 \cdot 2^{3 i}-2$, para $i, j \in \mathbb{N}$. Vamos mostrar agora que $y \in M$ e orb $(y, T) \cap M$ é denso em $M$.

Em primeiro lugar, para cada $j \in \mathbb{N}$, as posições não-nulas de $y_{j}$ estão contidos em um bloco de tamanho $2^{j+1}-2$ (vide a condição (ii)).

Afirmação. Todo vetor em $M$, a partir da posição $m_{j}+1=3 \cdot 2^{3 j}-1$, tem um bloco de, no máximo, $2^{3 j}$ posições não-nulas, para todo $j \in \mathbb{N}$.

De fato, note que pela definição de $A$, se $x \in M$ então $x\left(m_{j}\right)=0$. Logo, posições não-nulas (a partir de $m_{j}$ ) podem ir da posição $m_{j}+1=3 \cdot 2^{3 j}-1$ até $2^{3 j+2}-2$ (vide a definição de $M$, para $n=3 j+1)$. Daí, uma simples conta nos mostra que podemos ter $2^{3 j}$ posições não-nulas.

Agora, note que

$$
\begin{aligned}
n_{j-1} & =\sum_{i=1}^{j-1} m_{i}=\sum_{i=1}^{j-1}\left(3 \cdot 2^{3 i}-2\right)=3 \cdot\left(\sum_{i=1}^{j-1} 2^{3 i}\right)-2(j-1) \\
& =3 \cdot \frac{8\left(2^{3 j-3}-1\right)}{7}-(2 j-2)=\frac{3}{7} 2^{3 j}-\frac{3}{7} \cdot 8-2 j+2 \\
& =\frac{3}{7} 2^{3 j}-\frac{10}{7}-2 j
\end{aligned}
$$

e

$$
n_{j-1}+2^{j+1}-2=\frac{3}{7} 2^{3 j}-\frac{10}{7}-2 j+2^{j+1}-2<2^{3 j}
$$

Temos que $S^{n_{j}} y_{j} \in M$, para todo $j \in \mathbb{N}$. De fato, começando com $j=1$, uma vez que $B_{1}=A^{c} \cap[1,2]=\{2\}$, usando a condição (ii) temos que $y_{1}=(0, \lambda, 0,0, \ldots)$, com $|\lambda| \leq 15^{5}$ Como $n_{1}=m_{1}=22$, temos que $S^{n_{1}} y_{1}$ é tal que $S^{n_{1}} y_{1}(24)=\lambda$ e $S^{n_{1}} y_{1}(k)=0$, se $k \neq 24$. Tendo em vista que $24 \notin A$, pela definição de $M$ segue que $S^{n_{1}} y_{1} \in M$.

Suponha agora $j>1$. Vamos mostrar que todos as posições não-nulas de $S^{n_{j}} y_{j}$ estão em um bloco de tamanho $2^{3 j}$ (a partir de $m_{j}+1$ ). Daí, pela afirmação em destaque feita anteriormente, teremos que $S^{n_{j}} y_{j} \in M$.

Como $n_{j}=m_{j}+n_{j-1}$, então o vetor $S^{n_{j}} y_{j}$ tem somente zeros nas primeiras $m_{j}$ posições. Pela condição (ii), temos que $y_{j}(k) \neq 0$ se, e somente se, $k \in B_{j}=A^{c} \cap\left[1,2^{j+1}-2\right]$. Dessa forma, a partir da posição $m_{j}+1$, o vetor $S^{n_{j}} y_{j}$ tem, no máximo, $n_{j-1}+2^{j+1}-2$ posições não-nulas. Pela Equação (3.1), temos que $n_{j-1}+2^{j+1}-2<2^{3 j}$, como desejado.

\footnotetext{
${ }^{5}$ Vide a condição (iii).
} 
Não é difícil ver que $\left\|S^{n} x\right\|=2^{-n}\|x\|$. Com isso, segue das condições (ii), (iii) e da definição de $\left(n_{j}\right)_{j \in \mathbb{N}}$ que $\sum_{j=1}^{\infty} S^{n_{j}} y_{j}$ converge. Como $S^{n_{j}} y_{j} \in M$, para todo $j \in \mathbb{N}$, segue que $y \in M$. Resta mostrar que orb $(y, T) \cap M$ é denso em $M$. Para isso, vamos mostrar que $T^{n_{j}} \in M$ e $\left\|T^{n_{j}} y-y_{j}\right\|<2^{-j}$. Daí, como $\left\{y_{j}: j \in \mathbb{N}\right\}$ é denso em $M$, teremos o desejado.

Note que

$$
T^{n_{j}} y=T^{n_{j}}\left(\sum_{j=1}^{\infty} S^{n_{j}} y_{j}\right)=\sum_{n_{i}<n_{j}} T^{n_{j}-n_{i}} y_{i}+y_{j}+\sum_{n_{i}>n_{j}} S^{n_{i}-n_{j}} y_{i}
$$

uma vez que $T \circ S=I$.

Se $n_{i}<n_{j}$, então $n_{j}-n_{i} \geq m_{j}>2^{j+1}$. Portanto, como a maior posição não-nula de $y_{j}$ é, no máximo, $2^{j+1}-2$ (vide a condição (ii)), temos que $T^{n_{i}-n_{j}} y_{i}=0$, donde segue que

$$
\sum_{n_{i}<n_{j}} T^{n_{j}-n_{i}} y_{i}=0 \in M
$$

Por outro lado, se $n_{i}>n_{j}$, então $m_{i} \leq n_{i}-n_{j}<n_{i}$. Dessa forma, pelo mesmo argumento utilizado anteriormente, segue que cada vetor $S^{n_{i}-n_{j}} y_{i}$ tem suas posições não-nulas em um bloco de tamanho $2^{3 j}$ a partir da posição $m_{j}+1$. Portanto, temos que $S^{n_{i}-n_{j}} y_{i} \in M$ de onde segue que

$$
\sum_{n_{i}>n_{j}} S^{n_{i}-n_{j}} y_{i} \in M
$$

Como $y_{j} \in M$, então das equações em destaque temos que $T^{n_{j}} y$, como queríamos.

Tendo em mente as Equações (3.2) e (3.3), temos que:

$$
\left\|T^{n_{j}}-y_{j}\right\|=\left\|\sum_{n_{i}>n_{j}} S^{n_{i}-n_{j}} y_{i}\right\| \leq \sum_{n_{i}>n_{j}}\left\|S^{n_{i}-n_{j}} y_{i}\right\|
$$

Como já vimos, $\left\|S^{n} x\right\|=2^{-n}\|x\|$. Daí, usando as condições (ii) e (iii), temos que

$$
\left\|y_{i}\right\|^{2}=\sum_{k=1}^{\infty}\left|y_{i}(k)\right|^{2} \leq \sum_{k=1}^{2^{i+1}-2} i^{2}=\left(2^{i+1}-2\right) i^{2}
$$

Portanto:

$$
\sum_{n_{i}>n_{j}}\left\|S^{n_{i}-n_{j}} y_{i}\right\|=\sum_{i=j+1}^{\infty}\left\|S^{n_{i}-n_{j}} y_{i}\right\| \leq \sum_{i=j+1}^{\infty}\left(2^{-\left(n_{i}-n_{j}\right)}\right)\left\|y_{i}\right\| \leq \sum_{i=j+1}^{\infty} \frac{\sqrt{2^{i+1}-2} \cdot i}{2^{n_{i}-n_{j}}}
$$

Daí:

$$
\sum_{i=j+1}^{\infty} \frac{\sqrt{2^{i+1}-2} \cdot i}{2^{n_{i}-n_{j}}} \leq \sum_{i=j+1}^{\infty} \frac{\sqrt{2^{i+1}-2} \cdot i}{2^{m_{i}+m_{i-1}+\ldots+m_{j+1}}} \leq \sum_{i=j+1}^{\infty} \frac{\sqrt{2^{i+1}-2} \cdot i}{2^{2 i} \cdot 2^{m_{i-1}+\ldots+m_{j+1}}} \leq \sum_{i=j+1}^{\infty} \frac{1}{2^{i}}
$$


o que mostra o desejado 67

Para finalizar, note agora que o conjunto $A$ possui intervalos de tamanho arbitrário. Logo, pela definição de $M$, temos que $T^{n}(M) \nsubseteq M$. Da mesma maneira, $A^{c}$ também possui intervalos de tamanho arbitrário. Logo, $M \nsubseteq T^{n}(M)$, como desejado.

Vejamos agora mais um exemplo de um operador sub-hipercíclico que não satisfaz o SHC. Ao contrário do exemplo anterior, o espaço em questão será não-separável e, portanto, o operador não será hipercíclico. Dessa forma, podemos concluir (junto ao último exemplo) que satisfazer (ou não) o SHC pouco tem a ver com satisfazer (ou não) o HC. Veremos também que o argumento do exemplo a seguir será generalizado na Proposição 3.16 .

Exemplo 3.8. Seja $T \in \mathcal{B}\left(\ell_{1}\right)$ tal que $T$ não satisfaz o $H C$ (sabemos que tal operador existe pelo Teorema 1.19]. Como $\left(\ell_{1}\right)^{* *}=\left(\ell_{\infty}\right)^{*}$, considere $T^{* *} \in \mathcal{B}\left(\left(\ell_{\infty}\right)^{*}\right)$. É claro que $\left(\ell_{\infty}\right)^{*}$ não é separável uma vez que, se fosse, então teríamos que $\ell_{\infty}$ é separável 8 Logo, $T^{* *}$ não é um operador hipercíclico.

Como $\ell_{1}$ não é reflexivo, tomando por $i: \ell_{1} \rightarrow\left(\ell_{\infty}\right)^{*}$ a imersão canônica, temos pelo Corolário 2.28 que $T^{* *}$ é um operador $i\left(\ell_{1}\right)$-hipercíclico $9^{9}$ Vamos mostrar agora que ele não satisfaz o SHC para $i\left(\ell_{1}\right)$.

Lembrando o que fizemos na demonstração do Corolário 2.26, temos que $T^{* *} \circ i=i \circ T$. Logo, se $x \in \ell_{1}$, então $T^{* *}(i(x))=i(T x) \in i\left(\ell_{1}\right)$, o que mostra que $T^{* *}$ é $i\left(\ell_{1}\right)$-invariante. Podemos considerar, então, o operador $\left.T^{* *}\right|_{i\left(\ell_{1}\right)}: i\left(\ell_{1}\right) \rightarrow i\left(\ell_{1}\right)$.

Cometendo um pequeno abuso de notação (de maneira a simplificar o que segue), podemos também considerar $i: \ell_{1} \rightarrow i\left(\ell_{1}\right)$. Dessa forma, temos que $i$ é um operador invertível. Se $i^{-1}$ denota o seu inverso, então de $T^{* *} \circ i=i \circ T$ podemos concluir que

$$
\left.i^{-1} \circ T^{* *}\right|_{i\left(\ell_{1}\right)}=T \circ i^{-1}
$$

Suponha agora que $T^{* *}$ satisfaça o SHC para $i\left(\ell_{1}\right)$. Pelo Lema 3.5 temos então que $\left.T^{* *}\right|_{i\left(\ell_{1}\right)}$ satisfaz o HC. Pela última equação em destaque e a Proposição 1.26 , isto significa que $T$ satisfaz o HC, o que é uma contradição com a escolha de $T$.

Tendo em vista que temos vários exemplos de operadores sub-hipercíclicos que não satisfazem o SHC, Can Le demonstrou um segundo critério, que fornece uma condição suficiente para um operador ser $M$-hipercíclico (assim como o SHC) mas que não necessariamente é $M$-transitivo uma vez que, como já mostramos, estas duas propriedades não são equivalentes:

\footnotetext{
${ }^{6}$ Como $m_{i}=3 \cdot 2^{3 i}-2$, então não é difícil ver que $2^{m_{i}} \geq 2^{2 i}$.

${ }^{7}$ De fato, note que $\sqrt{2^{i+1}-2} \cdot i \leq \sqrt{2^{i+1}} \cdot i \leq 2^{i+1} \cdot i \leq 2^{i} \cdot 2 i \leq 2^{i} \cdot 2^{m_{i-1}} \leq 2^{i} \cdot 2^{m_{i-1}+\ldots+m_{j+1}}$. Daí, $\frac{\sqrt{2^{i+1}-2} \cdot i}{2^{i} \cdot 2^{m_{i}-1+\ldots+m_{j+1}}} \leq 1 \Rightarrow \frac{\sqrt{2^{i+1}-2} \cdot i}{2^{2 i} \cdot 2^{m_{i-1}+\ldots+m_{j+1}}} \leq \frac{1}{2^{i}}$.

${ }^{8}$ Ver [22, Teorema 1.12.11, p.112].

${ }^{9}$ De fato, temos que $\ell_{1}$ não é reflexivo pois $\ell_{1}$ é separável e $\left(\ell_{\infty}\right)^{*}$ não é o é; logo, não podem ser isomorfos. Vimos também no primeiro capítulo que $\left(\ell_{\infty}\right)^{*}=\ell_{1} \oplus c_{0}^{\perp}$, vide a Proposição 1.7 .
} 
Teorema 3.9 (Teorema 2.5 de [17]). Sejam X um espaço de Banach separável de dimensão infinita, $T: X \rightarrow X$ um operador linear limitado e $M$ um subespaço fechado de dimensão infinita de $X$ tal que $M \neq X$. Suponhamos que existem subconjuntos $X_{0}, Y_{0} \subseteq M$ tal que $Y_{0}$ é denso em $M$ e uma sequência crescente de inteiros positivos $\left(n_{k}\right)_{k \in \mathbb{N}}$ tais que:

(i) $T^{n_{k}}(x) \rightarrow 0$, para todo $x \in X_{0}$.

(ii) para cada $y \in Y_{0}$, existe uma sequência $\left(x_{k}\right)_{k \in \mathbb{N}} \subseteq X_{0}$ tal que

$$
x_{k} \rightarrow 0 \quad \text { e } \quad T^{n_{k}} x_{k} \rightarrow y
$$

(iii) $X_{0} \subseteq \cap_{k=1}^{\infty} T^{-n_{k}}(M)$.

Então T é M-hipercíclico.

Observação 3.10. Analogamente à Observação 3.4, uma inspeção na demonstração feita por Le mostra que a hipótese de $X$ ser separável não se faz totalmente necessária; apenas o subespaço $M$ em questão precisa ser separável.

Observe que o conjunto $X_{0}$ não precisa ser denso em $M$ (ao contrário do conjunto $X_{0}$ no SHC). Além disso, se vale a condição (iii) do SHC (isto é, $M$ é um subespaço invariante para $T^{n_{k}}$, para todo $k \in \mathbb{N}$ ), então $T^{n_{k}}(M) \subseteq M$, para todo $k \in \mathbb{N} \Rightarrow M \subseteq T^{-n_{k}}(M)$, para todo $k \in \mathbb{N} \Rightarrow X \subseteq$ $M \subseteq \cap_{k=1}^{\infty} T^{-n_{k}}(M)$, o que mostra que a condição (iii) do teorema anterior vale.

Ou seja, como esperado, se um operador $T$ satisfaz o SHC, então ele satisfaz o teorema anterior. No entanto, como Can Le mostra no Lema 2.8 e na Proposição 2.10 em [17], o contrário não vale.

Por fim, em [17] Can Le também apresentou um outro critério para sub-hiperciclicidade, bem diferente dos que já foram apresentados. Este é o assunto da próxima seção.

\subsection{Um Outro Critério}

Antes de mostrar o critério, precisamos de uma definição:

Definição 3.11. Dado um operador linear $T$, vamos definir como o núcleo generalizado de $T o$ conjunto

$$
\operatorname{ker}^{*}(T):=\bigcup_{m=1}^{\infty} \operatorname{ker}\left(T^{m}\right)
$$

Com esta definição em mãos, temos o critério apresentado por Le:

Teorema 3.12 (Teorema 3.1 de [17]). Sejam $X$ um espaço de Banach separável e $T \in \mathcal{B}(X)$ tal que $\operatorname{ker}^{*}(T)$ é denso em $X$. Suponha também que exista uma função $A: \operatorname{ker}^{*}(T) \rightarrow \operatorname{ker}^{*}(T)$ tal que:

(1) $A^{m} x \rightarrow 0$ para todo $x \in \operatorname{ker}^{*}(T)$.

(2) $T \circ A=I$ em $\operatorname{ker}^{*}(T)$. 
Então $T$ é $M$-hipercíclico para todos os subespaços $M$ de codimensão finita em $X$.

Como Le nota em seu artigo, um operador que satisfaz as condições (1) e (2) satisfaz, necessariamente, o Critério de Hiperciclicidade. De fato, tomando $X_{0}=Y_{0}=\operatorname{ker}^{*}(T)$, então $T^{m} x \rightarrow 0$, satisfazendo a condição (i) do Critério. Definindo $S_{m}$ por $A^{m}$, então por (1) temos que $S_{m}$ satisfaz o (ii) do HC. Por fim, usando a condição (2) do teorema acima, é imediato ver que a condição (iii) do Critério é satisfeita, mostrando que $T$ satisfaz o HC.

Antes de prosseguirmos, vamos destacar uma observação que será importante ainda nesta seção:

Observação 3.13. Assim como observado em resultados anteriores, a hipótese do espaço $X$ ser separável no Teorema 3.12 pode ser substituída se modificarmos ligeiramente o seu enunciado. Para isso, tome $X$ um espaço de Banach qualquer, $S \in \mathcal{B}(X)$ e $M \subseteq X$ um subespaço fechado e separável. Suponha agora que $\operatorname{ker}^{*}(S) \subseteq M$ é denso em $M$. Dessa forma, temos que $S$ é $M$-invariante. De fato, se $m \in M$ e $\operatorname{ker}^{*}(S) \subseteq M$ é denso em $M$, então existe $\left(x_{n}\right)_{n \in \mathbb{N}} \in \operatorname{ker}^{*}(S)$ tal que $x_{n} \rightarrow m$. Como $S\left(x_{n}\right) \in \operatorname{ker}^{*}(S)$, para todo $n \in \mathbb{N}$, e $S\left(x_{n}\right) \rightarrow S(m)$ então $S(m) \in \overline{\operatorname{ker}^{*}(S)}=M$.

Suponha agora que exista $A_{1}: \operatorname{ker}^{*}(S) \rightarrow \operatorname{ker}^{*}(S)$ tal que $A_{1}$ satisfaz as condições $(1)$ e (2) do Teorema 3.12. Como $S$ é $M$-invariante, o operador $\left.S\right|_{M}: M \rightarrow M$ está bem definido. Logo, tomando no enunciado do Teorema $3.12 X:=M, T:=\left.S\right|_{M}$ e $A:=A_{1}$, então temos que $T=\left.S\right|_{M}$ é $N$-hipercíclico para todos os subespaços $N$ de codimensão finita em $M{ }^{10}$

Apesar de $M$ obviamente possuir codimensão finita em $M$, vamos tomar o cuidado de mostrar que se $T=\left.S\right|_{M}$ também é $M$-hipercíclico ${ }^{11}$ Nas condições do parágrafo anterior, temos que $T$ é $M$-invariante. Logo, considerando o operador $\left.T\right|_{M}$ em $M$, as condições (1) e (2) implicam que $\left.T\right|_{M}$ satisfaz o HC (de maneira semelhante ao que mostramos antes desta observação) e, portanto, $\left.T\right|_{M}$ é hipercíclico em $M$ - ou seja, $T$ é $M$-hipercíclico.

Em resumo, para generalizarmos o teorema anterior para espaços de Banach não-separáveis, basta supormos que existe um subespaço fechado, separável e de dimensão infinita $M$ tal que $\operatorname{ker}^{*}(T) \subseteq M$ é denso em $M$. Assim, se as condições (1) e (2) do Teorema 3.12 forem satisfeitas para tal $\operatorname{ker}^{*}(T) \subseteq M$, então $T$ será $N$-hipercíclico para todos os subespaços $N$ de codimensão finita em $M$ (e, como mostramos na observação anterior, $T$ também será $M$-hipercíclico).

Considere agora o espaço de Hardy-Hilbert $\mathbf{H}^{2}$. ${ }^{12}$ Em [21] os autores conseguiram mostrar que um certo operador em $\mathbf{H}^{2}$ satisfaz as condições do teorema anterior e, portanto, era $M$-hipercíclico para todos os subespaços $M$ de codimensão finita em $\mathbf{H}^{2}$. Com isso, como vimos que todo operador que satisfaz o Teorema 3.12 satisfaz o HC, lembrando do Teorema 1.19 segue que o resultado de Le não é redundante.

\footnotetext{
${ }^{10}$ Como veremos a seguir, podemos concluir também que $T$ é $M$-hipercíclico.

${ }^{11}$ Apenas uma tecnicalidade, uma vez que claramente $M$ também tem codimensão finita em $M$; no entanto, na demonstração original do Teorema 3.12 Le não considera subespaços de codimensão finita igual a zero em $X$ - a saber, o próprio $X$, uma vez que, como acabamos de ver, o operador que satisfaz as hipóteses de tal teorema já é hipercíclico.

${ }^{12} \mathrm{O}$ espaço de Hardy-Hilbert $\mathbf{H}^{2}$ é o conjunto de todas as funções analíticas no disco unitário tais que a sequência formada pelos coeficientes de sua série de Taylor pertence a $\ell_{2}$. A norma de uma função $f \in \mathbf{H}^{2}$ é dada pela norma $\left(\right.$ em $\left.\ell_{2}\right)$ de tal sequência formada pelos coeficientes. Este espaço é um espaço de Hilbert. Para mais detalhes, ver [20].
} 
$\mathrm{Na}$ tentativa de criar um resultado que garanta a existência de operadores sub-hipercíclicos em espaços de Banach quaisquer, começamos a estudar a técnica utilizada no Exemplo $3.7{ }^{13}$ Acabamos por perceber que, se tentarmos utilizar a mesma técnica usada em tal exemplo junto a hipóteses parecidas com as que Le fez em seu teorema, poderíamos obter um outro resultado:

Teorema 3.14. Sejam $X$ um espaço de Banach e $T \in \mathcal{B}(X)$. Suponha que exista um subespaço fechado e separável $M$ tal que $\operatorname{ker}^{*}(T) \cap M$ é denso em $M$. Suponha também que exista uma função $A: \operatorname{ker}^{*}(T) \rightarrow \operatorname{ker}^{*}(T)$ e uma sequência crescente $\left(m_{k}\right)_{k \in \mathbb{N}} \subseteq \mathbb{N}$ tal que:

(i) $A^{m_{k}} x \rightarrow 0$ para todo $x \in \operatorname{ker}^{*}(T) \cap M$.

(ii) $A^{m_{k}} x \in \operatorname{ker}^{*}(T) \cap M$, para todo $x \in \operatorname{ker}^{*}(T) \cap M$.

(iii) $m_{j}-m_{i} \in\left(m_{k}\right)_{k \in \mathbb{N}}$, para todo $i<j$.

(iv) $T \circ A=I$ em $\operatorname{ker}^{*}(T) \cap M$.

Então T é M-hipercíclico.

DemonstraÇão: Seja $\left(x_{k}\right)_{k \in \mathbb{N}} \subseteq \operatorname{ker}^{*}(T) \cap M$ uma sequência densa em $M$.

Afirmação. Existe uma subsequência crescente $\left(m_{j_{k}}\right)_{k \in \mathbb{N}} \subseteq\left(m_{k}\right)_{k \in \mathbb{N}}$ tal que

$$
\left\|A^{m_{j_{k}}} x_{k}\right\|<2^{-k}, \quad\left\|A^{m_{j_{k}+i}} x_{k+1}\right\|<2^{-(k+1)}, \quad T^{m_{j_{k}}} x_{k}=0 \quad \text { e } \frac{m_{j_{k+1}}}{m_{j_{k}}} \geq 2
$$

para todo $i \in \mathbb{N}_{0}$.

Não é difícil ver que é possível tomarmos tal sequência. De maneira a deixar a demonstração completa, vamos mostrar essa afirmação. Comecemos com $k=1$. Dado $\varepsilon=2^{-1}$, pela condição (i) existem $m_{k_{1}}, m_{k_{1}^{*}}$ tais que $\left\|A^{m_{j}} x_{1}\right\|<2^{-1}$, para todo $j \geq k_{1}$ e $\left\|A^{m_{j}} x_{2}\right\|<2^{-2}$, para todo $j \geq k_{1}^{*}$. Como $x_{1} \in \operatorname{ker}^{*}(T)$, então existe $p_{1}$ tal que $x_{1} \in \operatorname{ker}\left(T^{p_{1}}\right)$. Tomando $m_{j_{1}} \in\left(m_{k}\right)_{k \in \mathbb{N}}$ tal que $m_{j_{1}} \geq \max \left\{m_{k_{1}}, m_{k_{1}^{*}}, p_{1}\right\}$, é claro que vale que $\left\|A^{m_{j_{1}}} x_{1}\right\|<2^{-1},\left\|A^{m_{j_{1}+i}} x_{2}\right\|<2^{-2}$ e $T^{m_{j_{1}}} x_{1}=0$.

Suponha agora que já obtemos $\left(m_{j_{k}}\right)_{k=1}^{t-1}$. Assim como o parágrafo anterior, podemos obter $m_{k_{t}}, m_{k_{t}^{*}}$ tais que $\left\|A^{m_{j}} x_{t}\right\|<2^{-t}$, para todo $j \geq k_{t}$ e $\left\|A^{m_{j}} x_{t+1}\right\|<2^{-(t+1)}$, para todo $j \geq k_{t}^{*}$. Ainda, temos também que existe $p_{t}$ tal que $x_{t} \in \operatorname{ker}\left(T^{p_{t}}\right)$. Tomando agora $m_{j_{t}} \in\left(m_{k}\right)_{k \in \mathbb{N}}$ tal que $m_{j_{t}} \geq \max \left\{m_{k_{t}}, m_{k_{t}^{*}}, p_{t}, 2 m_{j_{t-1}}\right\}$, temos que $m_{j_{t}}$ satisfaz as quatro condições descritas na afirmação em destaque.

Com essa sequência $\left(m_{j_{k}}\right)_{k \in \mathbb{N}}$ em mãos, observe agora que $\sum_{k=1}^{\infty} A^{m_{j_{k}}} x_{k}$ converge absolutamente. De fato, uma vez que $\left\|A^{m_{j_{k}}} x_{k}\right\|<2^{-k}$ para todo $k \in \mathbb{N}$, então $\sum_{k=1}^{\infty}\left\|A^{m_{j_{k}}} x_{k}\right\|$ converge. Como $X$ é um espaço de Banach, segue que $\sum_{k=1}^{\infty} A^{m_{j_{k}}} x_{k}$ converge.

Defina então $x:=\sum_{k=1}^{\infty} A^{m_{j_{k}}} x_{k}$. Em primeiro lugar, observe que $x \in M$, uma vez que $A^{m_{j_{k}}} x_{k} \in$ $\operatorname{ker}^{*}(T) \cap M$ (vide a condição (ii)), $\operatorname{ker}^{*}(T) \cap M$ é um subespaço e $\overline{\operatorname{ker}^{*}(T) \cap M}=M$. Vamos agora mostrar que $T^{m_{j_{k}} x} \in M$ e $\left\{T^{m_{j_{k}} x}: k \in \mathbb{N}\right\}$ é denso em $M$. Isto claramente mostra que $T$ é $M$-hipercíclico, como desejamos.

- $T^{m_{j_{k}} x} \in M$.

\footnotetext{
${ }^{13}$ Este estudo culminou, além do próximo resultado, nos já vistos Teorema 2.30 e Teorema 2.32
} 
Usando a condição (iv), temos que

$$
T^{m_{j_{k}}} x=\sum_{i=1}^{k-1} T^{m_{j_{k}}-m_{j_{i}}} x_{i}+x_{k}+\sum_{i=k+1}^{\infty} A^{m_{j_{i}}-m_{j_{k}}} x_{i}
$$

Provemos agora que cada uma das parcelas pertence a $M$. Dessa forma, teremos que $T^{m_{j_{k}}} x \in$ $M$. Para a primeira parcela, fixe um $i \in\{1, \ldots, k-1\}$. Como $m_{j_{k+1}} / m_{j_{k}} \geq 2$, então é claro que $m_{j_{k}}-m_{j_{i}} \geq m_{j_{i}}$. Logo, de $T^{m_{j_{i}}} x_{i}=0$ (vide a construção da subsequência $\left(m_{j_{k}}\right)_{k \in \mathbb{N}}$ ), segue que $T^{m_{j_{k}}-m_{j_{i}}} x_{i}=0$ e, portanto,

$$
\sum_{i=1}^{k-1} T^{m_{j_{k}}-m_{j_{i}}} x_{i}=0
$$

É claro que $x_{k} \in M$. Agora, para $i>k$, pela condição (iii) temos que $m_{j_{i}}-m_{j_{k}} \in\left(m_{k}\right)_{k \in \mathbb{N}}$. Segue portanto, desta vez pela condição (ii), que $A^{m_{j_{i}}-m j_{k}} x_{i} \in \operatorname{ker}^{*}(T) \cap M$. Daí, temos que

$$
\sum_{i=k+1}^{\infty} A^{m_{j_{i}}-m_{j_{k}}} x_{i} \in \overline{\operatorname{ker}^{*}(T) \cap M}=M
$$

como esperado.

- $\left\{T^{m_{j_{k}} x}: k \in \mathbb{N}\right\}$ é denso em $M$.

Vamos mostrar que $\left\|T^{m_{j_{k}}} x-x_{k}\right\|<2^{-k}$. Como $\left(x_{k}\right)_{k \in \mathbb{N}}$ é denso em $M$, seguirá o desejado. Da Equação (3.4), temos:

$$
\begin{aligned}
\left\|T^{m_{j_{k}}} x-x_{k}\right\| & =\left\|\sum_{i=1}^{k-1} T^{m_{j_{k}}-m_{j_{i}}} x_{i}+\sum_{i=k+1}^{\infty} A^{m_{j_{i}}-m_{j_{k}}} x_{i}\right\| \\
& \leq \sum_{i=1}^{k-1}\left\|T^{m_{j_{k}}-m_{j_{i}}} x_{i}\right\|+\sum_{i=k+1}^{\infty}\left\|A^{m_{j_{i}}-m_{j_{k}}} x_{i}\right\|
\end{aligned}
$$

Como vimos anteriormente, temos que $\sum_{i=1}^{k-1} T^{m_{j_{k}}-m_{j_{i}}} x_{i}=0$. Fixemos agora um $i>k$. Então $m_{j_{i}}>m_{j_{i-1}} \geq m_{j_{k}}$. Como $m_{j_{k+1}} / m_{j_{k}} \geq 2$, para todo $k \in \mathbb{N}$, temos então que

$$
m_{j_{i}}-m_{j_{k}} \geq m_{j_{i}}-m_{j_{i-1}} \geq m_{j_{i-1}}
$$

Pela condição (iii) temos que $m_{j_{i}}-m_{j_{k}} \in\left(m_{k}\right)_{k \in \mathbb{N}}$. Logo, se denotarmos $m_{j_{i}}-m_{j_{k}}=m_{l}$, da desigualdade em destaque acima obtemos que $m_{l}>m_{j_{i-1}}$; ou seja, $l>j_{i-1}$. Logo, existe um $a_{i} \in \mathbb{N}$ tal que $l=j_{i-1}+a_{i}$, donde segue que

$$
\left\|A^{m_{j_{i}}-m_{j_{k}}} x_{i}\right\|=\left\|A^{m_{l}} x_{i}\right\|=\left\|A^{m_{j_{i-1}+a_{i}}} x_{i}\right\|
$$

Daí, por construção, temos que

$$
\left\|A^{m_{j_{i-1}+a_{i}}} x_{i}\right\|<2^{-i}
$$

ou seja, $\left\|A^{m_{j_{i}}-m_{j_{k}}} x_{i}\right\|<2^{-i}$. Como $i>k$ era qualquer, então: 


$$
\begin{aligned}
\left\|T^{m_{j_{k}}} x-x_{k}\right\| & \leq \sum_{i=1}^{k-1}\left\|T^{m_{j_{k}}-m_{j_{i}}} x_{i}\right\|+\sum_{i=k+1}^{\infty}\left\|A^{m_{j_{i}}-m_{j_{k}}} x_{i}\right\| \\
& \leq \sum_{i=k+1}^{\infty} 2^{-i}=2^{-k}
\end{aligned}
$$

como queríamos.

Fica claro, como dissemos, que a técnica utilizada no teorema acima e no Exemplo 3.7 é, em grande parte, a mesma. Le, em seu teorema apresentado anteriormente, utiliza uma técnica bastante semelhante - ainda que com algumas modificações devido a algumas diferenças nas hipóteses e a natureza de sua tese (mostrar que $T$ é $M$-hipercíclico para subespaços $M$ de codimensão finita) que é diferente do que obtemos no resultado anterior (mostramos que $T$ é $M$-hipercíclico, onde $M$ é tal que $\operatorname{ker}^{*}(T) \cap M$ é denso em $\left.M\right)$.

Observe que estamos supondo no teorema anterior que $X$ é um espaço de Banach qualquer (não necessariamente separável), enquanto no resultado de Le o espaço em questão é separável. Com isso, se um operador $T$ satisfazer as condições (i) a (iv) de nosso resultado, então o operador não necessariamente satisfaz o HC - uma vez que o espaço $X$ utilizado pode não ser separável ao contrário do resultado de Le, como comentamos anteriormente. Sendo rigoroso, podemos dizer então que o Teorema 3.14 é mais geral que o Teorema 3.12 .

No entanto, seguindo o que dissemos na Observação 3.13. é possível retirar a hipótese de $X$ ser separável no Teorema 3.12 e obter uma versão "estendida" deste resultado para espaços nãoseparáveis - com isso em mente, a partir de agora teremos em mente esta versão "estendida" ao fazermos menção ao Terema 3.12 .

Logo, pensando em espaços não necessariamente separáveis, tanto o Teorema 3.12 quanto o Teorema 3.14 nos permitem concluir que $T$ é $M$-hipercíclico. Ainda, o Teorema 3.12 também nos permite concluir que $T$ é $N$-hipercíclico para todos os subespaços $N$ de codimensão finita em $M$ (como vimos na Observação 3.13). Surge, então, a pergunta natural: se as conclusões do Teorema 3.12 são melhores que as do Teorema 3.14, que vantagens este segundo teorema possui?

Para responder a essa pergunta, vale frisar que existem algumas sutis diferenças entre os dois resultados: a saber, as hipóteses sobre $\operatorname{ker}^{*}(T)$ e sobre $A$. Se $T$ e $A$ satisfazem a versão "estendida" do Teorema 3.12 (isto é, eles são tais que $\operatorname{ker}^{*}(T) \subseteq M$ é denso em $M$ e $A$ satifaz (1) e (2)), então não é difícil perceber que $T$ e $A$ também satisfazem as condições do Teorema 3.14 para a sequência $(k)_{k \in \mathbb{N}}$. Além disso, como dissemos na Observação 3.13 , a hipótese de que $\operatorname{ker}^{*}(T) \subseteq M$ é denso em $M$ implica que $T$ seja $M$-invariante, enquanto as hipóteses feitas no Teorema 3.14 não necessariamente tem essa consequência. Logo, embora a tese do Teorema 3.12 seja "melhor", as hipóteses feitas no Teorema 3.14 são mais gerais - e, portanto, mais operadores podem satisfazer tal resultado.

Observe ainda que o resultado de Le não pode ser estendido de uma maneira "simples" (isto é, a partir de hipóteses semelhantes obtermos uma conclusão semelhante) para espaços não-separáveis, 
uma vez que subespaços de codimensão finita em espaços não-separáveis são, evidentemente, nãoseparáveis - e tais subespaços claramente não admitem operadores sub-hipercíclicos.

De maneira a ilustrar os argumentos apresentados, vejamos um simples exemplo:

Exemplo 3.15. Seja $X=\ell_{\infty}$ e seja $T=2 B$, onde $B \in \mathcal{B}\left(\ell_{\infty}\right)$ é o conhecido backward shift (vide o Exemplo 1.18). Seja $M=\left\{\left(a_{n}\right): a_{n} \rightarrow 0\right.$ e $\left.a_{2 n}=0\right\}$. Note que $M$ é um subespaço fechado de $\ell_{\infty}$ e claramente separável (uma vez que $M \subseteq c_{0}$ ). Observe ainda que $T$ não é $M$-invariante, o que mostra que $T$ já não pode cumprir as hipóteses da versão "estendida" do Teorema 3.12 e, portanto, tal resultado não se aplica à este $T$ e $M$

Temos ainda que $\operatorname{ker}^{*}(T)=c_{00}$ e, portanto, $\operatorname{ker}^{*}(T) \nsubseteq \nsubseteq M$. Assim, temos mais um motivo pelo qual o Teorema 3.12 não se aplica. Por outro lado, temos que $\operatorname{ker}^{*}(T) \cap M$ é claramente denso em $M$. Seja agora $F$ o já visto forward shift em $\mathcal{B}\left(\ell_{\infty}\right)$. Tomando $A=2^{-1} F$ e $\left(m_{k}\right)_{k \in \mathbb{N}}=(2 k)_{k \in \mathbb{N}}$, temos que $A$ e $\left(m_{k}\right)_{k \in \mathbb{N}}$ satisfazem as quatro condições do Teorema 3.14 e, portanto, $T$ é $M$-hipercíclico, como desejado.

\subsection{Quasi-Conjugações}

Uma vez que vimos um Critério para Sub-Hiperciclicidade, vamos agora "estender" também os resultados conhecidos para o Critério de Hiperciclicidade e quasi-conjugações (vide, por exemplo, o item (ii) da Proposição 1.26), no mesmo espírito do que fizemos no capítulo anterior:

Proposição 3.16. Sejam $X, Y$ espaços de Banach de dimensão infinita e $T \in \mathcal{B}(X), S \in \mathcal{B}(Y)$. Seja $i: X \rightarrow Y$ um operador linear limitado tal que $i$ é injetor, tem imagem fechada, não é sobrejetor e satisfaz $S \circ i=i \circ T$. Então $T$ satisfaz o Critério de Hiperciclicidade se, e somente se, $S$ satisfaz o Critério de Sub-Hiperciclicidade para $i(X)$.

DemonstraÇÃo: Vamos mostrar primeiro a ida. Em primeiro lugar, observe que como $i$ é injetor, tem imagem fechada e não é sobrejetor, então $i(X)$ é um subespaço fechado, de dimensão infinita e tal que $i(X) \neq Y$.

Se $X_{0}, Y_{0}$ são os conjuntos densos em $X$ para os quais $T$ satisfaz o $\mathrm{HC}$, tomemos $i\left(X_{0}\right), i\left(Y_{0}\right)$ como sendo os conjuntos densos em $i(X)$ exigidos pelo SHC para $S$. Tome também a sequência $\left(n_{k}\right)_{k \in \mathbb{N}}$ para qual $T$ satisfaz o HC.

Então, se $i(x) \in i\left(X_{0}\right)$, temos que:

$$
S^{n_{k}} i(x)=i\left(T^{n_{k}} x\right) \rightarrow i(0)=0
$$

uma vez que $T^{n_{k}} x \rightarrow 0$, para todo $x \in X_{0}$, como diz o item (i) do HC. Logo, $S$ satisfaz o item (i) do Critério de Sub-Hiperciclicidade.

Seja agora $i(y) \in i\left(Y_{0}\right)$. Logo, como $T$ satisfaz o HC, então existe uma sequência $\left(x_{k}\right)_{k \in \mathbb{N}} \subseteq X$ tal que $x_{k} \rightarrow 0$ e $T^{n_{k}} x_{k} \rightarrow y$ (vide a Observação 1.15. Daí, temos que $\left(i\left(x_{k}\right)\right)_{k \in \mathbb{N}} \subseteq i(X)$ é tal que $i\left(x_{k}\right) \rightarrow 0$ e $S^{n_{k}} i\left(x_{k}\right)=i\left(T^{n_{k}} x_{k}\right) \rightarrow i(y)$, como exige o item (ii) do Critério de Sub-Hiperciclicidade.

\footnotetext{
${ }^{14}$ Como já mostramos, se $T$ cumprisse as hipóteses do corolário, então $T$ seria $M$-invariante.
} 
Resta agora mostrar o item (iii). Se $i(x) \in i(X)$, então $S^{n_{k}} i(x)=i\left(T^{n_{k}} x\right) \in i(X)$, uma vez que $T^{n_{k}} x \in X$. Logo, $S$ satisfaz o Critério de Sub-Hiperciclicidade para $i(X)$, como desejado.

Para mostrar a outra implicação, vamos seguir o mesmo raciocínio do Exemplo 3.8. Observe que, como $S \circ i=i \circ T$, então $S$ é $i(X)$-invariante. Logo, podemos considerar o operador $\left.S\right|_{i(X)}$ : $i(X) \rightarrow i(X)$.

A partir de agora vamos cometer o abuso de considerar o contradomínio de $i$ como sendo sua imagem; isto é, vamos considerar $i$ como sendo o operador $i: X \rightarrow i(X)$. Sendo $i$ linear, limitado, injetor e com imagem fechada, ele é invertível. Denotando por $i^{-1}$ o seu inverso, então de $S \circ i=i \circ T$ obtemos que

$$
\left.i^{-1} \circ S\right|_{i(X)}=T \circ i^{-1}
$$

Se $S$ satisfaz o SHC para $i(X)$, então pelo Lema 3.5 temos que $\left.S\right|_{i(X)}$ satisfaz o HC. Pela equação acima e a Proposição 1.26 , isto implica que $T$ satisfaz o HC, como desejado.

Proposição 3.17. Sejam $X, Y$ espaços de Banach de dimensão infinita e $T \in \mathcal{B}(X), S \in \mathcal{B}(Y)$. Seja $i: X \rightarrow Y$ um operador linear limitado tal que $i$ é injetor, tem imagem fechada e satisfaz $S \circ i=i \circ T$. Se $M$ é um subespaço tal que $i(M) \neq Y$, então $T$ satisfaz o Critério de SubHiperciclicidade para $M$ se, e somente se, $S$ satisfaz o Critério de Sub-Hiperciclicidade para $i(M)$.

DEmonstraÇão: É completamente análogo à última proposição.

Mais uma vez, vale destacar que as hipóteses de $i$ não ser sobrejetor e $i(M) \neq Y$ foram colocadas, respectivamente, nas duas últimas proposições para evitar o caso em que $S$ satisfaz o SHC para $i(X)=i(M)=Y$, isto é, o caso em que $S \in \mathcal{B}(Y)$ satisfaz o HC. Tal caso já foi demonstrado na Proposição 1.26 .

Indo no espírito do Corolário 2.26 visto no capítulo anterior, temos o seguinte resultado:

Corolário 3.18. Sejam $X$ um espaço de Banach não-reflexivo e $T \in \mathcal{B}(X)$ um operador linear limitado. Denote por $i$ a imersão isométrica canônica $i: X \rightarrow X^{* *}$. Então:

(a) T satisfaz o Critério de Hiperciclicidade se, e somente se, $T^{* *}$ satisfaz o Critério de SubHiperciclicidade para $i(X)$.

(b) T satisfaz o Critério de Sub-Hiperciclicidade para $M$ se, e somente se, $T^{* *}$ satisfaz o Critério de Sub-Hiperciclicidade para $i(M)$.

Demonstração: Imediato das duas últimas proposições. 


\section{Capítulo 4}

\section{Ciclicidade e Hiperciclicidade}

Como constatamos no primeiro capítulo, existe uma clara relação entre hiperciclicidade e ciclicidade. Tal relação pode ser surpreendente em alguns momentos. Por exemplo, o Teorema 1.20 afirma que quando $T$ é hipercíclico, ciclicidade e hiperciclicidade são equivalentes para o operador $T \oplus T$.

Neste capítulo vamos explorar mais afundo a relação entre ciclicidade e hiperciclicidade. Um dos motivos para estudarmos tal relação se dá por conta do seguinte problema:

Problema 5. Existe um espaço de Banach $X$ tal que todo operador hipercíclico $T$ satisfaz o Critério de Hiperciclicidade?

A priori, tal problema parece não relacionar hiperciclicidade e ciclicidade de nenhuma maneira. Antes de elucidarmos como esta relação acontece, vale destacar que tal problema provém de questões que surgem naturalmente após estudarmos o Teorema 1.19. Por conta de tal teorema, sabemos que os espaços clássicos $c_{0}$ e $\ell_{p}(1 \leq p<\infty)$ possuem operadores hipercíclicos que não satisfazem o HC. Por serem espaços tão importantes na teoria de espaços de Banach, é natural perguntar se, afinal, existe algum espaço em que todo operador hipercíclico satisfaz o HC.

Para resolver o Problema 5 temos duas opções imediatas: construir um espaço de Banach separável que resolva o problema (como, por exemplo, Enflo e Gowers/Maurey fizeram para resolver problemas clássicos em espaços de Banach) ou achar um espaço separável conhecido e mostrar que ele soluciona o problema.

Escolhendo o segundo caminho, um espaço interessante a ser considerado como candidato a resolver o problema acima é o espaço de Argyros-Haydon $X_{A H}$ (construído em [3]). Ele é conhecido por ter sido o primeiro espaço de Banach construído com a propriedade de que todo operador $T \in \mathcal{B}\left(X_{A H}\right)$ pode ser escrito na forma $T=\lambda I+K$, onde $I$ é o operador identidade e $K$ é um operador compacto. Vamos destacar tal propriedade com a próxima definição:

Definição 4.1. Se $X$ é um espaço de Banach tal que todo operador $T \in \mathcal{B}(X)$ pode ser escrito na forma $T=\lambda I+K$, onde $I$ é o operador identidade e $K$ é um operador compacto, então dizemos que $X$ tem pouquíssimos operadores 1

Outros exemplos de espaços com pouquíssimos operadores são $X_{\mathfrak{n} r}$ e $X_{K u s}$ construídos, respectivamente, no preprint [4] e no artigo [19].

\footnotetext{
${ }^{1}$ Uma tradução livre do inglês, very few operators. Tal propriedade também é encontrada na literatura denominada scalar-plus-compact property.
} 
Não é difícil justificar o porquê de nos interessarmos em estudarmos hiperciclicidade em espaços com pouquíssimos operadores: estudar uma classe particular de operadores em um certo espaço (neste caso, operadores da forma $\lambda I+K$ no espaço $X_{A H}$ ) tende a ser mais vantajoso do que estudar operadores hipercíclicos sob condições mais gerais. Além disso, os operadores da forma $\lambda I+K$ são os operadores mais simples e naturais que um espaço de Banach pode ter. De fato, usando o Teorema de Hahn-Banach, obtemos uma vasta quantidade de operadores de posto finito 2 Como em espaços de Banach todo limite (quando existir) de operadores de posto finito é compacto, então os operadores compactos estão entre aqueles que aparecem naturalmente em espaços de Banach. Somando tais operadores compactos com operadores da forma $\lambda I, \lambda \in \mathbb{K}$ - outra classe de operadores que surge naturalmente no espaço - então obtemos os conhecidos operadores da forma $\lambda I+K$. Somada a essa naturalidade, temos também o fato de que não existem outras ferramentas que sejam igualmente gerais e que possibilitem construir operadores que não sejam da forma $\lambda I+K$.

Outro aspecto interessante e que nos motiva a estudar os operadores hipercíclicos em espaços com pouquíssimos operadores é o seguinte: não é conhecido ainda se, em geral, operadores hipercíclicos da forma $\lambda I+K$, com $K$ compacto, satisfazem o HC ou não. Vale dizer, no entanto, que o operador criado na demonstração do teorema de existência de operadores hipercíclicos (o Teorema 1.27) é, como comentamos após a demonstração do teorema, da forma $I+K$ e satisfaz o HC.

Neste capítulo temos como principal objetivo estudarmos operadores hipercíclicos da forma $\lambda I+K$. Com esse objetivo em mente, na primeira seção vamos adaptar alguns resultados envolvendo operadores hipercíclicos para operadores cíclicos uma vez que, se $T=\lambda I+S$ é hipercíclico, não é difícil ver que $S$ é cíclico. Dessa forma, fica claro como hiperciclicidade, ciclicidade e o Problema 5 se relacionam.

Lema 4.2. Se $T=\lambda I+S$ é um operador hipercíclico com vetor hipercíclico $x$, então $S$ é um operador cíclico com vetor cíclico $x$.

Entre os resultados que adaptaremos, destacamos o Critério de Ciclicidade. Como pode-se inferir pelo nome e pelo contexto, o Critério de Ciclicidade é uma adaptação do HC para operadores cíclicos. Já na segunda seção usaremos tais resultados no estudo de operadores hipercíclicos da forma $\lambda I+S$.

$\mathrm{Na}$ terceira seção vamos mostrar que o já mencionado contraexemplo de Bayart e Matheron (de um operador hipercíclico que não satisfaz o Critério de Hiperciclicidade) não pode ser adaptado para espaços com pouquíssimos operadores. Já na última seção vamos continuar no espírito de adaptar alguns resultados envolvendo hiperciclicidade, definindo assim o conceito de sub-ciclicidade. Tal conceito, como o próprio nome diz, é baseado no conceito de sub-hiperciclicidade, visto nos capítulos anteriores.

Antes de começar a primeira seção, vejamos um lema de natureza técnica que será bastante útil ao longo deste capítulo:

Lema 4.3. Seja $X$ um espaço de Banach. Seja $T, S \in \mathcal{B}(X)$ tal que $T=\lambda I+S$. Então $\mathbb{K}[T]=\mathbb{K}[S]$, onde $\mathbb{K}[x]$ denota o anel formado por todos os polinômios com coeficientes em $\mathbb{K}$

\footnotetext{
${ }^{2}$ Se $X$ é um espaço de Banach, como consequência do Teorema de Hahn-Banach para cada $x \in X$, existe um $f_{x} \in X^{*}$ tal que $f_{x}(x)=1$. Daí, usando tais funcionais e elementos quaisquer de $X$ podemos definir inúmeros operadores de posto finito em $X$ : por exemplo, se $x, y \in X$, podemos definir $T \in \mathcal{B}(X)$ por $T(z)=f_{x}(z) y+f_{y}(z) x$.
} 
e indeterminada $x$.

DEMONSTRAÇÃO:

- $\mathbb{K}[T] \subseteq \mathbb{K}[S]$

Imediato, pois $T=\lambda I+S$. Logo, todo polinômio em $T$ é, no fundo, um polinômio em $S$.

- $\mathbb{K}[T] \supseteq \mathbb{K}[S]$

Seja $p(S) \in \mathbb{K}[S]$. Mostraremos a inclusão via indução no grau do polinômio. Se o grau de $p$ é 1 , então $p(S)=a_{0} I+a_{1} S=\left(a_{0}-a_{1} \lambda\right) I+a_{1}(\lambda I+S)=\left(a_{0}-a_{1} \lambda\right) I+a_{1} T=q(T) \in \mathbb{K}[T]$, como desejado.

Suponhamos que todo polinômio em $S$ de grau menor ou igual a $n$ esteja em $\mathbb{K}[T]$. Suponha que $p(S)=a_{0} I+a_{1} S+\ldots+a_{n} S^{n}+a_{n+1} S^{n+1}$. Note que $a_{n+1} T^{n+1}=a_{n+1}(\lambda I+S)^{n+1}=$ $q(S)+a_{n+1} S^{n+1}$, com grau $q \leq n$. Portanto, $a_{n+1} S^{n+1}=a_{n+1} T^{n+1}-q(S)$. Assim:

$$
\begin{aligned}
p(S) & =a_{0} I+a_{1} S+\ldots+a_{n} S^{n}+a_{n+1} S^{n+1} \\
& =a_{0} I+a_{1} S+\ldots+a_{n} S^{n}+\left(a_{n+1} T^{n+1}-q(S)\right) \\
& =\left[a_{0} I+a_{1} S+\ldots+a_{n} S^{n}-q(S)\right]+a_{n+1} T^{n+1}
\end{aligned}
$$

Denotando $r(S):=a_{0} I+a_{1} S+\ldots+a_{n} S^{n}-q(S)$, observe que grau $r(S) \leq n$. Logo, por indução, tal polinômio pertence à $\mathbb{K}[T]$.

Como $\mathbb{K}[T]$ é um anel e $r(S), a_{n+1} T^{n+1} \in \mathbb{K}[T]$, segue então que $r(S)+a_{n+1} T^{n+1}=p(S) \in \mathbb{K}[T]$, como queríamos mostrar.

\subsection{Critério de Ciclicidade}

Como dito na introdução deste capítulo, apresentaremos agora alguns resultados de operadores hipercíclicos adaptados para operadores cíclicos. O primeiro deles é a proposição a seguir. Tal proposição é uma adaptação do Teorema da Transitividade de Birkhoff (Teorema 1.17):

Proposição 4.4. Sejam $X$ um espaço de Banach separável e de dimensão infinita e $T \in \mathcal{B}(X)$. Então $T$ é cíclico e possui um conjunto denso de vetores cíclicos se, e somente se, para quaisquer $U, V \subseteq X$ abertos não-vazios, existe um $p \in \mathbb{K}[x]$ tal que

$$
p(T)(U) \cap V \neq \emptyset
$$

DemonstraÇÃo: $(\Leftarrow)$. Denote por $C(T)$ o conjunto dos vetores cíclicos para $T$. Basta mostramos que $C(T)$ é denso: observe que, sendo $C(T)$ não-vazio, é claro que $T$ é cíclico.

Sendo $X$ separável, temos que $X$ admite uma base enumerável de abertos $\left(U_{k}\right)_{k \in \mathbb{N}}$. Assim, temos que $x \in C(T)$ se, dado $k \in \mathbb{N}$, existe $p \in \mathbb{K}[x]$ tal que $p(T) x \in U_{k}$. Ou seja, 


$$
C(T)=\bigcap_{k=1}^{\infty} \bigcup_{p \in \mathbb{K}[x]} p(T)^{-1}\left[U_{k}\right]
$$

Como $T$ é contínuo, $p(T)^{-1}\left[U_{k}\right]$ é aberto, para todo $p \in \mathbb{K}[x]$. Ainda, usando a equação em destaque no enunciado do lema, concluímos que $A_{k}:=\bigcup_{p \in \mathbb{K}[x]} p(T)^{-1}\left[U_{k}\right]$ é denso; sendo uma união de abertos, segue também que $A_{k}$ é aberto.

Pelo Teorema de Baire, temos que $\bigcap_{k=1}^{\infty} A_{k}=C(T)$ é um conjunto denso, como desejado.

$(\Rightarrow)$. Tome $U, V$ abertos não-vazios quaisquer. Como $C(T)$ é denso, então existe $x \in U \cap C(T)$. Sendo $x$ um vetor cíclico para $T$ (pois $x \in C(T)$ ), então existe um polinômio $p$ tal que $p(T) x \in V$. Ou seja, temos que $p(T)(U) \cap V \neq \emptyset$.

Observe que, diferente do Teorema da Transitividade de Birkhoff, precisamos supor que $T$ é cíclico e possui um conjunto denso de vetores cíclicos para que a equivalência seja verdade. Tal suposição faz sentido uma vez que, como veremos a seguir, nem todo operador cíclico possui um conjunto denso de vetores cíclicos (diferente de, como já vimos, dos operadores hipercíclicos).

Exemplo 4.5 (Observação $3.2 \mathrm{em}[12]$ ). Denote $U$ a bola unitária aberta em $\mathbb{C}$ (isto é, $U=$ $B(0,1) \subseteq \mathbb{C})$. Denotemos por $A^{2}(U)$ o espaço de Bergman, isto é, o subespaço de $L^{2}(U)$ formado por todas as funções holomorfas. Sendo um subespaço fechado de $L^{2}(U)$, temos que $A^{2}(U)$ é um espaço de Banach $3^{3}$

Seja $M_{z}: A^{2}(U) \rightarrow A^{2}(U)$ definido por $M_{z}(f)(z)=z f(z)$. Observe que $M_{z}$ é um operador linear bem definido e contínuo em $A^{2}(U)$. Agora, tomando a função constante igual a 1 , temos que $M_{z}(1)(z)=z$ e, portanto, $M_{z}^{n}(1)(z)=z^{n}$. Como os polinômios formam um conjunto denso em $A^{2}(U)$, não é difícil ver então que a função constante igual a 1 é um vetor cíclico para $M_{z}$. Vamos mostrar que, no entanto, $M_{z}$ não possui um conjunto denso de vetores cíclicos.

Em primeiro lugar, temos que se $K \subseteq U$ é um compacto, então vale a desigualdade

$$
\sup _{z \in K}|f(z)| \leq C_{K}\|f\|_{L^{2}(U)}
$$

onde $C_{K}$ é uma constante que depende de $K$. Dessa forma, temos que a convergência em norma implica em convergência uniforme nos conjuntos compactos $4_{4}^{4}$

Suponha agora que $f \in A^{2}(U)$ seja um vetor cíclico para $M_{z}$ tal que $f\left(z_{0}\right)=0$ para algum $z_{0} \in U$. Logo, não é difícil ver que $M_{z}^{n}(f)\left(z_{0}\right)=0$, para todo $n \in \mathbb{N}$. Seja $g \in A^{2}(U)$ uma função tal que $g\left(z_{0}\right) \neq 0$. Como $f$ é um vetor cíclico para $M_{z}$, então existe uma sequência $\left(n_{k}\right) \subseteq \mathbb{N}$ tal que $M_{z}^{n_{k}}(f) \rightarrow g$ em norma. Pela desigualdade em destaque, segue que $M_{z}^{n_{k}}(f) \rightarrow g$ compactamente, o que é um absurdo, uma vez que teríamos que $M_{z}^{n_{k}}(f)\left(z_{0}\right) \rightarrow g\left(z_{0}\right)$, o que implicaria que $0 \rightarrow g\left(z_{0}\right)$, mas $g\left(z_{0}\right) \neq 0$, por hipótese.

Dessa forma, concluímos que se $f$ é um vetor cíclico para $M_{z}$, então $f(z) \neq 0$, para todo $z \in U$. Suponha agora que o conjunto $C\left(M_{z}\right)$ dos vetores cíclicos de $M_{z}$ é denso em $A^{2}(U)$. Seja $g$ uma

\footnotetext{
${ }^{3}$ Para uma introdução detalhada ao espaço de Bergman, ver [11].

${ }^{4}$ Para uma demonstração da desigualdade, ver [11, Teorema 1, pp. 7-8].
} 
função que não é identicamente nula em $U$ e é tal que $g\left(z_{0}\right)=0$ para algum $z_{0} \in U 5$ Sendo o conjunto dos vetores cíclicos de $M_{z}$ denso em $A^{2}(U)$, então existe uma sequência $\left(f_{k}\right)_{k \in \mathbb{N}} \subseteq C\left(M_{z}\right)$ tal que $f_{k} \rightarrow g$ em norma. Como cada $f_{k}$ é um vetor cíclico, temos que $f_{k}(z) \neq 0$, para todo $z \in U$.

No entanto, usando o Teorema de Hurwitz e a Desigualdade (4.1), chegamos a uma contradição: como $f_{k}(z) \neq 0$ para todo $z \in U$ e $f_{k} \rightarrow g$, deveríamos ter então que $g(z) \neq 0$, para todo $z \in U$, uma contradição com a escolha de $g \bigsqcup^{6}$

Portanto, segue que $M_{z}$ não possui um conjunto denso de vetores cíclicos.

Sabendo que o Teorema da Transitividade de Birkhoff é usado na demonstração do Critério de Hiperciclicidade (vide a demonstração do HC no Capítulo 1) e continuando no espírito de adaptarmos resultados conhecidos de hiperciclicidade, obtemos um "Critério de Ciclicidade".

Como veremos a seguir, tal critério é uma adaptação exata do HC. Esta adaptação foi realizada por Grivaux, em [13], embora tenhamos feita a mesma adaptação independentemente, sem o conhecimento da existência de tal resultado no artigo mencionado. É importante ressaltar que Grivaux enuncia as mesmas hipóteses, mas acaba demonstrando que $T \oplus T$ é cíclico (o que evidentemente mostra que $T$ é cíclico) 7 Uma vez que nossa tese é diferente (concluímos apenas que $T$ é cíclico), apresentamos uma demonstração obtida independentemente daquela feita por Grivaux que, como é de se imaginar, é baseada na proposição anterior e na demonstração do Critério de Hiperciclicidade.

Teorema 4.6 (Critério de Ciclicidade, [13]). Sejam X um espaço de Banach separável de dimensão infinita e $T: X \rightarrow X$ um operador linear limitado. Suponha que existem subconjuntos $X_{0}, Y_{0}$ densos em $X$, uma sequência de polinômios $\left(p_{n}\right)_{n \in \mathbb{N}} \subseteq \mathbb{K}[x]$ e funções $S_{n}: Y_{0} \rightarrow X$ tais que:

(i) $p_{n}(T)(x) \rightarrow 0$, para todo $x \in X_{0}$.

(ii) $S_{n}(y) \rightarrow 0$, para todo $y \in Y_{0}$.

(iii) $p_{n}(T) \circ S_{n}(y) \rightarrow y$, para todo $y \in Y_{0}$.

Então T é cíclico.

Demonstração: A demonstração é completamente análoga àquela feita no primeiro capítulo para o HC. De fato, sejam $U, V$ dois abertos não-vazios de $X$. Pela densidade de $X_{0}$ e $Y_{0}$, podemos achar $x, y$ tais que $x \in X_{0} \cap U$ e $y \in Y_{0} \cap V$. Como $x \in X_{0}$, então $p_{n}(T)(x) \rightarrow 0$, pela condição (i) da hipótese. Da mesma maneira, da condição (ii) temos que $S_{n}(y) \rightarrow 0$. Logo, definindo $x_{n}:=x+S_{n}(y)$, temos que $x_{n} \rightarrow x$. Portanto, como $x \in U$, existe um $k_{1} \in \mathbb{N}$ tal que, para todo $k>k_{1}$, temos que $x_{k} \in U$.

\footnotetext{
${ }^{5} \mathrm{Um}$ exemplo de função $g$ seria $g(z)=z$.

${ }^{6} \mathrm{O}$ enunciado do Teorema de Hurwitz é o seguinte: "Se as funções $\left(f_{n}\right)_{n \in \mathbb{N}}$ são analíticas e não se anulam numa região $\Omega$ e a sequência $\left(f_{n}\right)_{n \in \mathbb{N}}$ converge para uma função $f$ (seja uniformemente ou em cada subconjunto compacto de $\Omega$ ), então $f$ ou é identicamente nula ou não se anula em $\Omega$ ”. Tal enunciado foi retirado de [1, Teorema 2, Capítulo 5, Seção 1, p. 178].

${ }^{7}$ Vamos apresentar na Proposição 4.10 exatamente este resultado: se $T$ satisfaz o Critério de Ciclicidade, então $T \oplus T$ é cíclico.
} 
Sendo $T$ um operador linear, temos que

$$
p_{n}(T)\left(x_{n}\right)=p_{n}(T)\left(x+S_{n}(y)\right)=p_{n}(T)(x)+p_{n}(T) \circ S_{n}(y) \rightarrow y
$$

usando as condições (i) e (iii) da hipótese. Logo, como $y \in V$, existe um $k_{2} \in \mathbb{N}$ tal que, para todo $k>k_{2}$, devemos ter $p_{k}(T)\left(x_{k}\right) \in V$.

Dessa forma, tomando um $k_{0} \geq \max \left\{k_{1}, k_{2}\right\}$, temos que, para todo $k>k_{0}$, ambos $x_{k} \in U$ e $p_{k}(T)\left(x_{k}\right) \in V$ são satisfeitos. Portanto, segue que $p_{k}(T)(U) \cap V \neq \emptyset$, para todo $k>k_{0}$. Logo, pela Proposição 4.4. T é cíclico, como desejado.

Observação 4.7. Assim como fizemos na Observação 1.15 para o HC, podemos reescrever as condições (ii) e (iii) do Critério de Ciclicidade da seguinte maneira equivalente: "dado $y \in Y_{0}$, existe uma sequência $\left(x_{n}\right)_{n \in \mathbb{N}} \subseteq X$ tal que $x_{n} \rightarrow 0$ e $p_{n}(T) x_{n} \rightarrow y "$.

Assim como no caso do Critério de Hiperciclicidade, quando necessário faremos referência ao Critério de Ciclicidade pela sigla CC 8 Observe que assim como no caso do $\mathrm{HC}$, as funções $S_{n}$ exigidas pelo CC não precisam ser lineares nem contínuas.

Uma vez que usamos a Proposição 4.4 na demonstração do Critério de Ciclicidade, fica provado o seguinte corolário:

Corolário 4.8. Se T satisfaz o Critério de Ciclicidade, então $T$ possui um conjunto $G_{\delta}$ denso de vetores cíclicos.

Com este corolário, o Exemplo 4.5 fornece um exemplo de um operador cíclico que não satisfaz o Critério de Ciclicidade. Por fim, temos mais um corolário envolvendo o Critério de Ciclicidade:

Corolário 4.9. Sejam $T, S$ operadores tais que $T=\lambda I+S$. Suponha que $T$ ou $S$ satisfaçam o Critério de Ciclicidade. Então ambos satisfazem o Critério de Ciclicidade.

DemonstraÇão: Note que basta provarmos o caso em que $T$ satisfaz o CC, uma vez que $S=$ $(-\lambda) I+T$. Neste caso, sabemos que existem conjuntos densos $X_{0}, Y_{0}$, polinômios $p_{n}$ e funções $S_{n}$ que satisfazem (i), (ii) e (iii) para $T$. Pelo Lema 4.3, temos que para cada $n \in \mathbb{N}$ existe $q_{n} \in \mathbb{K}[x]$ tal que $p_{n}(T)=q_{n}(S)$. Logo, usando essa igualdade e os mesmos conjuntos densos $X_{0}, Y_{0}$ e funções $S_{n}$, segue que $S$ satisfaz (i), (ii) e (iii) do CC.

Lembramos agora do Teorema 1.20 nele, mostramos que um operador hipercíclico satisfaz o HC se, e somente se, $T \oplus T$ for hipercíclico. Tendo em vista o Critério de Ciclicidade, é natural imaginar se um resultado análogo vale para operadores cíclicos. Na proposição a seguir, mostramos que uma das implicações é válida. Mais uma vez, a demonstração da proposição é adaptada do resultado análogo envolvendo hiperciclicidade e é bastante similar à demonstração que Grivaux fez em [13] para o Critério de Ciclicidade (como mencionamos anteriormente):

\footnotetext{
${ }^{8}$ Do inglês, Cyclicity Criterion.
} 
Proposição 4.10 (Grivaux, [13]). Se T satisfaz o Critério de Ciclicidade, então T $\oplus$ T é cíclico.

DemonstraçÃo: Mostremos que, dados $U=U_{1} \times U_{2}$ e $V=V_{1} \times V_{2}$ abertos, existe $p \in \mathbb{K}[x]$ tal que $p(T \oplus T)(U) \cap V \neq \emptyset$. Daí, pela Proposição 4.4, teremos o desejado.

Seja $x_{j} \in U_{j} \cap X_{0}$ e $y_{j} \in V_{j} \cap Y_{0}$, para $j=1,2$, onde $X_{0}$ e $Y_{0}$ são os conjuntos densos descritos no CC (e, por serem densos, garantem a existência de $x_{j}, y_{j}$ ).

Usando a sequência de polinômios $\left(p_{n}\right)_{n \in \mathbb{N}}$ descrita no CC, temos que

$$
p_{n}(T)\left(x_{j}+S_{n} y_{j}\right)=p_{n}(T) x_{j}+p_{n}(T) \circ S_{n}\left(y_{j}\right)
$$

para $j=1,2$.

Para $j=1$, note agora que $x_{1}+S_{n} y_{1} \rightarrow x_{1} \in U_{1}$ (vide (ii) do CC, uma vez que $y_{1} \in Y_{0}$ ) e $p_{n}(T) x_{1}+p_{n}(T) \circ S_{n}\left(y_{1}\right) \rightarrow y_{1} \in V_{1}$ (vide (i) e (iii) do CC, uma vez que $x_{1} \in X_{0}$ e $y_{1} \in Y_{0}$ ).

Portanto, podemos tomar um $k_{1} \in \mathbb{N}$ tal que, para todo $n \geq k_{1}, x_{1}+S_{n} y_{1} \in U_{1}$ e $p_{n}(T) x_{1}+$ $p_{n}(T) \circ S_{n}\left(y_{1}\right) \in V_{1}$. Analogamente, existe um $k_{2} \in \mathbb{N}$ tal que, para todo $n \geq k_{2}, x_{2}+S_{n} y_{2} \in U_{2}$ e $p_{n}(T) x_{2}+p_{n}(T) \circ S_{n}\left(y_{2}\right) \in V_{2}$.

Tome $k_{0} \geq \max \left(k_{1}, k_{2}\right)$. Denote $x_{n}=x_{1}+S_{n} y_{1}$ e $y_{n}=x_{2}+S_{n} y_{2}$, para todo $n \in \mathbb{N}$. Assim, do parágrafo anterior segue que, para todo $n \geq k_{0}$ :

$$
\left\{\begin{array}{l}
x_{n} \in U_{1} \\
p_{n}(T) x_{n} \in V_{1} \\
y_{n} \in U_{2} \\
p_{n}(T) y_{n} \in V_{2}
\end{array}\right.
$$

Portanto, para $n \geq k_{0}$, temos que $\left(x_{n}, y_{n}\right) \in U=U_{1} \times U_{2}$ e $p_{n}(T \oplus T)\left(x_{n}, y_{n}\right) \in V=V_{1} \times V_{2}$. Ou seja, tomando $p=p_{m}$, onde $m \geq k_{0}$, vale que $p(T \oplus T)(U) \cap V \neq \emptyset$, como queríamos demonstrar.

Observe também que podemos usar o lema anterior para mostrar que todo operador que satisfaz o CC é cíclico. De fato, o lema acima nos diz que se $T$ satisfaz o Critério da Ciclidade, então $T \oplus T$ é cíclico. Ora, se $T \oplus T$ é cíclico, então $T$ é cíclico, como desejado.

Antes de prosseguir e discutir se a outra implicação é válida ou não, usaremos este último lema para responder uma pergunta importante: será que todo operador cíclico satisfaz o Critério de Ciclicidade? Vimos, como consequência do Exemplo 4.5 e do Corolário 4.8, que a resposta é negativa e que, ao contrário do que aconteceu com a pergunta análoga no caso de operadores hipercíclicos, tal resposta é bastante simples quando comparada à construção de Bayart e Matheron. Agora, apenas para ilustrar a utilidade da proposição acima, mostraremos um outro exemplo que também responde tal pergunta.

Exemplo 4.11 (Halmos, [15, p.87]). Seja $X=\ell_{2}$ e considere o forward shift $F$ em $\ell_{2}$; isto é, se $\left(e_{i}\right)_{i=1}^{\infty}$ é a base canônica de $\ell_{2}$, então $F\left(e_{i}\right)=e_{i+1}$.

É claro que $F$ é um operador cíclico (um de seus vetores cíclicos é $e_{1}$ ). Vamos mostrar que $F$ não satisfaz o Critério de Ciclicidade. Para isso, faremos uso da Proposição 4.10 mostraremos que $F \oplus F$ não é cíclico. 
Suponha que $F \oplus F$ é cíclico em $\ell_{2} \oplus \ell_{2}$. Se $\langle\cdot, \cdot\rangle$ é o produto interno usual em $\ell_{2}$, vamos denotar por $\langle\cdot, \cdot\rangle_{2}$ o produto interno em $\ell_{2} \oplus \ell_{2}$ dado por $\langle(a, b),(u, v)\rangle_{2}:=\langle a, u\rangle+\langle b, v\rangle$. Observe que a norma gerada pelo produto interno $\langle\cdot, \cdot\rangle_{2}$ é a mesma que é normalmente utilizada em somas diretas externas, a saber, $\|(x, y)\|_{X \oplus X}=\left(\|x\|_{X}^{2}+\|y\|_{X}^{2}\right)^{1 / 2}$ (vide a Definição 1.9.

Tome $x=\left(x_{i}\right)_{i=1}^{\infty}$ e $y=\left(y_{i}\right)_{i=1}^{\infty}$ tal que $(x, y)$ é um vetor cíclico para $F \oplus F$. Seja $\left(\alpha_{1}, \beta_{1}\right) \in \mathbb{K}^{2}$ tal que $\left(\alpha_{1}, \beta_{1}\right)$ é ortogonal a $\left(x_{1}, y_{1}\right)$ e $\alpha_{1}, \beta_{1} \neq 0$.

Considere agora os vetores $\alpha=\left(\alpha_{i}\right)_{i=1}^{\infty}$ e $\beta=\left(\beta_{i}\right)_{i=1}^{\infty}$, onde $\alpha_{1}, \beta_{1}$ são aqueles encontrados no parágrafo anterior e $\alpha_{i}=\beta_{i}=0$, para todo $i \geq 2$. Observe que $\|(\alpha, \beta)\|_{\ell_{2} \oplus \ell_{2}} \neq 0$. Dessa forma, não é difícil ver que $(\alpha, \beta)$ é ortogonal ao conjunto span orb $((x, y), F \oplus F)$, o que contradiz o fato de $F \oplus F$ ser cíclico 9

Observe que o operador $S$ no exemplo acima é claramente não-hipercíclico, uma vez que $\|S\|=1$ e todo operador hipercíclico $T$ é tal que $\|T\|>1$. Veremos ainda nesta seção um exemplo de um operador hipercíclico que não satisfaz o Critério de Ciclicidade 10

Vamos, agora, ver se a outra implicação vale ou não. Comecemos com o seguinte lema:

Lema 4.12 (Observação $5.1 \mathrm{em}[13]$ ). Se $T \oplus T$ é cíclico, então $\sigma_{p}\left(T^{*}\right)=\emptyset$.

DemonstraÇão: Suponha que $\sigma_{p}\left(T^{*}\right) \neq \emptyset$. Seja $\alpha \in \sigma_{p}\left(T^{*}\right)$. Logo, pelo Lema 1.2 , temos que $T-\bar{\alpha} I$ não tem imagem densa. Dessa forma, a imagem de $T-\bar{\alpha} I \oplus T-\bar{\alpha} I$ tem codimensão ao menos 2, o que mostra que $T-\bar{\alpha} I \oplus T-\bar{\alpha} I$ não é cíclico (uma vez que, se $S$ é cíclico, então a codimensão da imagem de $S$ é no máximo 1$)$.

Agora, se $S=T-\bar{\alpha} I$, então pelo Lema 4.3 temos que $\mathbb{K}[S]=\mathbb{K}[T]$, o que mostra que $T \oplus T$ também não é cíclico, como desejado.

Observe que não é verdade que se $T$ é cíclico então $\sigma_{p}\left(T^{*}\right)=\emptyset$ uma vez que, em geral, um operador cíclico não necessariamente tem imagem densa (e, portanto, temos que $0 \in \sigma_{p}\left(T^{*}\right)$ ). De fato, seja $F$ o forward shift como no Exemplo 4.11. Como mencionado no exemplo, temos que $e_{1}$ é um vetor cíclico para $F$. No entanto, $\operatorname{Im} F=\overline{\operatorname{span}\left\{e_{n}: n \geq 2\right\}}$ e daí não é difícil ver que $e_{1} \notin \operatorname{Im} F$, o que mostra que $\operatorname{Im} F$ não tem imagem densa 11

Vamos agora demonstrar a volta da Proposição 4.10. Ao contrário do que acontece com hiperciclicidade, a volta não é "imediata": embora a demonstração da volta seja análoga à demonstração de que " $T \oplus T$ é hipercíclico $\Rightarrow T$ satisfaz o $H C$ ", temos que colocar uma hipótese adicional para que o resultado seja verdade. Discutiremos a necessidade de tal hipótese adicional após a demonstração do resultado.

\footnotetext{
${ }^{9}$ De fato, se existisse uma sequência $\left(a_{n}, b_{n}\right) \in \operatorname{spanorb}((x, y), F \oplus F)$ tal que $\left(a_{n}, b_{n}\right) \rightarrow(\alpha, \beta)$, tomando o funcional $f((z, w))=\langle(z, w),(\alpha, \beta)\rangle_{2}$, teríamos que $f\left(a_{n}, b_{n}\right) \rightarrow f(\alpha, \beta) \Rightarrow 0 \rightarrow\langle(\alpha, \beta),(\alpha, \beta)\rangle=\|(\alpha, \beta)\|_{\ell_{2} \oplus \ell_{2}}^{2} \neq 0$, uma contradição.

${ }^{10}$ Observe que tal exemplo será fundamentalmente diferente do Exemplo 4.5 uma vez que todo operador hipercíclico possui um conjunto $G_{\delta}$ de vetores hipercíclicos (e, portanto, cíclicos), independentemente de satisfazer ou não o HC (vide o Teorema 1.17). Logo, não conseguimos utilizar o Corolário 4.8 para mostrar que um operador hipercíclico não satisfaz o CC.

${ }^{11}$ Apenas por curiosidade, se $T$ for hipercíclico, então temos que $\sigma_{p}\left(T^{*}\right)=\emptyset$; uma referência para este resultado é [14, Lema 2.53, pp.52-53].
} 
Proposição 4.13. Seja X um espaço de Banach complexo. Se $T \oplus T$ é cíclico, então $T$ satisfaz o Critério de Ciclicidade.

DemonstraÇÃo: Seja $(x, y)$ um vetor cíclico para $T \oplus T$. É claro que $x$ e $y$ são vetores cíclicos para $T$. Seja $p \in \mathbb{C}[X]$ um polinômio não-nulo. Vamos mostrar que $p(T) y$ é um vetor cíclico para $T$. Como $X$ é um espaço complexo e $\sigma_{p}\left(T^{*}\right)=\emptyset$ (vide o lema anterior), então usando o Lema 1.2 não é difícil ver que $p(T)$ tem imagem densa, uma vez que as operações de composição e multiplicação por um escalar não-nulo preservam a densidade da imagem. Logo, dado um aberto $V$ qualquer, temos que $[p(T)]^{-1}(V)$ é um aberto não-vazio. Sendo $y$ um vetor cíclico, então existe $q \in \mathbb{C}[X]$ tal que $q(T) y \in[p(T)]^{-1}(V)$. Portanto, temos que $p(T) q(T) y=q(T)[p(T) y] \in V$, o que mostra que $p(T) y$ é um vetor cíclico para $T$.

Afirmamos agora que $(x, p(T) y)$ é um vetor cíclico para $T \oplus T$. De fato, como vimos no parágrafo anterior, temos que $p(T)$ tem imagem densa. Logo, se $I$ é o operador identidade em $X$, então $I \oplus p(T)$ tem imagem densa. Agora, como $D=\{(q(T) x, q(T) y): q \in \mathbb{C}[X]\}$ é denso em $X \oplus X$ (pois $T \oplus T$ é cíclico), então $(I \oplus p(T))(D)=\{(q(T) x, p(T) q(T) y): q \in \mathbb{C}[X]\}=\{(q(T) x, q(T) p(T) y): q \in$ $\mathbb{C}[X]\}=\operatorname{span} \operatorname{orb}((x, p(T) y), T \oplus T)$ também é denso em $X \oplus X$, o que mostra que $(x, p(T) y)$ é um vetor cíclico para $T \oplus T{ }^{12}$

Uma vez que, dado um aberto não-vazio $U \subseteq X$, existe um $p \in \mathbb{C}[X]$ tal que $p(T) y \in U$, considerando os dois parágrafos anteriores podemos dizer que para todo aberto não-vazio $U$ existe um $u \in U$ tal que $(x, u)$ é um vetor cíclico para $T \oplus T$.

Denote por $B_{k}=B(0,1 / k)$. Pelo que já comentamos, fixado $k \in \mathbb{N}$, podemos achar $u_{k} \in B_{k}$ tal que $\left(x, u_{k}\right)$ é um vetor cíclico para $T \oplus T$. Logo, por indução, podemos encontrar uma sequência $\left(p_{k}\right)_{k \in \mathbb{N}} \subseteq \mathbb{C}[X]$ de polinômios tais que

$$
\begin{array}{r}
p_{k}(T)(x) \in B_{k} \\
p_{k}(T)\left(u_{k}\right) \in x+B_{k}
\end{array}
$$

Seja $X_{0}=Y_{0}:=\operatorname{spanorb}(x, T)$. Sendo $x$ um vetor cíclico, $X_{0}, Y_{0}$ são densos em $X$ e, portanto, são candidatos a serem os conjuntos exigidos no Critério de Ciclidade. Por 4.2, temos que $p_{k}(T)(x) \rightarrow 0$. Daí, se $z \in X_{0}=\operatorname{spanorb}(x, T)$, então $z=q(T) x$ e, portanto, $p_{k}(T)(z)=p_{k}(T)(q(T) x)=q(T)\left(p_{k}(T) x\right) \rightarrow q(T) 0=0$, satisfazendo assim a primeira condição do Critério de Ciclicidade.

Definimos agora $S_{k}: Y_{0} \rightarrow X$ da seguinte maneira: $S_{k}(q(T) x):=q(T) u_{k}$. Como $u_{k} \in B_{k}$, então $u_{k} \rightarrow 0$. Logo, se $z \in Y_{0}, S_{k}(z)=S_{k}(q(T) x)=q(T) u_{k} \rightarrow 0$, satisfazendo então a segunda condição.

Verifiquemos agora a terceira condição. Seja $z=q(T) x \in Y_{0}$. Logo, temos que $p_{k}(T) \circ S_{k}(z)=$ $p_{k}(T) \circ S_{k}(q(T) x)=p_{k}(T)\left(q(T) u_{k}\right)=q(T)\left(p_{k}(T) u_{k}\right) \rightarrow q(T) x=z$, vide a Equação 4.3). Logo, a terceira condição do Critério de Ciclicidade está satisfeita.

Satisfeitas as três condições do Critério de Ciclicidade, fica provada a tese.

\footnotetext{
${ }^{12}$ Note que, a rigor, $(I \oplus p(T))(D)$ é denso na imagem de $I \oplus p(T)$. No entanto, como a imagem de $I \oplus p(T)$ é densa em $X \oplus X$, então segue que $(I \oplus p(T))(D)$ é denso em $X \oplus X$.
} 
Note que as funções $S_{k}$ estão bem definidas mas podem não ser lineares nem contínuas. Porém, como dito anteriormente, isto não cria um problema com o Critério de Ciclicidade e não torna esta demonstração inválida.

Observe que usamos o fato de que $X$ era um espaço complexo para garantir que $p(T)$ tem imagem densa; se $X$ fosse um espaço real, não temos essa garantia, uma vez que a hipótese de que $\sigma_{p}\left(T^{*}\right)=\emptyset$ (obtido através do Lema 4.12) não é suficiente para mostrar que, se $p$ é um polinômio irredutível de grau 2 sobre $\mathbb{R}$, então $p(T)$ tem imagem densa. Tal situação não acontece com operadores hipercíclicos, como vimos no Teorema 1.31 .

Portanto, se supusermos que $T$ é hipercíclico, ao analisar a demonstração da Proposição 4.13 , podemos dispensar a hipótese de que $X$ é um espaço de Banach complexo. Logo, para operadores hipercíclicos, a equivalência entre $T \oplus T$ ser cíclico e $T$ satisfazer o CC vale. Tal observação é importante e nos leva a um "aprimoramento" do Teorema 1.20

Teorema 4.14. Seja T um operador hipercíclico em um espaço de Banach. São equivalentes:

(i) T satisfaz o Critério de Hiperciclicidade.

(ii) $T \oplus T$ é hipercíclico.

(iii) $T \oplus T$ é cíclico.

(iv) T satisfaz o Critério de Ciclicidade.

Demonstração: Pelo Teorema 1.20, temos que $T$ satisfaz o $\mathrm{HC}$ se, e somente se, $T \oplus T$ é cíclico se, e somente se, $T \oplus T$ é hipercíclico. Pela Proposição 4.10, temos que se $T$ satisfaz o CC, então $T \oplus T$ é cíclico. Ainda, se $T$ satisfaz o HC, é claro que $T$ satisfaz o CC.

Com este teorema em mãos, podemos demonstrar que um operador hipercíclico satisfaz o HC usando o CC. A vantagem dessa abordagem é que podemos usar uma sequência de polinômios em $T$ ao invés de potências de $T$ : teoricamente, como toda potência de $T$ é um polinômio em $T$, então é mais fácil de mostrar que um operador hipercíclico satisfaz o CC do que o $\mathrm{HC}$.

Outra consequência do teorema anterior é que rapidamente obtemos outro exemplo de um operador cíclico que não satisfaz o Critério de Ciclicidade: o operador hipercíclico que não satisfaz o HC construído por Bayart e Matheron.

\subsection{Operadores Hipercíclicos da Forma $\lambda I+S$}

Vamos agora utilizar os resultados da seção anterior para o estudo de operadores da forma $\lambda I+S$. Como já dissemos, um fator motivante para o estudo das propriedades de operadores desta forma vêm dos espaços com pouquíssimos operadores (isto é, espaços onde todo operador linear é da forma $\lambda I+K$, com $K$ compacto). Porém, os resultados desta seção não farão uso da compacidade. Por isso, optamos por escrever $\lambda I+S$ ao invés de $\lambda I+K$ para deixar claro que não estamos, a priori, supondo que $S$ é compacto. 
Dessa maneira, os resultados que veremos a seguir também podem ser usados em espaços separáveis onde seus operadores lineares também são perturbações da identidade 13 Um exemplo de espaço com esta condição é o famoso espaço de Gowers-Maurey $X_{G M}$, onde todo operador linear é da forma $\lambda I+S$, com $S$ sendo um operador estritamente singular.14

O primeiro resultado é um simples corolário:

Corolário 4.15. Se $T=\lambda I+S$ é hipercíclico, então todo vetor hipercíclico de $T$ é um vetor cíclico para $S$. Além disso, $S$ possui um conjunto $G_{\delta}$ denso de vetores cíclicos.

Demonstração: A primeira parte é imediata do Lema 4.2. A segunda parte segue imediatamente da primeira, uma vez lembrado que todo operador hipercíclico possui um conjunto $G_{\delta}$ denso de vetores hipercíclicos, como visto no Teorema 1.17.

Como já discutimos anteriormente, em geral um operador cíclico não tem imagem densa. Agora, se $\lambda I+S$ for hipercíclico, então temos a garantia de que $S$ tem imagem densa, como mostra o lema a seguir:

Lema 4.16. Se $T=\lambda I+S$ é hipercíclico, então $S$ tem imagem densa. Além disso, se $p \in \mathbb{K}[x]$ é um polinômio não-nulo, então $p(S)$ tem imagem densa.

DemonstraÇÃo: Observe que $S=T-\lambda I=p(T)$, onde $p(x)=x-\lambda$. Pelo Teorema 1.31, temos que $p(T)=S$ tem imagem densa, como desejado.

A segunda parte é análoga: se $p \in \mathbb{K}[x]$ é um polinômio não-nulo, então pelo Lema 4.3 existe um polinômio não-nulo $q \in \mathbb{K}[x]$ tal que $p(S)=q(T)$. Logo, como $q(T)$ tem imagem densa (vide o Teorema 1.31), temos que $p(S)$ tem imagem densa, como desejado.

No caso específico de $T=\lambda I+S$, obtemos uma versão modificada do Teorema 4.14, que leva em conta a ciclicidade do operador $S$ e o Critério de Ciclicidade:

Teorema 4.17. Sejam $X$ um espaço de Banach e $T=\lambda I+S$ um operador hipercíclico. São equivalentes:

(i) T satisfaz o Critério de Hiperciclicidade.

(ii) S satisfaz o Critério de Ciclicidade.

(iii) $S \oplus S$ é cíclico.

(iv) $T \oplus T$ é cíclico.

(v) $T \oplus T$ é hipercíclico.

\footnotetext{
${ }^{13} \mathrm{Ou}$ seja, são da forma $\lambda I+S$.

${ }^{14}$ Sejam $X, Y$ espaços de Banach. Um operador linear limitado $T: X \rightarrow Y$ é estritamente singular quando não existe subspaço $E \subseteq X$ tal que $\left.T\right|_{E}: E \rightarrow T(E)$ é um isomorfismo (ou seja, $\left.T\right|_{E}$ é um isomorfismo sobre a sua imagem).
} 
DemonstraÇÃo: (i) $\Rightarrow$ (ii). Seja $\left(n_{j}\right)$ a sequência crescente de inteiros positivos fornecida pelo HC. Seja $p_{j}(x)=(\lambda+x)^{n_{j}}$. Claro que $p_{j} \in \mathbb{K}[x]$. Ainda, observe que

$$
p_{j}(S)=(\lambda I+S)^{n_{j}}=T^{n_{j}}
$$

Para provarmos o CC, tome os conjuntos densos $X_{0}$ e $Y_{0}$ fornecidos pelo HC. Como $T^{n_{j}}(x) \rightarrow 0$, para todo $x \in X_{0}$, temos, pela última equação em destaque, que $p_{j}(S)(x) \rightarrow 0$, para todo $x \in X_{0}$. Isto prova (i) do CC.

Para provarmos (ii) e (iii) do CC, tomemos as funções $S_{j}$ como sendo as funções $S_{n_{j}}$ fornecidas pelo HC ${ }^{15}$ Assim, pelo $\mathrm{HC}$, temos que $S_{n_{j}}(y) \rightarrow 0$, para todo $y \in Y_{0}$. Portanto, $S_{j}(y) \rightarrow 0$, para todo $y \in Y_{0}$, provando (ii) do CC.

Para provar (iii), note que, dado $y \in Y_{0}, p_{j}(S) \circ S_{j}(y)=T^{n_{j}} \circ S_{n_{j}}(y) \rightarrow y$, vide o (iii) do HC e a equação anterior em destaque.

Portanto, $S$ satisfaz (i), (ii) e (iii) do CC, como desejado.

(ii) $\Rightarrow$ (iii). Proposição 4.10 .

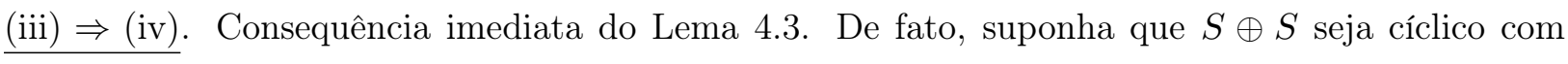
vetor cíclico $(x, y)$. Logo, dados $(U, V)$ abertos quaisquer, podemos achar $p$ polinômio tal que $p(S) x \in U$ e $p(S) y \in V$. Pelo Lema 4.3, existe um $q$ polinômio tal que $q(T)=p(S)$. Logo, temos que $q(T) x \in U$ e $q(T) y \in V$, o que mostra que $T \oplus T$ é cíclico, com vetor cíclico $(x, y)$.

(iv) $\Rightarrow(\mathrm{v})$ e (v) $\Rightarrow(\mathrm{i})$. Teorema 1.20 .

Pelo Corolário 1.28 sabemos que, dado um espaço de Banach separável $X$, existe um operador $T=I+K \in B(X)$, com $K$ compacto, que satisfaz o HC. Portanto, pelo teorema anterior segue que $K$ satisfaz o CC. Assim, temos que existem operadores que satisfazem o CC (isto é, o CC não é uma condição suficiente que é impossível de ser alcançada; logo o resultado é "válido"). Observe ainda que, sendo $K$ compacto, então ele não é hipercíclico (ver [14, Teorema 5.11, p.142]) e, portanto, não pode satisfazer o HC. Logo, $K$ satisfaz o CC mas não o HC, mostrando que a hipótese do Teorema 4.14 é realmente necessária 16

Corolário 4.18. Sejam $T, S$ operadores hipercíclicos tais que $T=\lambda I+S$. Suponha que $T$ ou $S$ satisfaçam o Critério de Hiperciclicidade. Então ambos satisfazem o Critério de Hiperciclicidade.

DemonstraÇÃo: Assim como no Corolário 4.9, basta provarmos o caso de $T$ satisfazer o HC, já que $S=(-\lambda) I+T$. Neste caso, pelo Teorema 4.17, temos que $S$ satisfaz o CC. Sendo $S$ hipercíclico, pelo Teorema 4.14 segue que $S$ satisfaz o HC, como desejado.

\footnotetext{
${ }^{15}$ Vale frisar que as funções $S_{j}$ tem nenhuma relação com $S=T-\lambda I$.

${ }^{16}$ Isto é, se $T$ não é hipercíclico, então a equivalência " $T$ satisfaz o HC se, e somente se, $T$ satisfaz o $C C$ " não necessariamente é verdade.
} 
Exemplo 4.19. Seja $X=c_{0}$ ou $X=\ell_{p}$, para $1 \leq p<\infty$. Seja $w \in \mathbb{K}$ tal que $|w|>1$. Vimos, no Exemplo 1.18, que o backward shift $B_{w}$ definido por $B_{w}\left(x_{1}, x_{2}, x_{3}, \ldots\right)=w \cdot\left(x_{2}, x_{3}, x_{4}, \ldots\right)$ é hipercíclico e satisfaz o HC. Logo, pelo corolário anterior, se $I+B_{w}$ for hipercíclico, então $I+B_{w}$ também satisfaz o HC.

Como vimos no Exemplo 1.22 não só $I+B_{w}$ é hipercíclico (e, portanto, satisfaz o HC), ele também é mixing.

No contexto dos espaços com pouquíssimos operadores, temos que o Critério de Ciclicidade junto com o Teorema 4.17- pode vir a ser útil para determinar se um operador hipercíclico satisfaz ou o HC. Ao invés de estudarmos o operador $T=I+K$ (que, sendo uma soma de operadores, torna o estudo de orb $(x, T)$ e outras propriedades mais difícil) podemos estudar apenas o operador $K$ que, de certa maneira, é a parte mais interessante de $I+K=T$. Se mostrarmos que $K$ satisfaz o CC ou que $K \oplus K$ é cíclico então temos, justamente pelo Teorema 4.17, que $T$ satisfaz o HC.

\subsubsection{O Caso em que $S$ é Compacto}

Lema 4.20 (Lema 5.19 de [14]). Seja $X$ um espaço de Banach. Se $T=\lambda I+K$, onde $\lambda \neq 0$ e $K$ é compacto, é hipercíclico então $|\lambda|=1$ e $K$ é quasi-nilpotente (isto é, $\lim \left\|K^{n}\right\|^{1 / n}=0$ ).

Segundo esse lema, se estivermos sobre um espaço real podemos considerar apenas os operadores da forma $I+K$ (visto que se $T$ é hipercíclico então $-T$ também o é, vide a Proposição 1.30). Além disso, segundo o Corolário 1.13, todo operador hipercíclico da forma $I+K$ é invertível.

O que nos motivou neste capítulo a estudar a relação entre ciclicidade e hiperciclicidade foi o Problema 5 . desde o começo, estamos apresentando resultados envolvendo operadores da forma $\lambda I+S$ com a intenção de usar o espaço de Argyros-Haydon para dar uma resposta positiva a este problema. No entanto, não fomos capazes de usar tais ferramentas para obter um resultado significativo nesta direção.

Somos levados, então, a tentar responder tal problema negativamente. Uma das primeiras ideias é tentar reproduzir o contraexemplo de Bayart e Matheron (vide o Teorema 1.19) no espaço $X_{A H}$. Este é o assunto da próxima seção.

\subsection{O Contraexemplo de Bayart e Matheron no Espaço de Argyros-Haydon}

Apesar de não termos citado anteriormente, o contraexemplo de Bayart e Matheron é baseado em hipóteses um pouco mais gerais do que aquelas feitas no Teorema 1.19 , como veremos abaixo:

Teorema 4.21 (Bayart e Matheron, [7]). Seja X um espaço de Banach. Suponha que X admita uma base incondicional normalizada $\left(e_{i}\right)_{i=0}^{\infty}$ tal que o seu forward shift associado seja continuo ${ }^{17}$ Então existe um operador hipercíclico $T \in \mathcal{B}(X)$ tal que $T \oplus T$ não é hipercíclico (e, portanto, $T$ não satisfaz o Critério de Hiperciclicidade) ${ }^{18}$

\footnotetext{
${ }^{17}$ Isto é, se $\left(e_{i}\right)_{i=0}^{\infty}$ é uma base e $E=\operatorname{span}\left\{e_{i}: i=0,1,2 \ldots\right\}=c_{00}$, então o forward shift associado à base $\left(e_{i}\right)_{i=0}^{\infty}$ é o operador linear $S: E \rightarrow E$ dado por $S\left(e_{i}\right)=e_{i+1}$.

${ }^{18}$ Vide o Teorema 1.20
} 
Não é difícil ver que os espaços $c_{0}$ e $\ell_{p}(\operatorname{com} 1 \leq p<\infty)$ satisfazem as hipóteses do teorema acima e, portanto, o Teorema 1.19 é de fato um corolário do teorema anterior.

Ao analisar tal teorema procurando adaptá-lo para o espaço de Argyros-Haydon, imediatamente surge um grande problema: a hipótese do espaço de Banach $X$ admitir uma base incondicional. Sendo o espaço de Argyros-Haydon $X_{A H}$ um espaço com pouquíssimos operadores, então $X_{A H}$ não possui uma base incondicional - apesar de possuir uma base de Schauder.

De fato, suponhamos que $X_{A H}$ tenha base incondicional. Então claramente $X_{A H}$ é decomponível. Logo, se $P$ é uma projeção em $X_{A H}$ tal que $X=\operatorname{ker} P \oplus \operatorname{Im} P$, então $P=\lambda I+K$. Com isso, do fato de que devemos ter $P^{2}=P$ (pois $P$ é projeção) obtemos que $\lambda=0$ ou $\lambda=1$. Como $P$ é uma projeção se, e somente se, $I-P$ é uma projeçã projeção compacta. Portanto, sendo tal projeção compacta20, então ela possui núcleo ou imagem de dimensão finita, contrariando a decomponibilidade de $X_{A H}{ }^{21}$

Como $X_{A H}$ não possui uma base incondicional - apenas base de Schauder - então obviamente não podemos utilizar o Teorema 4.21 diretamente em tal espaço. Como fizemos em outros momentos, nos resta inspecionar o contraexemplo feito por Bayart e Matheron em [7], verificar o quão essencial a hipótese da base ser incondicional é e se podemos adaptar tal resultado para o espaço $X_{A H}$.

Ao analisarmos a construção de Bayart e Matheron, vemos que a hipótese da incondicionalidade da base surge apenas para mostrar que o operador $T$ construído para ser o contraexemplo é contínuo, e não na construção do operador em si (vide o Corolário 3.2 em [7]). Logo, podemos replicar a construção normalmente em $X_{A H}$, apenas com a ressalva de demonstrar a continuidade de $T$ de outra maneira.

Como a construção original de Bayart e Matheron é feita sob a hipótese do espaço de Banach ser qualquer - e com isso a sua norma também é qualquer - surge a esperança de que ao lidarmos com $X_{A H}$, que é um espaço cuja norma é conhecida (a saber, a norma do supremo $\|\cdot\|_{\infty}$ ), então o conhecimento de tal norma seja suficiente para mostrar que o operador $T$ é contínuo. Em outras palavras, aparece a ideia de que talvez a incondicionalidade da base seja uma hipótese feita apenas para "superar" o desconhecimento da norma do espaço em questão (e que, em casos que conhecemos a norma, tal incondicionalidade não se faria necessária para mostrar a continuidade de $T$ ).

Apesar de tal ideia ser promissora e "fazer sentido", é possível mostrar que o operador $T$ construído por Bayart e Matheron não pode ser contínuo em $X_{A H}$. E curiosamente veremos que o motivo de tal operador não poder ser contínuo em $X_{A H}$ é justamente o que nos atraiu a estudar tal espaço em primeiro lugar: o fato dele possuir pouquíssimos operadores. Para mostramos que tal $T$ não pode ser contínuo, vamos estudar sua construção.

Seja $\left(b_{n}\right)_{n=1}^{\infty}$ uma sequência crescente de números naturais. Se $\left(e_{n}\right)_{n=0}^{\infty}$ é uma base normalizada de $X_{A H}$, definimos $T$ da seguinte maneira:

$$
T\left(e_{i}\right)= \begin{cases}2 e_{i+1} & \text { se } i \in\left[b_{n-1}, b_{n}-1[, \text { para algum } n \geq 1\right. \\ \varepsilon_{n} e_{b_{n}}+f_{n} & \text { se } i=b_{n}-1, \text { para algum } n \geq 1\end{cases}
$$

\footnotetext{
${ }^{19}$ Para uma referência neste resultado, ver [22, Teorema 3.2.7, p. 297].

${ }^{20}$ Note que se obtemos $\lambda=0$ anteriormente, então $P$ é compacto; no caso de obtermos $\lambda=1$, então $I-P$ é uma projeção compacta.

${ }^{21}$ Não dissemos, mas $X_{A H}$ também é hereditariamente indecomponível (HI), isto é, todo $M \subseteq X_{A H}$ subespaço de dimensão infinita é indecomponível. É claro que como $X_{A H}$ é HI, então $X_{A H}$ não pode ter base incondicional.
} 
onde $\varepsilon_{n} \in \mathbb{K}$ e $f_{n} \in X_{A H}$.

A definição precisa de $\varepsilon_{n}$ e de $f_{n}$ não é relevante no nosso contexto. A partir de agora, considere a subsequência $\left(e_{j_{k}}\right)_{k=1}^{\infty} \subseteq\left(e_{n}\right)_{n=0}^{\infty}$ tal que $j_{k} \neq b_{n}-1$, para todo $n \geq 1$; isto é, estamos retirando da sequência $\left(e_{n}\right)_{n=0}^{\infty}$ os elementos de índice $b_{n}-1$.

Suponha que $T$ seja contínuo em $X_{A H}$. Como $X_{A H}$ tem pouquíssimos operadores, então podemos escrever $T=\lambda I+K$, para algum $\lambda \in \mathbb{K}$ e algum operador $K$ compacto. Vamos mostrar que a sequência limitada ${ }^{22}\left(e_{j_{k}}\right)_{k=1}^{\infty}$ é tal que $\left(K e_{j_{k}}\right)_{k=1}^{\infty}$ não possui uma subsequência convergente, uma contradição com a compacidade de $K$.

Temos que $K e_{j_{k}}=(T-\lambda I)\left(e_{j_{k}}\right)=2 e_{j_{k}+1}-\lambda e_{j_{k}}$, uma vez que $j_{k} \neq b_{n}-1$. Antes de prosseguirmos, vamos precisar de uma proposição sobre bases em espaços de Banach:

Proposição 4.22 (Teorema 4.1.14 de [22]). Seja $\left(x_{n}\right)_{n \in \mathbb{N}}$ uma base de Schauder para o espaço de Banach $(X,\|\cdot\|)$. Defina $\|\cdot\|_{\left(x_{n}\right)}$ em $X$ por

$$
\|x\|_{\left(x_{n}\right)}=\sup _{k}\left\|\sum_{n=1}^{k} \alpha_{n} x_{n}\right\|
$$

onde $x=\sum_{n=1}^{\infty} \alpha_{n} x_{n}$. Então $\|\cdot\|_{\left(x_{n}\right)}$ é uma norma equivalente a $\|\cdot\|$ e tal que $\|x\|_{\left(x_{n}\right)} \geq\|x\|$, para todo $x \in X$.

De posse desta proposição, podemos mostrar que $\left(K e_{j_{k}}\right)_{k=1}^{\infty}$ não possui uma subsequência convergente. Seja $\left(K e_{j_{k}}\right)_{i=1}^{\infty} \subseteq\left(K e_{j_{k}}\right)_{k=1}^{\infty}$ uma subsequência qualquer. Se $\lambda \neq 0$, temos que:

$$
\begin{aligned}
\left\|K e_{j_{k_{i}}}-K e_{j_{k_{l}}}\right\| & \geq M\left\|K e_{j_{k_{i}}}-K e_{j_{k_{l}}}\right\|_{\left(e_{n}\right)}=M\left\|2 e_{j_{k_{i}}+1}-\lambda e_{j_{k_{i}}}-2 e_{j_{k_{l}}+1}+\lambda e_{j_{k_{l}}}\right\|_{\left(e_{n}\right)} \\
& \geq M\left\|-\lambda e_{j_{k_{i}}}+2 e_{j_{k_{i}}+1}+\lambda e_{j_{k_{l}}}-2 e_{j_{k_{l}}+1}\right\|_{\left(e_{n}\right)} \\
& \geq M|\lambda|
\end{aligned}
$$

para $i>l$. Se $\lambda=0$, teríamos então que

$$
\left\|K e_{j_{k_{i}}}-K e_{j_{k_{l}}}\right\| \geq M\left\|2 e_{j_{k_{i}}+1}-2 e_{j_{k_{l}}+1}\right\|_{\left(e_{n}\right)} \geq 2 M
$$

De qualquer maneira, concluímos que $\left\|K e_{j_{k_{i}}}-K e_{j_{k_{l}}}\right\| \nrightarrow \nrightarrow 0$ e, portanto, tal subsequência não converge.

Mostrado então que não podemos adaptar fielmente a construção de Bayart e Matheron no espaço $X_{A H}$, temos uma observação importante para fazer:

Observação 4.23. Em trabalhos seguintes, Bayart e Matheron simplificaram a construção original feita em [7]. Na Seção 4.2 de [6], Bayart e Matheron apresentam a construção com uma leve modificação: para o caso em que $i \in\left[b_{n-1}, b_{n}-1\right.$ [, ao invés de definir $T\left(e_{i}\right)=2 e_{i+1}$, eles adotam

\footnotetext{
${ }^{22}$ Tal sequência é limitada pois estamos tomando $\left(e_{n}\right)_{n=0}^{\infty}$ como sendo uma base normalizada.
} 
$T\left(e_{i}\right)=w(i+1) e_{i+1}$, onde $(w(i))_{i=1}^{\infty}$ é uma sequência crescente de números positivos tais que

$$
w(i):=4\left(1-\frac{1}{2 \sqrt{i}}\right)
$$

para todo $i \in \mathbb{N}$. Isto faz com que a definição de $T$ apresentada em [7] (e que exibimos na Equação (4.4) ) seja um caso particular desta: basta tomar $w(i)=2$, para todo $i \in \mathbb{N}$.

Definindo $T$ dessa maneira, evidentemente que a demonstração da não-continuidade de $T$ feita acima muda. No entanto, não é difícil observar que a única mudança relevante seria na Equação 4.5): obteríamos

$$
\left\|K e_{j_{k_{i}}}-K e_{j_{k_{l}}}\right\| \geq w\left(j_{k_{l}}\right) M
$$

Como $(w(i))_{i=1}^{\infty}$ é uma sequência crescente e tal que $w(i) \rightarrow 4$, então a conclusão continua válida.

Em face desta última equação, é natural perguntar: não poderíamos trocar a sequência $(w(i))_{i=1}^{\infty}$ por uma sequência decrescente tal que $w(i) \rightarrow 0$ ? Isto eliminaria a contradição obtida na Equação 4.6) (uma vez que seria possível encontrar subsequências convergentes) e continuaria deixando em aberto a possibilidade de $T$ ser contínuo ou não.

Ao analisar mais uma vez a construção de Bayart e Matheron, fica claro que tomar $(w(i))_{i=1}^{\infty}$ decrescente e tal que $w(i) \rightarrow 0$ não é possível. O motivo é simples: a escolha de $(w(i))_{i=1}^{\infty}$ crescente e tal que $2 \leq w(i) \leq 4$ nos permite concluir que as sequências $\left(\varepsilon_{n}\right)_{n=1}^{\infty}$ e $\left(f_{n}\right)_{n=1}^{\infty}$ usadas na definição de $T$ sejam limitadas. Ao tomarmos $(w(i))_{i=1}^{\infty}$ decrescente e tal que $w(i) \rightarrow 0$, inevitavelmente uma das duas sequências deixa de ser limitada. Assim, chegamos a mesma conclusão de que $T$ não é contínuo: nesse caso, através da expressão $T\left(e_{b_{n}-1}\right)=\varepsilon_{n} e_{b_{n}}+f_{n}$ (vide a segunda linha da Equação (4.4) 23

Como pode-se perceber, o argumento que usamos para mostrar que a construção de Bayart e Matheron não pode ser replicada em $X_{A H}$ pode ser utilizado em qualquer espaço com pouquíssimos operadores, entre eles os espaços $X_{\mathfrak{n} r}$ e $X_{K u s}$ mencionados na introdução do capítulo. É interessante ressaltar que apesar do espaço $X_{K u s}$ não possuir base incondiciona ${ }^{24}$, ele é saturado com sequências básicas incondicionais: isto é, todo subespaço $Y \subseteq X_{K u s}$ de dimensão infinita possui uma sequência básica incondicional. Apesar de possuir essa propriedade - e, intuitivamente, ser um espaço mais apropriado para replicar a construção de Bayart e Matheron - o fato dele possuir pouquíssimos operadores inviabiliza tal estratégia, como vimos nesta seção.

Dessa forma, para construirmos um exemplo de um operador hipercíclico que não satisfaz o Critério de Hiperciclicidade em espaços com pouquíssimos operadores, precisamos pensar em construções diferentes daquela feita por Bayart e Matheron. Este é o objetivo de pesquisas futuras.

Por fim, vale ressaltar que, se tentarmos reproduzir a construção de Bayart e Matheron em um espaço em que conhecemos a norma, a hipótese da base ser incondicional pode sim ser substituída

\footnotetext{
${ }^{23} \mathrm{O}$ fato de que $2 \leq w(i) \leq 4$ não é tão crucial assim na construção de Bayart e Matheron: poderíamos escolher $(w(i))_{i=1}^{\infty}$ crescente e tal que $0<a \leq w(i) \leq b$ e a construção continuaria bastante similar. No entanto, o fato de $(w(i))_{i=1}^{\infty}$ ser crescente é muito mais importante, ao ponto de que se tomássemos uma sequência descrescente (ainda que $w(i) \rightarrow a>0)$, a construção precisaria ser bastante modificada.

${ }^{24}$ Como argumentamos anteriormente, nenhum espaço com pouquíssimos operadores pode ter base incondicional.
} 
por outra que também é suficiente para mostrar que $T$ é contínuo:

Teorema 4.24. Seja $(X,\|\cdot\|)$ um espaço de Banach. Suponha que X admita uma base de Schauder normalizada $\left(e_{i}\right)_{i=0}^{\infty}$. Denote $E=\operatorname{span}\left\{e_{i}: i=0,1,2 \ldots\right\}$. Suponha que $\|\cdot\|$ seja tal que, para toda sequência $\left(\alpha_{n}\right)_{n \in \mathbb{N}}$ tal que $\sup _{n \in \mathbb{N}}\left|\alpha_{n}\right|<\infty$, o operador $S: E \rightarrow E$ dado por $S\left(e_{i}\right)=\alpha_{i+1} e_{i+1}$ é contínuo. Então existe um operador hipercíclico $T \in \mathcal{B}(X)$ tal que $T \oplus T$ não é hipercíclico (e, portanto, $T$ não satisfaz o Critério de Hiperciclicidade).

Não vamos entrar em detalhes de como esta substituição da hipótese ocorre uma vez que tal tarefa foge do escopo deste texto. Para verificar que isto é possível, sugerimos a leitura da Seção 4.2 e da Proposição 4.21 de [6].

\subsection{Sub-Ciclicidade}

Uma vez que estudamos sub-hiperciclicidade, é natural tentarmos estender o conceito de "densidade em subespaços" para outros fenômenos similares, como ciclicidade. Com isso em mente, nesta seção vamos definir o conceito de sub-ciclicidade.

Como é de se imaginar, os resultados envolvendo sub-ciclicidade serão bastante parecidos com os que já vimos para sub-hiperciclicidade. No entanto, do mesmo jeito que existem diferenças entre hiperciclicidade e ciclicidade, também existem diferenças entre os conceitos equivalentes para subespaços. Veremos, por exemplo, que o Teorema 4.31 não admite um equivalente para operadores sub-hipercíclicos. Formularemos também um Critério de Sub-Ciclicidade e daremos um exemplo de um operador sub-cíclico que não satisfaz tal critério.

Vamos definir o conceito de sub-ciclicidade:

Definição 4.25. Seja $X$ um espaço de Banach e $T \in \mathcal{B}(X)$. Dizemos que $T$ é sub-cíclico se existe um $x \in X$ e um subespaço fechado de dimensão infinita não-trivia 25 tal que $[\operatorname{span}$ orb $(x, T)] \cap M$ é denso em $M$. Neste caso, também dizemos que $T$ é $M$-cíclico ou sub-cíclico para $M$ e que $x$ é um vetor $M$-cíclico para $\mathbf{T}$.

É imediato constatar que todo operador sub-hipercíclico também é sub-cíclico. Além disso, observe que na definição acima utilizamos $A:=[\operatorname{span} \operatorname{orb}(x, T)] \cap M$ ao invés de $B:=\operatorname{span}[\operatorname{orb}(x, T) \cap$ $M]{ }_{{ }^{26}}^{2 a}$ Não é difícil mostrar que $B \subseteq A$; no entanto, em geral não necessariamente vale que $A \subseteq B$. Vejamos no simples exemplo a seguir um caso em que $A \nsubseteq B$ :

Exemplo 4.26. Seja $F$ o já visto forward shift em $\ell_{p}(1 \leq p<\infty)$. Seja $x=(1,1,0,0, \ldots)=e_{1}+e_{2}$ e considere $M=\left\{\left(x_{n}\right)_{n \in \mathbb{N}} \in \ell_{p}: x_{2 n}=0, n \in \mathbb{N}\right\}$. É claro que $M$ é fechado e tem dimensão infinita. Como $F e_{n}=e_{n+1}$ para todo $n \in \mathbb{N}$, temos que que $F^{n} x=e_{n+1}+e_{n+2}$, para todo $n \in \mathbb{N}$. Logo, não é difícil ver que orb $(x, F) \cap M=\emptyset \mathrm{e}$, portanto, $B=\operatorname{span}[\operatorname{orb}(x, F) \cap M]=\emptyset$.

Agora observe que $x-F x=e_{1}+e_{2}-\left(e_{2}+e_{3}\right)=e_{1}-e_{3}$. Portanto, temos que $x-F x \in M$ e, como $x-F x \in \operatorname{span}$ orb $(x, F)$, segue que $x-F x \in[\operatorname{span}$ orb $(x, F)] \cap M$. Dessa forma, temos que $A=[\operatorname{span} \operatorname{orb}(x, F)] \cap M \neq \emptyset$. Como $B=\emptyset$, segue então que $A \nsubseteq B$, como queríamos mostrar.

\footnotetext{
${ }^{25}$ Isto é, $M \neq X$.

${ }^{26}$ Mesmo se tivéssemos utilizado o conjunto $B:=\operatorname{span}[\operatorname{orb}(x, T) \cap M]$ para definir a noção de sub-ciclicidade, continuaria valendo que todo operador sub-hipercíclico também é sub-cíclico.
} 
Dito isso, devemos então justificar o porquê de utilizarmos o conjunto $A$ ao invés do conjunto $B$ na definição anterior. Como veremos adiante, os resultados de sub-hiperciclicidade se traduzem naturalmente para sub-ciclicidade ao usarmos a definição dada. Além disso, acreditamos que tal definição é mais "natural": quando estudamos sub-hiperciclicidade, estamos interessados em procurar (pelo menos) um subespaço de maneira que a interseção de tal subespaço com uma orb $(x, T)$ seja denso no subespaço, uma vez que o conjunto que nos interessa ao estudarmos hiperciclicidade é justamente a órbita de um vetor pelo operador. No caso de sub-ciclicidade, é natural que o conjunto escolhido para interseccionar o subespaço seja span orb $(x, T)$, uma vez que esse é justamente o conjunto de interesse quando estudamos ciclicidade.

No caso de sub-hiperciclicidade, mostramos que a existência de um vetor $x$ tal que orb $(x, T)$ seja linearmente independente era uma condição necessária (Lema 2.9) mas não suficiente (Exemplo 2.10. É natural, tendo em mente a definição que escolhemos para sub-ciclicidade, que a condição continue sendo necessária, como mostra a próxima proposição:

Proposição 4.27. Seja $T$ um operador $M$-cíclico. Se $x$ é um vetor $M$-cíclico para $T$, então orb $(x, T)$ é um conjunto infinito e linearmente independente.

DemonstraÇão: A demonstração é análoga à do Lema 2.9. De maneira a deixar o texto completo, vamos escrevê-la. Suponha que orb $(x, T)$ seja linearmente dependente. Temos então que existem $k \in \mathbb{N}$ e escalares $a_{1}, \ldots, a_{k-1}$ não todos nulos tais que

$$
T^{k}(x)=\sum_{j=1}^{k-1} a_{j} T^{j} x
$$

Dessa forma, temos que se $A=\operatorname{span}\left\{T^{j}(x): 1 \leq j \leq k-1\right\}$, então $T^{k}(x) \in A$ e portanto

$$
T^{k+1}(x)=T\left(T^{k} x\right)=T\left(\sum_{j=1}^{k-1} a_{j} T^{j} x\right)=\sum_{j=1}^{k-1} a_{j} T^{j+1} x \in A
$$

uma vez que $A$ é um subespaço. Indutivamente, segue que $T^{j} x \in A$, para todo $j \in \mathbb{N}$. Portanto, temos que span orb $(x, T)=A$.

Sendo $T$ um operador $M$-cíclico, então $[\operatorname{span} \operatorname{orb}(x, T)] \cap M=A \cap M$ é denso em $M$. Note agora que, como $A \cap M \subseteq A$ e $\operatorname{dim} A<\infty$, então é claro que $A \cap M$ tem dimensão finita. No entanto, isto é um absurdo, tendo em mente que $A \cap M$ deve ser denso em $M$, um subespaço fechado de dimensão infinita.

Como dito anteriormente, no caso de sub-hiperciclicidade a condição "existe um vetor $x$ tal que orb $(x, T)$ seja linearmente independente" não é suficiente. Surge, então, a pergunta natural: no caso de sub-ciclicidade, será tal condição suficiente ou não? Os próximos resultados responderão essa pergunta.

Proposição 4.28. Sejam $X$ um espaço de Banach e $T \in \mathcal{B}(X)$. Suponha que $T$ não seja cíclico e exista um vetor $x \in X$ tal que orb $(x, T)$ seja infinito e linearmente independente. Então $T$ é sub-cíclico. 
Demonstração: Seja $x \in X$ tal que orb $(x, T)$ seja linearmente independente. Além disso, como $T$ não é cíclico, então $M_{x}=\overline{\operatorname{spanorb}(x, T)}$ é tal que $M_{x} \neq X$. Como escolhemos o $x \in X$ de maneira a termos orb $(x, T)$ infinito e linearmente independente, então $M_{x}$ tem dimensão infinita (e, portanto, é não-trivial).

Agora, não é difícil ver que span orb $(x, T) \cap M_{x}=\operatorname{span}$ orb $(x, T)$ é denso em $M_{x}$, o que mostra que $T$ é $M_{x}$-cíclico.

Observe que, mesmo se utilizássemos span $[\operatorname{orb}(x, T) \cap M]$ na definição de sub-ciclicidade, a construção acima ainda seria válida uma vez que, pela construção de $M_{x}, \operatorname{span}\left[\operatorname{orb}(x, T) \cap M_{x}\right]=$ $[\operatorname{span}$ orb $(x, T)] \cap M_{x}=\operatorname{span}$ orb $(x, T)$. Note ainda que o fato de $T$ não ser cíclico foi importante para a construção do subespaço para o qual $T$ é $M$-cíclico.

Uma vez demonstrada a proposição anterior, para provarmos que a condição "existe um $x$ tal que orb $(x, T)$ seja linearmente independente" é suficiente, temos agora que considerar o caso em que $T$ é cíclico. No entanto, note que ao supor $T$ cíclico, a existência de um $x$ tal que orb $(x, T)$ seja linearmente independente é garantida, uma vez que deve existir um $x$ tal que $\operatorname{span} \operatorname{orb}(x, T)$ é denso em $X$. Dessa maneira, por questões de elegância, deixamos tal hipótese de fora da próxima proposição:

Proposição 4.29. Sejam $X$ um espaço de Banach e $T \in \mathcal{B}(X)$. Se $T$ é cíclico, então $T$ é subcíclico.

Demonstração: Seja $x$ um vetor cíclico para $T$. Então span orb $(x, T)$ é denso em $X$. Logo, pelo Teorema 2.6, existe um subespaço fechado $M$ de dimensão infinita tal que $[\operatorname{span} \operatorname{orb}(x, T)] \cap M$ é denso em $M$. Isto mostra que $T$ é $M$-cíclico.

Vale ressaltar que, ao definirmos sub-ciclicidade usando $[\operatorname{span} \operatorname{orb}(x, T)] \cap M$ ao invés de $\operatorname{span}[\operatorname{orb}(x, T) \cap M]$, demonstrar a proposição acima ficou claramente mais fácil. Assim, juntando as duas proposições anteriores obtemos o seguinte resultado:

Proposição 4.30. Seja $X$ um espaço de Banach e $T \in \mathcal{B}(X)$. Se existir um vetor $x$ tal que orb $(x, T)$ seja infinito e linearmente independente, então $T$ é sub-cíclico.

Por fim, juntando a proposição acima com a Proposição 4.27, obtemos o resultado desejado:

Teorema 4.31. Seja $X$ um espaço de Banach e $T \in \mathcal{B}(X)$. Então $T$ é sub-cíclico se, e somente se, existir um vetor $x$ tal que orb $(x, T)$ seja infinito e linearmente independente.

De posse deste resultado, é mais simples obter exemplos e mostrar que certos operadores são sub-cíclicos, como veremos a seguir:

Exemplo 4.32. Seja $F$ o já conhecido forward shift, só que desta vez considere $F \in \mathcal{B}\left(\ell_{\infty}\right)$. Não é difícil verificar que orb $\left(e_{1}, F\right)$ é infinito e linearmente independente. Portanto, $F$ é sub-cíclico em $\ell_{\infty}$, embora não seja um operador cíclico em $\ell_{\infty} 2^{27}$

\footnotetext{
${ }^{27}$ Uma vez que $\ell_{\infty}$ não é separável.
} 
Como $\|F\|=1$, podemos concluir também $F$ não é um operador sub-hipercíclico (vide o Lema 2.11) e nem hipercíclico (vide o Teorema 2.7).

Como todo operador sub-hipercíclico é evidentemente sub-cíclico, então muitos dos resultados que colocamos nos capítulos anteriores também valem para operadores sub-cíclicos. Logo, em todo espaço de Banach existe um operador sub-cíclico (baseado no que vimos no Teorema 2.31), dado um subespaço separável de dimensão infinita $M$, existe um operador $M$-cíclico (visto no Teorema 2.32 , um operador $T$ que é $M$-invariante é $M$-cíclico se, e somente se, $\left.T\right|_{M}$ for cíclico (Observação $2.4)$ e outros resultados envolvendo quasi-conjugações.

Dito isso, não vamos refazer e adaptar tais resultados para operadores sub-cíclicos, uma vez que os enunciados e suas demonstrações envolvidas seriam totalmente análogas. No entanto, vale a pena adaptarmos para operadores sub-cíclicos um resultado importante: o Critério de Ciclicidade.

Antes de apresentá-lo, vale dizer que em quase todos os critérios que apresentamos (a saber, o HC, o CC e o SHC) usamos alguma noção de transitividade para demonstrar o critério em questão ${ }^{28}$ Para demonstrarmos o Critério de Sub-Ciclicidade, não será diferente: vamos então definir uma noção de transitividade para ciclicidade em subespaços (similar ao que foi feito na Definição 3.1) e demonstrar que ela implica em sub-ciclicidade.

Proposição 4.33. Sejam $X$ um espaço de Banach, $T \in \mathcal{B}(X)$ e $M \subsetneq X$ um subespaço fechado, separável e de dimensão infinita. Suponha que, dados $U, V$ abertos não-vazios de $M$, existe um $p \in \mathbb{K}[x]$ tal que $[p(T)]^{-1}(U) \cap V$ é um aberto não-vazio de $M$. Então $T$ é $M$-cíclico.

Observação 4.34. Antes de apresentar a demonstração, vamos esclarecer um pouco a linguagem. Quando dissemos que $U$ é um aberto de $M$, queremos dizer que $U \subseteq M$ é tal que $U=U_{1} \cap M$, para algum aberto $U_{1}$ de $X$ (ou seja, estamos evidentemente considerando a topologia de subespaço em $M)$.

Seguiremos agora com a demonstração da última proposição:

DemonstraÇão: Vamos proceder de maneira parecida ao que fizemos na Proposição 4.4. Denote por $C(T)$ o conjunto dos vetores $M$-cíclicos para $T$. Vamos mostrar que $C(T)$ é não-vazio, o que evidentemente mostra que $T$ é $M$-cíclico.

Sendo $M$ separável, temos que $M$ admite uma base enumerável de abertos $\left(U_{k}\right)_{k \in \mathbb{N}} \subseteq M$. Por hipótese, dados $j, k \in \mathbb{N}$, existe um $p_{j, k} \in \mathbb{K}[x]$ tal que $\left[p_{j, k}(T)\right]^{-1}\left(U_{j}\right) \cap U_{k}$ é um aberto não-vazio de $M$. Logo, o conjunto

$$
A_{j}:=\bigcup_{k=1}^{\infty}\left[p_{j, k}(T)\right]^{-1}\left(U_{j}\right) \cap U_{k}
$$

\footnotetext{
${ }^{28}$ No Critério de Hiperciclicidade, usamos o Teorema da Transitividade de Birkhoff (Teorema 1.17 No Critério de Sub-Hiperciclicidade, usamos a noção de $M$-transitividade (Definição 3.1) e o Teorema 3.2 Já no Critério de Ciclicidade utilizamos o Teorema 4.4 .
} 
é um aberto não-vazio de $M$. Ainda, temos que cada $A_{j}$ é denso em $M$, uma vez que a interseção de $A_{j}$ com cada $U_{k} \in\left(U_{k}\right)_{k \in \mathbb{N}}$ é não-vazia (por hipótese).

Pelo Teorema de Baire, temos que $\bigcap_{j=1}^{\infty} A_{j}$ é denso em $M$ - e, portanto, não-vazio. Para terminar a demonstração, observe que $x \in C(T)$ se, e somente se, dado $j \in \mathbb{N}$, existe $p \in \mathbb{K}[x]$ tal que $p(T) x \in U_{j} \subseteq M$. Ou seja,

$$
C(T)=\bigcap_{j=1}^{\infty} \bigcup_{p \in \mathbb{K}[x]} p(T)^{-1}\left[U_{j}\right] \supseteq \bigcap_{j=1}^{\infty} \bigcup_{k=1}^{\infty} p_{j, k}(T)^{-1}\left[U_{j}\right] \cap U_{k}=\bigcap_{j=1}^{\infty} A_{j}
$$

e como o conjunto mais à direita é não-vazio, segue que $C(T)$ é não-vazio, como desejado.

Provada a proposição anterior, podemos agora enunciar e demonstrar um Critério de SubCiclicidade. Como é de se imaginar, o enunciado de tal critério é baseado no Critério de Ciclicidade e sua adaptação para subespaços é baseada no Critério de Sub-Hiperciclicidade. A demonstração que daremos é totalmente baseada na demonstração original do SHC (feita em [18, Teorema 3.6, pp. 505-506]).

Teorema 4.35 (Critério de Sub-Ciclicidade). Sejam $X$ um espaço de Banach, $T: X \rightarrow X$ um operador linear limitado e $M$ um subespaço fechado separável de dimensão infinita de $X$ tal que $M \neq X$. Suponha que existem subconjuntos $X_{0}, Y_{0}$ densos em $M$ e uma sequência de polinômios $\left(p_{n}\right)_{n \in \mathbb{N}} \subseteq \mathbb{K}[x]$ tais que:

(i) $p_{n}(T)(x) \rightarrow 0$, para todo $x \in X_{0}$.

(ii) para cada $y \in Y_{0}$, existe uma sequência $\left(x_{n}\right)_{n \in \mathbb{N}} \subseteq M$ tal que

$$
x_{n} \rightarrow 0 \quad \text { e } \quad p_{n}(T) x_{n} \rightarrow y
$$

(iii) $M$ é um subespaço invariante para $p_{n}(T)$, para todo $n \in \mathbb{N}$

Então T é $M$-cíclico.

DemonstraÇÃo: Vamos mostrar que, dados $U, V$ abertos não-vazios de $M$, existe um $p \in \mathbb{K}[x]$ tal que $[p(T)]^{-1}(U) \cap V$ é um aberto não-vazio de $M$. Assim, pela Proposição 4.33 , seguirá que $T$ é $M$-cíclico.

Tome, então, $U, V$ abertos não-vazios de $M$. Pela densidade de $X_{0}$ e $Y_{0}$ em $M$, podemos achar $x \in X_{0} \cap V \subseteq M$ e $y \in Y_{0} \cap U \subseteq M$. Ainda, como $U, V$ são abertos em $M$, existe um $\varepsilon>0$ tal que $B(x, \varepsilon) \cap M \subseteq V$ e $B(y, \varepsilon) \cap M \subseteq U$.

Como $x \in X_{0}$ e $y \in Y_{0}$, usando as condições (i) e (ii) podemos achar um $k \in \mathbb{N}$ e $x_{k} \in M$ tal que $\left\|p_{k}(T) x\right\|<\varepsilon / 2,\left\|x_{k}\right\|<\varepsilon$ e $\left\|p_{k}(T) x_{k}-y\right\|<\varepsilon / 2$.

Agora, como $x, x_{k} \in M$ segue que $x+x_{k} \in M$. Vamos então mostrar que $x+x_{k} \in V$ e $p_{k}(T)\left(x+x_{k}\right) \in U$. Evidentemente isto mostrará que $\left[p_{k}(T)\right]^{-1}(U) \cap V \neq \emptyset$.

Para mostrar que $x+x_{k} \in V$, note que $\left\|\left(x+x_{k}\right)-x\right\|=\left\|x_{k}\right\|<\varepsilon$, donde obtemos que $x+x_{k} \in B(x, \varepsilon) \cap M \subseteq V$, como desejado. 
Para mostrar a segunda parte, observe que pela condição (iii) temos que $p_{k}(T)\left(x+x_{k}\right) \in$ $M$. Ainda, de maneira similar ao que fizemos anteriormente, temos que $\left\|p_{k}(T)\left(x+x_{k}\right)-y\right\| \leq$ $\left\|p_{k}(T) x\right\|+\left\|p_{k}(T) x_{k}-y\right\|<\varepsilon / 2+\varepsilon / 2=\varepsilon$, o que mostra que $p_{k}(T)\left(x+x_{k}\right) \in B(y, \varepsilon) \cap M \subseteq U$. Logo, $x+x_{k} \in\left[p_{k}(T)\right]^{-1}(U)$, como queríamos.

Mostramos que $\left[p_{k}(T)\right]^{-1}(U) \cap V \neq \emptyset$. Resta mostrar que tal conjunto é aberto em $M$. Por (iii), o operador $\left.p_{k}(T)\right|_{M}: M \rightarrow M$ está bem definido e é contínuo (uma vez que $T$ o é). Logo, como $U$ é um aberto de $M$, então $\left[\left.p_{k}(T)\right|_{M}\right]^{-1}(U)$ é um aberto de $M$. Como $V$ é um aberto de $M$, então $\left[\left.p_{k}(T)\right|_{M}\right]^{-1}(U) \cap V=\left[p_{k}(T)\right]^{-1}(U) \cap V$ é aberto em $M$, como desejado.

Indo ao encontro do que fizemos em todos os outros critérios apresentados, quando necessário faremos referência ao Critério de Sub-Ciclicidade pela sigla $S C C$ e quando $T$ satisfazer o Critério de Sub-Ciclicidade para um certo subespaço $M$, diremos que $T$ satisfaz o SCC para M.

Da mesma maneira que resultados para operadores sub-hipercíclicos se traduzem de maneira bem natural para operadores sub-cíclicos (como comentamos anteriormente), a dinâmica da relação entre o Critério de Ciclicidade e o Critério de Sub-Ciclicidade é análoga àquela entre o HC e o SHC. Por exemplo, o Lema 3.5 se traduz naturalmente para o caso cíclico:

Lema 4.36. Sejam $X$ um espaço de Banach de dimensão infinita, $T: X \rightarrow X$ um operador linear limitado e $M$ um subespaço fechado separável de dimensão infinita de $X$ tal que $M \neq X$. Suponha que $T$ seja $M$-invariante e considere o operador $\left.T\right|_{M}: M \rightarrow M$. Então $\left.T\right|_{M}$ satisfaz o CC se, e somente se, $T$ satisfaz o SCC para $M$.

DemonstraÇÃo: Trivial.

Ainda, como supomos que $i$ é linear nos resultados envolvendo o SHC e quasi-conjugações vistos na Seção 3.3 (a saber, as Proposições 3.16 e 3.17), então tais resultados também se traduzem naturalmente para ciclicidade e sub-ciclicidade (uma vez que, se $i$ é linear e $S \circ i=i \circ T$, então vale que $p(S) \circ i=i \circ p(T)$, para todo $p \in \mathbb{K}[x]$ ). Sabendo disso e de posse do último lema, podemos facilmente dar exemplos de operadores sub-cíclicos que não satisfazem o SCC: basta começarmos com operadores cíclicos que não satisfazem o CC (por exemplo, aqueles vistos nos Exemplos 4.5 e 4.11).

Exemplo 4.37. Sejam $X$ um espaço de Banach separável e $T \in \mathcal{B}(X)$ um operador cíclico que não satisfaz o CC. Considere $i: X \rightarrow \ell_{\infty}$ uma imersão isométrica (via o Teorema 1.6). Defina $\tilde{T}: i(X) \rightarrow \ell_{\infty}$ por $\tilde{T} i(x)=i(T x)$. Como $i$ é uma imersão isométrica, então $\tilde{T}$ está bem definido. Além disso, como $\ell_{\infty}$ é isometricamente injetivo (vide a Proposição 1.11), então podemos estender $\tilde{T}$ à $\ell_{\infty}$; para simplificar, vamos cometer o abuso de denotar tal extensão também por $\tilde{T}$.

Pela definição, temos que $\tilde{T} \circ i=i \circ T$. Logo, como $T$ é cíclico e não satisfaz o CC, então pelo que discutimos anteriormente podemos concluir que $\tilde{T}$ é $i(X)$-cíclico mas não satisfaz o SCC. 
É trivial observar que todo operador que satisfaz o SHC também satisfaz o Critério de SubCiclicidade. Assim, não nos falta exemplos de operadores que satisfazem o SCC. No entanto, achar um contraexemplo para o SCC pode ser difícil: apesar de já termos visto exemplos de operadores sub-hipercíclicos que não satisfazem o SHC (vide o Exemplo 3.7), não necessariamente um operador que não satisfaz o SHC também não satisfaz o SCC (da mesma maneira que, como vimos no capítulo anterior, existem operadores que satisfazem o CC mas não o HC). Por isso o exemplo descrito acima é importante.

Além deste exemplo, vamos agora apresentar um outro para mostrar que a noção de transitividade para operadores cíclicos em subespaços apresentada na Proposição 4.33 não é uma condição necessária para sub-ciclicidade - indo de encontro ao que acontece com sub-hiperciclicidade. Como tal caso já acontece em sub-hiperciclicidade, vamos então utilizar o mesmo operador e subespaço que foram utilizados em um dos exemplos feitos para mostrar tal fenômeno (a saber, o Exemplo 3.8 de [18]) para mostrar que o mesmo ocorre em sub-ciclicidade. No que segue, vamos denotar por $\operatorname{deg} p$ o grau do polinômio $p$ e por $e_{j}$ o vetor canônico de $\ell_{2}$.

Exemplo 4.38. Seja $X=\ell_{2}$ e fixe um $m>1$. Considere $M:=\left\{\left(x_{n}\right)_{n=1}^{\infty} \in \ell_{2}: x_{n}=0\right.$ se $\left.n<m\right\}$. Seja $\lambda \in \mathbb{K}$ tal que $|\lambda|>1$. No Exemplo 3.8 de [18], foi mostrado que $\lambda B$, onde $B$ é o conhecido backward shif 29 é $M$-hipercíclico mas não é $M$-transitivo. Dessa forma, é claro que $\lambda B$ é $M$-cíclico. Vamos mostrar que não existe $p \in \mathbb{K}[x] \operatorname{com} \operatorname{deg} p>0$ tal que $[p(\lambda B)]^{-1}(U) \cap V$ é um aberto nãovazio de $M{ }^{30}$ Para isso, vamos mostrar que, se tal $p$ existir, teremos então que $p(\lambda B)(M) \subseteq M$, o que é claramente um absurdo 31

Suponha então que tal $p \in \mathbb{K}[x]$ exista. Seja $x \in M$ qualquer. Vamos mostrar que $p(\lambda B) x \in M$. Para isso, denote por $W=[p(\lambda B)]^{-1}(U) \cap V$. Logo, é claro que $W \subseteq[p(\lambda B)]^{-1}(U) \Rightarrow p(\lambda B)(W) \subseteq$ $U \subseteq M$. Tome $x_{0} \in W$. Como $W$ é um aberto de $M$ e $x \in M$, então podemos tomar um $r>0$ suficientemente pequeno tal que $x_{0}+r x \in W$. Daí, temos que $p(\lambda B)\left(x_{0}+r x\right) \in p(\lambda B)(W) \subseteq U \subseteq$ $M \Rightarrow p(\lambda B) x_{0}+p(\lambda B) r x \in M$. Como $p(\lambda B) x_{0} \in M$ (pois $x_{0} \in W$ e $p(\lambda B)(W) \subseteq U \subseteq M$ ), então $p(\lambda B) x_{0}+p(\lambda B) r x-p(\lambda B) x_{0}=p(\lambda B) r x \in M$. Dividindo por $r$, obtemos que $p(\lambda B) x \in M$, como desejado.

Vimos, no terceiro capítulo, o Teorema 3.9. Este teorema, apresentado e demonstrado por Le em [17], consiste em um upgrade no SHC, como discutido naquele capítulo. De maneira totalmente análoga, podemos fazer o mesmo upgrade com o Critério de Sub-Ciclicidade, culminando no próximo teorema. Vale dizer que a demonstração que daremos é totalmente adaptada e baseada naquela feita por Le em [17, Teorema 2.5]:

\footnotetext{
${ }^{29}$ Vide o Exemplo 1.18

${ }^{30}$ Observe que podemos considerar apenas $\operatorname{deg} p>0$ pois não é difícil encontrar abertos $U, V$ de $M$ tais que, para todo polinômio $p$ de grau zero, temos que $[p(\lambda B)]^{-1}(U) \cap V=\emptyset$. O motivo para querermos considerar apenas $\operatorname{deg} p>0$ é simples: vamos mostrar que se $[p(\lambda B)]^{-1}(U) \cap V$ é um aberto não-vazio de $M$, então $p(\lambda B)(M) \subseteq M, o$ que é claramente um absurdo - a não ser que tenhamos justamente $\operatorname{deg} p=0$.

${ }^{31}$ Se o grau de $p$ é $k>m$, tome o vetor $e_{k+1} \in M$. Desta forma, teremos que a primeira coordenada de $p(\lambda B) e_{k+1}$ é não-nula e, portanto, $p(\lambda B) e_{k+1} \notin M$. Agora, se $0<k \leq m$, basta tomar o vetor $e_{m+1} \in M$ : de maneira análoga, teremos que a coordenada $m-k+1$ de $p(\lambda B) e_{k+1}$ é não-nula. Como $m \geq m-k+1 \geq 1$, então é claro que $p(\lambda B) e_{k+1} \notin M$.
} 
Teorema 4.39. Sejam $X$ um espaço de Banach de dimensão infinita, $T: X \rightarrow X$ um operador linear limitado e $M$ um subespaço fechado separável de dimensão infinita de $X$ tal que $M \neq X$. Suponhamos que existem subconjuntos $X_{0}, Y_{0} \subseteq M$ tal que $Y_{0}$ é denso em $M$ e uma sequência de polinômios $\left(p_{n}\right)_{n \in \mathbb{N}} \subseteq \mathbb{K}[x]$ tais que:

(i) $p_{n}(T)(x) \rightarrow 0$, para todo $x \in X_{0}$.

(ii) para cada $y \in Y_{0}$, existe uma sequência $\left(x_{n}\right)_{n \in \mathbb{N}} \subseteq X_{0}$ tal que

$$
x_{n} \rightarrow 0 \quad \text { e } \quad p_{n}(T) x_{n} \rightarrow y
$$

(iii) $X_{0} \subseteq \cap_{n=1}^{\infty}\left[p_{n}(T)\right]^{-1}(M)$.

Então Té $M$-cíclico.

DemonstraÇão: Seja $\left(\varepsilon_{n}\right)_{n \in \mathbb{N}}$ uma sequência de números positivos tais que

$$
\lim _{n \rightarrow \infty}\left(n \varepsilon_{n}+\sum_{k=n+1}^{\infty} \varepsilon_{k}\right)=0
$$

Como $M$ é separável e $Y_{0}$ é denso em $M$, podemos supor que $Y_{0}=\left(y_{n}\right)_{n \in \mathbb{N}}$ para alguma sequência $\left(y_{n}\right)_{n \in \mathbb{N}} \subseteq Y_{0}$. Vamos construir uma sequência $\left(x_{n_{k}}\right)_{k \in \mathbb{N}} \subseteq X_{0}$ e uma subsequência $\left(p_{n_{k}}\right)_{k \in \mathbb{N}} \subseteq$ $\left(p_{n}\right)_{n \in \mathbb{N}}$ por indução.

Usando a condição (ii), tome $x_{n_{1}} \in X_{0}$ e $n_{1} \in \mathbb{N}$ tal que

$$
\left\|x_{n_{1}}\right\|+\left\|p_{n_{1}}(T) x_{n_{1}}-y_{1}\right\|<\varepsilon_{1}
$$

Para cada $j$, usando as condições (i) e (ii) escolha $n_{j} \in \mathbb{N}$ e $x_{n_{j}} \in X_{0}$ tais que

$$
\left\|x_{n_{j}}\right\|+\left\|p_{n_{j}}(T) x_{n_{i}}\right\|+\left\|p_{n_{i}}(T) x_{n_{j}}\right\|+\left\|p_{n_{j}}(T) x_{n_{j}}-y_{j}\right\|<\varepsilon_{j}
$$

para todo $i<j$.

Seja $x=\sum_{i=1}^{\infty} x_{n_{i}}$. Como $\sum_{i=1}^{\infty}\left\|x_{n_{i}}\right\| \leq \sum_{i=1}^{\infty} \varepsilon_{i}<\infty$, então $x$ está bem definido. Pela condição (iii), temos que $p_{n_{j}}(T) x \in M$, para todo $j \in \mathbb{N}$. Logo:

$$
\begin{aligned}
\left\|p_{n_{j}}(T) x-y_{j}\right\| & =\left\|p_{n_{j}}(T) x_{n_{j}}-y_{j}+\sum_{i=1}^{j-1} p_{n_{j}}(T) x_{n_{i}}+\sum_{i=j+1}^{\infty} p_{n_{j}}(T) x_{n_{i}}\right\| \\
& \leq\left\|p_{n_{j}}(T) x_{n_{j}}-y_{j}\right\|+\sum_{i=1}^{j-1}\left\|p_{n_{j}}(T) x_{n_{i}}\right\|+\sum_{i=j+1}^{\infty}\left\|p_{n_{j}}(T) x_{n_{i}}\right\| \\
& \leq j \varepsilon_{j}+\sum_{i=j+1}^{\infty} \varepsilon_{i}
\end{aligned}
$$

Temos, por hipótese, que $\lim _{j \rightarrow \infty}\left(j \varepsilon_{j}+\sum_{i=j+1}^{\infty} \varepsilon_{i}\right)=0$. Assim, como $\left(y_{n}\right)_{n \in \mathbb{N}}$ é denso em $Y_{0}$ e $Y_{0}$ é denso em $M$, a desigualdade em destaque acima nos mostra que que $\left(p_{n_{j}}(T) x\right)_{j \in \mathbb{N}} \subseteq M$ é denso em $M$. 
Com isso, segue por definição que $T$ é $M$-cíclico, como desejado. 


\section{Capítulo 5}

\section{Considerações Finais}

Apesar de termos apresentado alguns novos resultados, ao longo desta tese também vimos que existem vários problemas ainda sem resposta. Isto é natural uma vez que, como mencionamos na introdução, o conceito de sub-hiperciclicidade é bem recente. Neste último capítulo vamos recapitular alguns destes problemas, fazer algumas considerações acerca deles e apresentar outros que pensamos ser interessantes, tendo em mente que todos estes problemas também servem como motivação para futuras pesquisas.

No que segue, a numeração dos problemas apresentados será consistente com a que foi adotada previamente no texto; ou seja, se um problema abaixo já foi discutido anteriormente, então ele será apresentado com mesma numeração que foi adotada previamente, enquanto o número atribuído para um novo problema será o menor número ainda não adotado.

\section{Sub-Hiperciclicidade}

Vamos recapitular três problemas vistos no segundo capítulo:

Problema 1. Se $T$ é sub-hipercíclico para $M_{1}$ e $T^{-1}$ é sub-hipercíclico para $M_{2}$, qual a relação entre $M_{1}$ e $M_{2}$ ?

Problema 2. Se $T$ é sub-hipercíclico para $M_{1}$ e $\lambda T$ é sub-hipercíclico para $M_{2}$, qual a relação entre $M_{1}$ e $M_{2}$ ?

Problema 3. Se $T^{n}$ é sub-hipercíclico para $M_{n}$, para $n \geq 1$, qual a relação entre os $M_{n}$ ?

Tais problemas foram levantados pelos autores de [5] em 2016 e, até agora, permanecem sem solução. Vimos, no segundo capítulo, que é improvável que uma solução de tais problemas envolva um isomorfismo entre subespaços em questão. No entanto, tal isomorfismo pode aparecer em casos particulares: por exemplo, dado $n>1$, então pelo Teorema 2.20 existe um subespaço $M_{n}$ tal que $T$ é $M_{n}$-hipercíclico e $T^{n}$ é $A\left(M_{n}\right)$-hipercíclico, onde $A$ é um operador linear invertível.

Vale ressaltar ainda que o exemplo dado no parágrafo anterior contém a hipótese oculta de que $T$ é hipercíclico - assim como os problemas levantados acima. Sem essa hipótese, tais problemas tornam-se muito mais difíceis de se responder, uma vez que se $T$ não é hipercíclico - mas somente sub-hipercíclico - não é nem conhecido se as três propriedades em questão nos problemas anteriores valem. Isto nos leva ao próximo problema: 
Problema 6. Seja $\lambda \in \mathbb{K}$ tal que $|\lambda|=1$. Suponha que $T$ seja sub-hipercíclico mas não hipercíclico. Então seriam $T^{-1}, \lambda T$ e $T^{n}$ sub-hipercíclicos, para $n>1$ ?

Uma vez que as propriedades citadas no problema acima valem para operadores hipercíclicos, uma tentativa óbvia - e que fizemos uso frequentemente durante este trabalho - consiste em tentar adaptar as conhecidas demonstrações de tais propriedades para o contexto de operadores sub-hipercíclicos. No entanto, como esperado, algumas dificuldades surgem: por exemplo, tentar adaptar diretamente a demonstração do Teorema de Ansari para operadores sub-hipercíclicos não é viável, uma vez que o Teorema de Ansari faz uso do Teorema de Herrero-Bourdon (Teorema 1.33 ) - e este último teorema também não se traduz diretamente para operadores sub-hipercíclicos ${ }^{1}$

Por fim, temos mais dois problemas relativos a sub-hiperciclicidade: o já discutido Problema 4 e um novo problema que é bastante similar.

Problema 4. Dados $T, S$ hipercíclicos, existe $M$ tal que $T$ e $S$ são $M$-hipercíclicos?

Problema 7. Existe um espaço de Banach $X$ e um operador hipercíclico $T \in B(X)$ tal que $T$ é $M$-hipercíclico para todo subespaço $M \neq X$ fechado de dimensão infinita?

Para o Problema 4 tentamos adotar uma estratégia similar àquela adotada na demonstração do Teorema 2.6. feita em [5]. Nossa intenção era utilizar tal estratégia para, evidentemente, construirmos um subespaço $M$ que tornaria tanto $T$ quanto $S$ operadores $M$-hipercíclicos. No entanto, a natureza indutiva da demonstração feita em [5] não nos permite, até onde tentamos, fazer isso.

Tentamos deixar claro, ao longo deste trabalho, que a interação entre um operador hipercíclico e um subespaço no qual ele é $M$-hipercíclico ainda ocorre de uma maneira que não é totalmente compreendida. A natureza "irregular" da órbita de um operador dentro do subespaço tem sido, até agora, uma das principais dificuldades no estudo de operadores sub-hipercíclicos. Podemos atestar tal sentimento lembrando do Problema 6; as propriedades "elementares" que valem para operadores hipercíclicos ainda são desconhecidas para operadores sub-hipercíclicos. Tudo isto faz com que o Problema 7 seja, aparentemente, bastante difícil de obter uma resposta.

\section{Ciclicidade e Hiperciclicidade}

O problema que motivou todo o estudo feito no Capítulo 4 deste trabalho foi o seguinte:

Problema 5. Existe um espaço de Banach $X$ tal que todo operador hipercíclico $T$ satisfaz o Critério de Hiperciclicidade?

Vimos, na terceira seção do capítulo em questão, que adaptar diretamente a construção de Bayart e Matheron para o espaço de Argyros-Haydon não funciona. Isto nos leva a procurar outras maneiras de construir um operador (ou um espaço inteiro). Por outro lado, tal insucesso também nos permite suspeitar que o espaço de Argyros-Haydon possa ser um espaço que responda o problema acima.

\footnotetext{
${ }^{1}$ Basta lembrar, por exemplo, do Exemplo 2.2 o operador $T \oplus I$ é sub-hipercíclico em $X \oplus X$ mas, como todo vetor sub-hipercíclico de $T \oplus I$ deve ser da forma $x \oplus 0$, então é claro que o conjunto de vetores sub-hipercíclicos não pode formar um subespaço denso em $X \oplus X$.
} 
Outro fator interessante que corrobora com essa suspeita é o fato de que, como comentamos no começo do quarto capítulo, todos os exemplos conhecidos de operadores hipercíclicos da forma $\lambda I+K$ satisfazem o Critério - inclusive aquele construído no Teorema 1.27. Como operadores compactos são, de certa maneira, "parecidos" uns com os outros, somos levados a acreditar que todo operador hipercíclico da forma $\lambda I+K$ satisfaz o Critério. No entanto, tal convicção ainda não se materializou em uma demonstração, como deixamos claro ao enunciar o problema abaixo:

Problema 8. Será que todo operador hipercíclico da forma $\lambda I+K$ satisfaz o Critério de Hiperciclicidade?

Como os exemplos de De La Rosa/Read e Bayart/Matheron surgiram há mais de dez anos, então este espaço de tempo decorrido sugere que estes dois últimos problemas são de difícil resolução. Um dos motivos pelo qual se dá esta dificuldade ocorre pelo fato de que não é ainda tão claro como a compacidade interage com a hiperciclicidade (além do fato de que o operador $K$ é cíclico, como estudamos no quarto capítulo). Várias propriedades relativas a operadores hipercíclicos não se traduzem necessariamente para o operador cíclico $K$ - e ainda não é exatamente claro o porquê.

No capítulo quatro deixamos passar um pequeno detalhe. Vimos (e discutimos) a necessidade de colocar a hipótese de $X$ ser um espaço de Banach complexo na Proposição 4.13. Apesar de espaços de Banach complexos serem, em geral, os mais estudados, não é claro se a mesma proposição ainda vale para espaços de Banach reais:

Problema 9. Se $X$ é um espaço de Banach real e um operador $T \in \mathcal{B}(X)$ é tal que $T \oplus T$ é cíclico, então $T$ satisfaz o Critério de Ciclicidade?

A óbvia tentativa de complexificação do espaço não funciona neste caso (nem no caso de hiperciclicidade), ao contrário de outras propriedades de dinâmica linear (ver, por exemplo, [14, Corolário 2.51, p.51]). No entanto, operadores hipercíclicos são tais que $p(T)$ tem imagem densa, para todo polinômio $p \in \mathbb{R}[x]$ não-nulo, o que torna possível a conclusão desejada para operadores hipercíclicos, como discutimos no quarto capítulo.

\section{Sub-Ciclicidade}

A primeira grande questão que surgiu quando definimos sub-ciclicidade foi a seguinte: deveríamos adotar $A:=[\operatorname{span} \operatorname{orb}(x, T)] \cap M$ ou $B:=\operatorname{span}[\operatorname{orb}(x, T) \cap M]$ como o conjunto de interesse na definição do conceito de sub-ciclicidade? Vimos que era coerente adotar o primeiro conjunto por vários motivos; em particular, vimos que, em geral, $B \subseteq A$ e $A \nsubseteq B$, como mostramos no Exemplo 4.26 .

No entanto, em tal exemplo o próprio conjunto $A$ não é denso em $M$, o que leva a pensar: será que nos casos realmente importantes existe mesmo alguma diferença entre os conjuntos $A$ e $B$ ? Queremos dizer o seguinte: se o conjunto $A$ for denso em $M$ (ou seja, o operador em questão for $M$-cíclico, segundo nossa definição), será que o conjunto $B$ também não será denso em $M$ - e, portanto, toda essa discussão feita entorno da escolha do conjunto é desnecessária? Destacamos tal questionamento a seguir: 
Problema 10. Sejam $X$ um espaço de Banach e $M \neq X$ um subespaço separável de dimensão infinta. Se $T \in \mathcal{B}(X)$ é tal que $[\operatorname{span}$ orb $(x, T)] \cap M$ é denso em $M$, para algum $x \in X$, então vale que $\operatorname{span}[\operatorname{orb}(x, T) \cap M]$ é denso em $M$ ? Ou que $\operatorname{span}[\operatorname{orb}(y, T) \cap M]$ seja denso em $M$, para algum $y \in X$ ? 


\section{Bibliografia}

[1] Ahlfors, L., Complex Analysis. Coleção International Series in Pure and Applied in Mathematics. McGraw-Hill: New York, 1979.

[2] Albiac, F., e Kalton, N.J., Topics in Banach Space Theory. Coleção Graduate Texts in Mathematics, 233. Springer-Verlag: New York, 2016.

[3] Argyros, S. e Haydon, R., "A Hereditarily Indecomposable $\mathcal{L}_{\infty}$-space that Solves the ScalarPlus-Compact Problem", Acta Math. 206 (2011), 1-54.

[4] Argyros, S. e Motakis, P., "The Scalar-Plus-Compact Property in Spaces without Reflexive Subspaces", Trans. Amer. Math. Soc. 371 (2019), no. 3, 1887-1924.

[5] Bamerni, N., Kadets, V. e Kiliçman A., "Hypercyclic Operators Are Subspace Hypercyclic", J. Math. Anal. Appl. 435 (2016), 1812-1815.

[6] Bayart, F. e Matheron É., Dynamics of Linear Operators. Coleção Cambridge Tracts in Mathematics, 179. Cambridge: New York, 2009.

[7] Bayart, F. e Matheron É., "Hypercyclic Operators Failing the Hypercyclic Criterion on Classical Banach Spaces", J. Funct. Anal. 250 (2007), 426-441.

[8] Bès, J. e Peris, A., "Hereditarily Hypercyclic Operators", J. Funct. Anal. 167 (1999), 94-112.

[9] Chalendar, I. e Partington, J., "An Image Problem for Compact Operators", Proc. Amer. Math. Soc. 134 (2006), 1391-1396.

[10] De La Rosa, M. e Read, C.J., "A Hypercyclic Operator whose Direct Sum $T \oplus T$ is not Hypercyclic", J. Operator Theory 61 (2009), no. 2, 369-380.

[11] Duren, P. e Schuster, A., Bergman Spaces. Coleção Mathematical Surveys and Monographs, 100. AMS: Rhode Island, 2004.

[12] Godefroy, G. e Shapiro, J.H., "Operators with Dense, Invariant, Cyclic Vector Manifolds", J. Funct. Anal. 98 (1991), 229-269.

[13] Grivaux, S., "Hypercyclic Operators, Mixing Operators, and the Bounded Steps Problem", J. Funct. Anal. 202 (2003), 486-503.

[14] Grosse-Erdmann, K. e Peris, A., Linear Chaos. Coleção Universitext. Springer: Londres, 2011. 
[15] Halmos, P., A Hilbert Space Problem Book. Coleção Graduate Texts in Mathematics, 19. Springer-Verlag: New York, 1982.

[16] Jiménez-Munguía, R., Martínez-Avendaño, R., e Peris, A., "Some Questions About Subspace-Hypercyclic Operators", J. Math. Anal. Appl. 408 (2013), 209-212.

[17] Le, Can M., "On Subspace-Hypercyclic Operators", Proceedings of the AMS 139 (2011), no. 8, 2847-2852.

[18] Madore, B. e Martínez-Avendaño, R., "Subspace Hypercyclicity", J. Math. Anal. Appl. 373 (2011), 502-511.

[19] Manoussakis, A., Pelczar-Barwacz, A. e Swietek, M., "An Unconditionally Saturated Banach Space with the Scalar-Plus-Compact Property", J. Funct. Anal. 272 (2017), 4944-4983.

[20] Martínez-Avendaño, R. e Rosenthal, P., An Introduction to Operators on the HardyHilbert Space. Coleção Graduate Texts in Mathematics, 237. Springer-Verlag: New York, 2007.

[21] Martínez-Avendaño, R. e Zatarain-Vera, O., "Subspace Hypercyclicity for Toeplitz Operators", J. Math. Anal. Appl. 422 (2015), 772-775.

[22] Megginson, R.E., An Introduction to Banach Space Theory. Coleção Graduate Texts in Mathematics, 183. Springer-Verlag: New York, 1998.

[23] Ovsepian, R. e PeŁczyński, A., "On the Existence of a Fundamental Total and Bounded Biorthogonal Sequence in Every Separable Banach Space, and Related Constructions of Uniformly Bounded Orthonormal Systems in L"," Studia Math. 54 (1975), 149-159.

[24] Rezaei, H., "On Operators with Orbits Dense Relative to Nontrivial Subspaces", Funct. Anal. Appl. 51 (2017), 112-122.

[25] Rolewicz, S., "On Orbits of Elements", Studia Math. 32 (1969), 17-22.

[26] Subrahmonian Moothathu, T.K., "Hypercyclic Orbits Intersect Subspaces in Wild Ways", J. Math. Anal. Appl. 446 (2017), 1183-1195. 